\title{
QUANTUM LIOUVILLE THEORY IN THE BACKGROUND FIELD FORMALISM I. COMPACT RIEMANN SURFACES
}

\author{
LEON A. TAKHTAJAN AND LEE-PENG TEO
}

\begin{abstract}
Using Polyakov's functional integral approach and the Liouville action functional defined in [ZT87c and TT03a, we formulate quantum Liouville theory on a compact Riemann surface $X$ of genus $g>1$. For the partition function $\langle X\rangle$ and correlation functions with the stress-energy tensor components $\left\langle\prod_{i=1}^{n} T\left(z_{i}\right) \prod_{k=1}^{l} \bar{T}\left(\bar{w}_{k}\right) X\right\rangle$, we describe Feynman rules in the background field formalism by expanding corresponding functional integrals around a classical solution, the hyperbolic metric on $X$. Extending analysis in Tak93, Tak94, Tak96a, Tak96b, we define the regularization scheme for any choice of the global coordinate on $X$. For the Schottky and quasi-Fuchsian global coordinates, we rigorously prove that one- and two-point correlation functions satisfy conformal Ward identities in all orders of the perturbation theory. Obtained results are interpreted in terms of complex geometry of the projective line bundle $\mathscr{E}_{c}=\lambda_{H}^{c / 2}$ over the moduli space $\mathfrak{M}_{g}$, where $c$ is the central charge and $\lambda_{H}$ is the Hodge line bundle, and provide the Friedan-Shenker [FS87] complex geometry approach to CFT with the first non-trivial example besides rational models.
\end{abstract}

\section{Contents}

1. Introduction

2. Classical Liouville theory

2.1. Liouville action functional 10

2.2. The stress-energy tensor $\quad 12$

3. Quantum Liouville theory 14

3.1. Feynman rules for the partition function $\quad 15$

\begin{tabular}{lll}
3.2. & Feynman rules for correlation functions & 19 \\
\hline
\end{tabular}

4. Deformation theory 22

4.1. Schottky and Teichmüller spaces $\quad 22$

4.2. Formal geometry on deformation spaces 24

4.3. Variational formulas 25

5. The propagator 28

5.1. Behavior near diagonal and explicit formulas 30

5.2. Variational formulas 34

6. One-point correlation functions $\quad 40$

6.1. Classical contribution 40

6.2. One-loop contribution 41

6.3. Higher loops 42 
7. Two-point correlation function - TT equation 46

7.1. Classical contribution 47

7.2. One-loop contribution 48

7.3. Higher loops 52

8. Two-point correlation function $-T \bar{T}$ equation

8.1. Classical contribution 54

8.2. One-loop contribution 5

8.3. Higher loops 57

9. Conformal Ward identities and modular geometry 58

Appendix A. Belavin-Knizhnik theorem and the $T \bar{T}$ equation 61

Appendix B. The stress-energy tensor and the action functional 64

References 65

\section{INTRODUCTION}

Classical Liouville theory is a Euclidean field theory associated with hyperbolic Riemann surfaces. Complete conformal metrics $d s^{2}$ on a Riemann surface $X$ are classical fields of the theory, and the so-called Liouville equation - the equation $K\left(d s^{2}\right)=-1$, where $K\left(d s^{2}\right)$ is a Gaussian curvature, is the corresponding Euler-Lagrange equation. According to the uniformization theorem, it has a unique solution - the hyperbolic metric on $X$. The quantized Liouville theory describes "quantum corrections" to hyperbolic geometry of $X$ by taking into account fluctuations around the hyperbolic metric. In 1981, Polyakov formulated a functional integral approach to bosonic string theory, and made a fundamental discovery that quantum Liouville theory is a conformal anomaly for non-critical strings [Pol81]. Thus in order to find correlation functions of vertex operators of the bosonic string in any dimension $D$ (and not only for $D=26$ ), one needs to know correlation functions of the Liouville vertex operators $V_{\alpha}(z)=e^{\alpha \varphi(z)}$, where $d s^{2}=e^{\varphi(z)}|d z|^{2}$ is the Liouville field - a conformal metric on $X$. The fundamental property that classical fields and equation of motion of the Liouville theory are conformally invariant, led Belavin, Polyakov and Zamolodchikov to their formulation of the two-dimensional Conformal Field Theory (CFT) [BPZ84]. Though the problem of computing correlation functions of the Liouville vertex operators, needed for non-critical string theory, is still outstanding, in the works of Dorn and Otto [DO94, and of Zamolodchikov and Zamolodchikov [ZZ96] the quantum Liouville theory was formulated as a non-rational model of CFT with a continuous spectrum of conformal dimensions (see the review [Tes01] for a complete account and references).

In [Pol82, Polyakov proposed a functional integral representation for correlation functions of the Liouville vertex operators in the form needed for the non-critical string theory. This so-called geometric approach to the quantum Liouville theory was formalized and developed in [Tak93, Tak94, 
Tak96a, Tak96b. In this formulation, correlation functions of Liouville vertex operators on the Riemann sphere $\mathbb{P}^{1}$ are defined by

$$
\left\langle V_{\alpha_{1}}\left(z_{1}\right) \ldots V_{\alpha_{n}}\left(z_{n}\right)\right\rangle=\int_{\mathscr{C} \mathscr{M}_{\alpha}\left(\mathbb{P}^{1}\right)} e^{-\frac{1}{2 \pi \hbar} S_{\alpha}(\varphi)} \mathscr{D} \varphi,
$$

where $\hbar>0$ plays the role of Planck's constant, $\mathscr{C} \mathscr{M}_{\alpha}\left(\mathbb{P}^{1}\right)$ is the space of all smooth conformal metrics $e^{\varphi(z)}|d z|^{2}$ on $\mathbb{P}^{1} \backslash\left\{z_{1}, \ldots, z_{n}\right\}$ which have conical singularities at the insertion points

$$
e^{\varphi(z)} \simeq \frac{1}{\left|z-z_{i}\right|^{2 \hbar \alpha_{i}}} \quad \text { as } \quad z \rightarrow z_{i}, \quad i=1, \ldots, n,
$$

and $S_{\alpha}(\varphi)$ is the Liouville action functional defined in Tak96b]. Here $\hbar \alpha_{i} \leq$ 1 and $\sum_{i=1}^{n} \hbar \alpha_{i}>2$. When $\hbar \alpha_{i}=1$, which corresponds to the puncture vertex operator, asymptotic (1.2) is replaced by $e^{\varphi(z)} \simeq\left|z-z_{i}\right|^{-2}\left(\log \left|z-z_{i}\right|\right)^{-2}$. According to BPZ84, conformal symmetry of the theory manifests itself through conformal Ward identities for correlation functions with insertions of components of the stress-energy tensor. The Ward identity for the $(2,0)$ component $T(\varphi)=\frac{1}{\hbar}\left(\varphi_{z z}-\frac{1}{2} \varphi_{z}^{2}\right)$ has the form

$$
\left\langle T(z) X_{\alpha}\right\rangle=\sum_{i=1}^{n}\left(\frac{\Delta_{\alpha_{i}}}{\left(z-z_{i}\right)^{2}}+\frac{\partial_{z_{i}}}{z-z_{i}}\right)\left\langle X_{\alpha}\right\rangle,
$$

where $X_{\alpha}=V_{\alpha_{1}}\left(z_{1}\right) \ldots V_{\alpha_{n}}\left(z_{n}\right)$,

$$
\left\langle T(z) X_{\alpha}\right\rangle=\int_{\mathscr{C} \mathscr{M}_{\alpha}\left(\mathbb{P}^{1}\right)} T(\varphi)(z) e^{-\frac{1}{2 \pi \hbar} S_{\alpha}(\varphi)} \mathscr{D} \varphi,
$$

and $\Delta_{\alpha_{i}}=\alpha_{i}\left(2-\hbar \alpha_{i}\right) / 2$ are conformal dimensions of the vertex operators $V_{\alpha_{i}}$. Note that since $\left\langle X_{\alpha}\right\rangle$ and $\left\langle T(z) X_{\alpha}\right\rangle$ have been already defined by functional integrals (1.1) and (1.4), the Ward identity (1.3) actually requires a proof. BPZ conformal Ward identities were generalized to higher genus Riemann surfaces in [EO87].

At the classical level equation (1.3) (and a similar equation for compact Riemann surfaces) represents a non-trivial relation between the accessory parameters of the Fuchsian uniformization of the Riemann surface $X=$ $\mathbb{P}^{1} \backslash\left\{z_{1}, \ldots, z_{n}\right\}$ and the classical Liouville action - the critical value of the Liouville action functional. It was proved in [ZT87b] (and in [ZT87c for the compact case; see also the discussion in Tak89, Tak92]).

The background field formalism for puncture operators - a perturbative expansion in $\hbar$ around the classical solution for the partition function and correlation functions with insertions of the stress-energy tensor, was developed in Tak93, Tak94]. The results, summarized in Tak96a], are the following.

\footnotetext{
${ }^{1}$ This should be compared with the standard CFT approach to quantum Liouville theory, where Ward identities are built into the construction of the Hilbert space of states which carries a representation of the Virasoro algebra.
} 
- Rigorous definition of $\left\langle X_{\alpha}\right\rangle$ and $\left\langle\prod_{i=1}^{n} T\left(z_{i}\right) \prod_{k=1}^{l} \bar{T}\left(\bar{w}_{k}\right) X_{\alpha}\right\rangle$ in all orders of the perturbation theory, and the proof of the conformal Ward identity (1.3) at the one-loop level. The latter follows from the formula for the first variation of the Selberg zeta function $Z(s)$ at $s=2$ in TZ91.

- The proof of conformal Ward identities with two insertions of the stress-energy tensor at the classical level, based on results in ZT87b, ZT87c. Equivalence between the Ward identity for $\left\langle T(z) \bar{T}(\bar{w}) X_{\alpha}\right\rangle$ at the one-loop level, and the local index theorem for families of $\bar{\partial}$-operators on punctured Riemann surfaces, proved in [TZ91].

- The asymptotic

$$
\left\langle T(z) T(w) X_{\alpha}\right\rangle=\frac{c / 2}{(z-w)^{4}}+O\left(|z-w|^{-2}\right) \quad \text { as } \quad w \rightarrow z
$$

valid in all orders of the perturbation theory, where

$$
c=\frac{12}{\hbar}+1
$$

is the central charge of quantum Liouville theory, given by the sum of classical contribution $\frac{12}{\hbar}$ and one-loop correction 1 .

The present paper is a long overdue sequel to [Tak96a]. We extend the results in Tak96a to all orders of the perturbation theory, with precise formulations and complete proofs. To emphasize the invariant geometric meaning of our results, and avoid any additional analytic ramifications due to non-compactness, we concentrate on the case of compact Riemann surfaces. The important case of the Riemann surfaces with punctures will be considered elsewhere.

Namely, let $X$ be a compact Riemann surface of genus $g>1$. We define the partition function $\langle X\rangle$ as the following functional integral

$$
\langle X\rangle=\int_{\mathscr{C} \mathscr{M}(X)} e^{-\frac{1}{2 \pi \hbar} S(\varphi)} \mathscr{D} \varphi,
$$

where $\mathscr{C} \mathscr{M}(X)$ is the set of all smooth conformal metrics on $X$, and $S$ is the Liouville action functional. It is known TT03a that the definition of $S$ depends on the choice of a global coordinate on $X$ - a representation $X \simeq \Gamma \backslash \Omega$, where $\Gamma$ is a Kleinian group with an invariant component $\Omega$. For our purposes it is sufficient to consider the case when $\Gamma$ is either a Schottky group, or a quasi-Fuchsian group. Corresponding action functionals were defined in [TR7c and [TT03a] respectively.

A comparison with (1.1) shows that $\langle X\rangle$ can be interpreted as a "correlation function of handle operators". Ultimately, for every $\hbar>0$ we would like to define $\langle X\rangle$ as a function on the corresponding Schottky space $\mathfrak{S}_{g}$, or Teichmüller space $\mathfrak{T}_{g}$, which parameterizes marked Riemann surfaces of genus $g>1$. However, we can only define $\langle X\rangle$ perturbatively as a "formal function" - a formal power series in $\hbar$ with coefficients being smooth functions 
on $\mathfrak{S}_{g}$ or $\mathfrak{T}_{g}$. This is done in the background field formalism by considering the perturbation expansion of the functional integral (1.5) around a classical solution. Corresponding UV-divergencies, following [Tak93, are regularized by using a reparametrization-invariant definition of the propagator at coincident point:2. In this regularization scheme only classical contribution to the partition function $\langle X\rangle$ - a term of order $\hbar^{-1}$ - depends on the choice of a global coordinate on $X$. All other terms in the formal Taylor series expansion of $\langle X\rangle$ are well-defined functions on the moduli space $\mathfrak{M}_{g}$ of compact Riemann surfaces of genus $g>1$.

Multi-point correlation functions $\left\langle\prod_{i=1}^{n} T\left(z_{i}\right) \prod_{k=1}^{l} \bar{T}\left(\bar{w}_{k}\right) X\right\rangle$ with insertions of the stress-energy tensor are defined in a similar way. The UVdivergence arising from a tadpole graph is regularized as in Tak93, Tak96a, whereas divergencies arising from graphs with self-loops are regularized in a way similar to the regularization of $\langle X\rangle$. In this definition, only classical and one-loop contributions to one-point correlation functions depend nontrivially on the choice of a global coordinate on $X$. All other higher loop contributions to $\langle T(z) X\rangle$ and $\langle\bar{T}(\bar{z}) X\rangle$ are correspondingly $(2,0)$ and $(0,2)$ tensors on $X$. Similarly, all terms in the irreducible multi-point correlation functions $\left\langle\left\langle\prod_{i=1}^{n} T\left(z_{i}\right) \prod_{k=1}^{l} \bar{T}\left(\bar{w}_{k}\right) X\right\rangle\right\rangle$ with $n+l \geq 2$ are tensors of type $(2,0)$ and $(0,2)$ on $X$ in $z_{1}, \ldots, z_{n}$ and $w_{1}, \ldots, w_{l}$, which are symmetric with respect to these two groups of variables.

Our main results are given in Theorems 6.1, 7.1 and 8.1. Succinctly, partition and correlation functions of the quantum Liouville theory, defined in Section 3, satisfy the conformal Ward identities in all orders of the perturbation theory. As a corollary, the quantum Liouville theory in the background field formalism is a conformal field theory with the central charge $c=\frac{12}{\hbar}+1$.

To present the first result (we refer to Theorem 6.1 for the invariant geometric formulation), let $X \simeq \Gamma \backslash \Omega$ be a Riemann surface of genus $g>1$ with a Schottky global coordinate, $\mu$ be a harmonic Beltrami differential for $\Gamma$, and let $X^{\varepsilon \mu} \simeq \Gamma^{\varepsilon \mu} \backslash \Omega^{\varepsilon \mu}$ be the corresponding holomorphic family of Riemann surfaces (see Section 4). Then

$$
\left.\frac{\partial}{\partial \varepsilon}\right|_{\varepsilon=0} \log \left\langle X^{\varepsilon \mu}\right\rangle=-\frac{1}{\pi} \iint_{F}\left(\langle\langle T(z) X\rangle\rangle-\frac{1}{12} \mathcal{S}\left(J^{-1}\right)(z)\right) \mu(z) d^{2} z,
$$

where $F$ is a fundamental domain for $\Gamma$ in $\Omega, J: \mathbb{U} \rightarrow \Omega$ is the covering map of $\Omega$ by the upper half-plane $\mathbb{U}$, and $\mathcal{S}(f)$ stands for the Schwarzian derivative of a holomorphic function $f$. Equation (1.6) is a precise analog of the BPZ conformal Ward identity (1.3) for a compact Riemann surface $X$. We emphasize that both sides of (1.6) are defined by corresponding functional integrals, and the equation is valid in all orders of the perturbation expansion.

To state the second result (see Theorem 7.1 for the invariant formulation), let $G(z, w)$ be the propagator of the quantum Liouville theory - the kernel

\footnotetext{
${ }^{2}$ In the non-compact case one should also regularize IR-divergencies at the punctures.
} 
of a resolvent operator $\frac{1}{2}\left(\Delta_{0}+\frac{1}{2}\right)^{-1}$, where $\Delta_{0}$ is the Laplace operator of the hyperbolic metric $d s^{2}=e^{\varphi_{c l}(z)}|d z|^{2}$ on $X$, acting on functions, and let $R(z, w)=4 e^{-\varphi_{c l}(z)} \partial_{\bar{z}} \mathcal{D}_{w} G(z, w)$, where $\mathcal{D}_{w}=\partial_{w}^{2}-\left(\partial_{w} \varphi_{c l}\right)(w) \partial_{w}$ (see Section (5). Then in all orders of the perturbation expansion,

$$
\begin{gathered}
\left.\frac{\partial^{2}}{\partial \varepsilon_{1} \partial \varepsilon_{2}}\right|_{\varepsilon_{1}=\varepsilon_{2}=0} \log \left\langle X^{\varepsilon_{1} \mu+\varepsilon_{2} \nu}\right\rangle \\
=\frac{1}{\pi^{2}} \iint_{F} \iint_{F}\left(\langle\langle T(z) T(w) X\rangle\rangle-\frac{6}{\hbar} K(z, w)-\frac{\pi}{6} \mathcal{D}_{z} \mathcal{D}_{w} G(z, w)\right. \\
\left.-\pi\left(2 \partial_{z} R(z, w)+R(z, w) \partial_{z}\right)\left(\langle\langle T(z) X\rangle\rangle-\frac{1}{12} \mathcal{S}\left(J^{-1}\right)(z)\right)\right) \mu(z) \nu(w) d^{2} z d^{2} w \\
=\frac{1}{\pi^{2}} \iint_{F} \iint_{F}\left(\langle\langle T(z) T(w) X\rangle\rangle-\frac{6}{\hbar} K(z, w)-\frac{\pi}{6} \mathcal{D}_{z} \mathcal{D}_{w} G(z, w)\right) \mu(z) \nu(w) d^{2} z d^{2} w .
\end{gathered}
$$

Here $\mu, \nu$ are harmonic Beltrami differentials for $\Gamma$, and

$$
K(z, w)=\sum_{\gamma \in \Gamma} \frac{\gamma^{\prime}(w)^{2}}{(z-\gamma w)^{4}} .
$$

It follows from (1.7) that $\langle\langle T(z) T(w) X\rangle\rangle$ is a meromorphic quadratic differential for $\Gamma$ in $z$ and $w$, with the only fourth order pole at $z=w$, and that for $w \rightarrow z$

$$
\begin{aligned}
\langle\langle T(z) T(w) X\rangle\rangle= & \frac{c / 2}{(z-w)^{4}}+\left(\frac{2}{(z-w)^{2}}-\frac{\partial_{z}}{z-w}\right)\langle\langle T(z) X\rangle\rangle \\
& + \text { regular terms as } w \rightarrow z,
\end{aligned}
$$

where $c=\frac{12}{\hbar}+1$. Equation (1.7) is a precise analog of the BPZ conformal Ward identity with two insertions of the $(2,0)$ component of the stressenergy tensor [BPZ84]. This proves that the quantum Liouville theory in the background field formalism is a CFT model with the central charge $c$.

Finally, the third result (see Theorem 8.1 and Corollary 8.2 for invariant formulations) can be stated as

$$
\begin{gathered}
\left.\frac{\partial^{2}}{\partial \varepsilon_{1} \partial \bar{\varepsilon}_{2}}\right|_{\varepsilon_{1}=\varepsilon_{2}=0} \log \left\langle X^{\varepsilon_{1} \mu+\varepsilon_{2} \nu}\right\rangle \\
=\frac{1}{\pi^{2}} \iint_{F} \iint_{F}\left(\langle\langle T(z) \bar{T}(\bar{w}) X\rangle\rangle-\frac{\pi}{6} \mathcal{D}_{z} \mathcal{D}_{\bar{w}} G(z, w)\right) \mu(z) \overline{\nu(w)} d^{2} z d^{2} w .
\end{gathered}
$$

Equation (1.9), which is valid in all orders of the perturbation expansion, is a conformal Ward identity with single insertions of $(2,0)$ and $(0,2)$ components of the stress-energy tensor (the case not considered in BPZ84). In particular, $\langle\langle T(z) \bar{T}(\bar{w}) X\rangle\rangle$ is a holomorphic $(2,0)$ tensor on $X$ in variable $z$ and anti-holomorphic $(0,2)$ tensor in variable $w$. The classical contribution to $\langle\langle T(z) \bar{T}(\bar{w}) X\rangle\rangle$ is a multiple of the Weil-Petersson metric on $\mathfrak{M}_{g}$, and (1.9) at the classical level states that the classical Liouville action is its Kähler potential [ZT87c]. At the one-loop level, as we show in Appendix 
A, (1.9) is another way of presenting the Belavin-Knizhnik theorem [BK86]. Finally, in Remark 9.1 we explain the sense in which the two-point correlation function $\langle\langle T(z) \bar{T}(\bar{w}) X\rangle\rangle$ defines a family of Kähler metrics on $\mathfrak{M}_{g}$ with Kähler potential $\pi^{2}\left(\log \langle X\rangle+\frac{\hbar}{12} \log \langle X\rangle_{c l}\right)$.

For the reader's convenience, we make the paper relatively self-contained by presenting the background material necessary for the computations. To keep the length of the paper under control, we refer to [ZT87c, TT03a for the construction of the Liouville action functional, and to [Tak92, Tak93, Tak94, Tak96a, Tak96b] for the history of the geometric approach and discussion of conformal Ward identities at classical and one-loop levels.

Here is a more detailed content of the paper. Section 2 is devoted to the classical Liouville theory. We briefly discuss Schottky, Fuchsian and quasiFuchsian uniformizations of the compact Riemann surfaces, introduce the Liouville action functional and the stress-energy tensor, and describe their main properties. In Section 3, we formulate the quantum Liouville theory in the background field formalism. Specifically, in Section 3.1 we describe the Feynman rules and the regularization scheme for the perturbative expansion of the partition function $\langle X\rangle$, and in Section 3.2 we describe the Feynman rules and the regularization scheme for multi-point correlation functions with insertions of the stress-energy tensor.

Section 4 is a "crash course" on deformation theory of compact Riemann surfaces. In Section 4.1 we define the deformation space $\mathfrak{D}(\Gamma)$, where $\Gamma$ is either a Schottky or a quasi-Fuchsian group, and describe a complex manifold structure on $\mathfrak{D}(\Gamma)$. The Schottky space $\mathfrak{S}_{g}$ is a deformation space $\mathfrak{D}(\Gamma)$ where $\Gamma$ is a Schottky group, and the Teichmüller space $\mathfrak{T}_{g}$ is a complexanalytic submanifold of $\mathfrak{D}(\Gamma)$ where $\Gamma$ is a Fuchsian group. In Section 4.2 we define the formal function on $\mathfrak{D}(\Gamma)$ as a formal power series in $\hbar$ whose coefficients are smooth functions on $\mathfrak{D}(\Gamma)$, and show that the partition function $\langle X\rangle$ and the free energy $\mathcal{F}_{X}=-\log \langle X\rangle$ give rise to formal functions on $\mathfrak{S}_{g}$ and $\mathfrak{T}_{g}$. In Section 4.3 we collect necessary variational formulas, from classical results of Ahlfors Ahl61 and Wolpert Wol86 to the formulas in ZT87c and TT03a. To the reader without prior knowledge of the deformation theory we recommend classical works [Ahl87, Ber70, Ahl61, briefly summarized in TT03a.

Section 5, where we study the propagator $G(P, Q)$ of the quantum Liouville theory, is crucial for our approach. The propagator $G(P, Q)$ is defined as the integral kernel of the resolvent operator $\frac{1}{2}\left(\Delta_{0}+\frac{1}{2}\right)^{-1}$, where $\Delta_{0}$ is the Laplace operator of the hyperbolic metric on $X$ acting on functions. In Section [5.1, using the Fuchsian global coordinate $z$ on $X \simeq \Gamma \backslash \mathbb{U}$, we represent $G(z, w)$ as the average over a Fuchsian group $\Gamma$ of the propagator $\mathcal{G}(z, w)$ on the upper half-plane $\mathbb{U}$ (method of images), which is given by an explicit formula. We determine the short-distance behavior of $G(z, w)$, and define a regularization of $G(z, w)$ and of $\partial_{z} \partial_{w} G(z, w)$ at $w=z$ by subtracting corresponding contributions of the identity element of $\Gamma$. Thus defined $G(z, z)$ gives rise to a smooth function on $X$, whereas the corresponding 
$H(z)=\partial_{z} \partial_{z} G(z, z)$ is a smooth quadratic differential for $\Gamma$, which behaves like " $1 / 12 \pi$ of the projective connection" under the changes of global coordinates. We present an explicit formula $P(z, w)=4 \mathcal{D}_{z} \mathcal{D}_{\bar{w}} G(z, w)$ for the integral kernel of the projection operator $P$ onto the subspace of holomorphic quadratic differentials on $X$. Though just being another form of the Ahlfors classical result, it plays a fundamental role in the computations in Sections 68. In Section 5.2 we prove variational formulas for the propagator and its derivatives, collected in Lemmas 5.4 and 5.6.

In Section 6, we prove the conformal Ward identity with single insertion of the stress-energy tensor — Theorem 6.1, by computing $\partial \log \langle X\rangle$ in all orders of the perturbative expansion. As we have already mentioned, at the classical level the corresponding result was proved in [ZT87c and [TT03a]. In Section 6.2, we compute $\partial \log \langle X\rangle$ at the one-loop level, and show that the result coincides with the representation of $\langle\langle T(z) X\rangle\rangle_{1-\text { loop }}$ as a sum of Feynman graphs. The computation uses the formula for $\partial \log Z(2)$ from [TZ91, Section 3], the explicit form of the kernel $P(z, w)$, and the Stokes' theorem. For higher loop terms, the statement of Theorem 6.1 is valid "graph by graph". The actual computation splits into three cases, analyzed in Section 6.3 by repeated use of the Stokes' theorem and careful analysis of boundary contributions.

In Sections 7 and 8 we prove Theorems 7.1 and 8.1 - conformal Ward identities with two insertions of the stress-energy tensor, which express the two-point correlation functions $\langle\langle T(z) T(w) X\rangle\rangle$ and $\langle\langle T(z) \bar{T}(\bar{w}) X\rangle\rangle$ in terms of the one-point correlation functions $\langle\langle T(z) X\rangle\rangle$ and $\langle\langle\bar{T}(\bar{z}) X\rangle\rangle$. By Theorem 6.1, the correlation function $\langle\langle T(z) X\rangle\rangle$ is a holomorphic quadratic differential for $\Gamma$ which corresponds to an exact $(1,0)$-form on the Schottky space $\mathfrak{S}_{g}$, and Theorem 8.1 states that the $(1,1)$-form $\bar{\partial}\langle\langle T(z) X\rangle\rangle$ on $\mathfrak{S}_{g}$ corresponds to $-\frac{1}{\pi}\langle\langle T(z) \bar{T}(\bar{w}) X\rangle\rangle$, which is a holomorphic quadratic differential for $\Gamma$ in variable $z$ and an anti-holomorphic quadratic differential for $\Gamma$ in variable $w$. On the other hand, the two-point correlation function $\langle\langle T(z) T(w) X\rangle\rangle$ is symmetric in $z$ and $w$, so it can not be represented by a $(2,0)$-form on $\mathfrak{S}_{g}$. Theorem 7.1 expresses $\langle\langle T(z) T(w) X\rangle\rangle$ as an application of a "symmetrized $(1,0)$-differential" $\partial_{s}$ to $\langle\langle T(z) X\rangle\rangle$. It is defined in Section 4.3 as follows: if $\theta=\sum_{i=1}^{d} a_{i} d t_{i}$ is a $(1,0)$-form on $\mathfrak{S}_{g}$, then $\partial_{s} \theta=\sum_{i, j=1}^{d} \frac{\partial a_{j}}{\partial t_{i}} d t_{i} \otimes_{s} d t_{j}$, where $\otimes_{s}$ stands for the symmetrized tensor product. This explains why Theorem 7.1, which is a statement about second partial derivatives of a certain formal function on $\mathfrak{S}_{g}$ rather than a statement about differential forms, looks complicated when compared with Theorem 8.1. The actual proof of Theorems 7.1 and 8.1 is based on the computation of $\partial\langle\langle T(z) X\rangle\rangle$ and $\bar{\partial}\langle\langle T(z) X\rangle\rangle$ in all orders of the perturbative expansion. Again, at the classical level, the corresponding result was proved in [ZT87c] and [TT03a], and the major computation is at the oneloop level. It is based on Theorem 6.1, variational formulas in Section 5.2, 
the explicit formula for the kernel $P(z, w)$, and repeated application of the Stokes' theorem.

In Section 9 we show that Theorem 7.1 at $w \rightarrow z$ agrees precisely with the BPZ conformal Ward identity with two insertions of the $(2,0)$ component of the stress-energy tensor, where $c=\frac{12}{\hbar}+1$. This proves that the quantum Liouville theory in the background field formalism is conformal with the central charge $c$. Using one result of Zograf [Zog89, we interpret Theorems 6.1, 7.1 and 8.1 in terms of complex geometry of the projective line bundle $\mathscr{E}_{c}=\lambda_{H}^{c / 2}$ over the moduli space $\mathfrak{M}_{g}$, where $\lambda_{H}$ is the Hodge line bundle. This agrees with (and clarifies) the Friedan-Shenker "modular geometry" approach to conformal field theory [FS87.

We conclude the paper with two appendices. In Appendix A, we show that the one-loop contribution to Theorem 8.1 gives the Belavin-Knizhnik theorem [BK86] for the case of Laplace operators acting on quadratic differentials on Riemann surfaces. In Appendix B we show how to obtain the stress-energy tensor from the Liouville action functional for the Schottky global coordinate. The corresponding result - Lemma B.1 - follows from the proof of Theorem 1 in [ZT87c].

Acknowledgments. The first author was partially supported by the NSF grant DMS-0204628.

\section{Classical Liouville theory}

Let $X$ be a compact Riemann surface of genus $g>1$, and let $\left\{U_{\alpha}\right\}_{\alpha \in A}$ be a complex-analytic atlas on $X$ with charts $U_{\alpha}$, local coordinates $z_{\alpha}$ : $U_{\alpha} \rightarrow \mathbb{C}$, and transition functions $f_{\alpha \beta}: z_{\beta}\left(U_{\alpha} \cap U_{\beta}\right) \rightarrow z_{\alpha}\left(U_{\alpha} \cap U_{\beta}\right)$. Denote by $\mathscr{C} \mathscr{M}(X)$ the space (actually a cone) of smooth conformal metrics on $X$. Every metric $d s^{2} \in \mathscr{C} \mathscr{M}(X)$ is a collection $\left\{e^{\varphi_{\alpha}}\left|d z_{\alpha}\right|^{2}\right\}_{\alpha \in A}$, where the functions $\varphi_{\alpha} \in C^{\infty}\left(z_{\alpha}\left(U_{\alpha}\right), \mathbb{R}\right)$ satisfy

$$
\varphi_{\alpha} \circ f_{\alpha \beta}+\log \left|f_{\alpha \beta}^{\prime}\right|^{2}=\varphi_{\beta} \quad \text { on } \quad z_{\beta}\left(U_{\alpha} \cap U_{\beta}\right) .
$$

According to the uniformization theorem, $X$ has a unique conformal metric of the constant Gaussian curvature -1, called hyperbolic metric. The corresponding functions $\varphi_{\alpha}$ on $z_{\alpha}\left(U_{\alpha}\right)$ satisfy the so-called Liouville equation,

$$
\frac{\partial^{2} \varphi_{\alpha}}{\partial z_{\alpha} \partial \bar{z}_{\alpha}}=\frac{1}{2} e^{\varphi_{\alpha}}
$$

The Lagrangian formulation of the classical Liouville field theory is based on the action functional $S: \mathscr{C} \mathscr{M}(X) \rightarrow \mathbb{R}$, characterized by the property that its unique critical point is the hyperbolic metric on $X$, and the corresponding Euler-Lagrange equation is the Liouville equation. Classical Liouville field theory is conformally invariant. This fundamental property is a manifestation of the fact that the "Liouville field" $e^{\varphi}=\left\{e^{\varphi_{\alpha}}\right\}_{\alpha \in A}$, as it follows from the transformation law (2.1), is a $(1,1)$-tensor on $X$. For the two-dimensional classical field theory conformal invariance implies that the corresponding stress-energy tensor is traceless (see [BPZ84]). In this section 
we recall the definition of the action functional for the Liouville theory, introduce the stress-energy tensor and describe its properties. In Appendix B we show how to derive the stress-energy tensor from the action functional.

2.1. Liouville action functional. It is well-known (see [ZT87c] and the discussion in [TT03a]) that a rigorous definition of the Liouville action functional on a genus $g>1$ Riemann surface is a nontrivial issue. This is due to the fact that the Liouville field $e^{\varphi}$ is a conformal metric on $X$ rather than a function, so that a naive Dirichlet type functional is not well-defined as an integral of a $(1,1)$-form over $X$ when $g>1$. In [ZT87c], this problem was solved by using a global coordinate on $X$ given by the Schottky uniformization. In TT03a, we were able to tackle this problem when a Riemann surface $X$ is equipped with a global coordinate provided by the uniformization of $X$ by a rather general class of Kleinian groups. Here by a global coordinate on a Riemann surface $X$ we understand the complexanalytic covering $J: \Omega \rightarrow X$ of $X$ by a plane domain $\Omega \subset \hat{\mathbb{C}}=\mathbb{C} \cup\{\infty\}$, such that the corresponding group of deck transformations $\Gamma$ is a Kleinian group with the invariant component $\Omega$. For the purposes of this paper, it will be sufficient to consider global coordinates on $X$ given by the Schottky and quasi-Fuchsian uniformizations.

2.1.1. Schottky uniformization. Marked Riemann surface is a compact Riemann surface $X$ of genus $g>1$ equipped with a canonical system of generators $a_{1}, \ldots, a_{g}, b_{1}, \ldots, b_{g}$ of the fundamental group $\pi_{1}\left(X, x_{0}\right)$ (defined up to an inner automorphism). Schottky uniformization of a marked compact Riemann surface $X$ of genus $g$ is a complex-analytic isomorphism $X \simeq \Gamma \backslash \Omega$, where $\Gamma$ is a marked Schottky group - a strictly loxodromic freely generated Kleinian group with a choice of free generators $\gamma_{1}, \ldots, \gamma_{g} \in \operatorname{PSL}(2, \mathbb{C})$ and with the domain of discontinuity $\Omega$. As an abstract group, $\Gamma \simeq \pi_{1}\left(X, x_{0}\right) / \mathcal{N}$, where $\mathcal{N}$ is the smallest normal subgroup in $\pi_{1}\left(X, x_{0}\right)$ containing $a_{1}, \ldots, a_{g}$, and marked generators $\gamma_{1}, \ldots, \gamma_{g}$ correspond to the cosets $b_{1} \mathcal{N}, \ldots, b_{g} \mathcal{N}$. The holomorphic covering map $J_{S}: \Omega \rightarrow X$ provides a marked Riemann surface $X$ with the Schottky global coordinate. It is always assumed that $\Gamma$ is normalized, i.e., the attracting and repelling fixed points of $\gamma_{1}$ are 0 and $\infty$, and the attracting fixed point of $\gamma_{2}$ is 1 . The space $\mathscr{C} \mathscr{M}(X)$ is identified with the affine subspace of $C^{\infty}(\Omega, \mathbb{R})$ consisting of functions $\varphi$ satisfying condition

$$
\varphi \circ \gamma+\log \left|\gamma^{\prime}\right|^{2}=\varphi, \quad \gamma \in \Gamma .
$$

According to ZT87c (see also TT03a for the cohomological interpretation), the Liouville action functional $S: \mathscr{C} \mathscr{M}(X) \rightarrow \mathbb{R}$ is defined by the following formula,

$$
S(\varphi)=\frac{i}{2} \iint_{F} \omega[\varphi]+\frac{i}{2} \sum_{k=2}^{g} \oint_{C_{k}} \theta_{\gamma_{k}^{-1}}[\varphi]+4 \pi \sum_{k=2}^{g} \log \left|c\left(\gamma_{k}\right)\right|^{2} .
$$


Here

$$
\begin{aligned}
\omega[\varphi] & =\left(\left|\varphi_{z}\right|^{2}+e^{\varphi}\right) d z \wedge d \bar{z} \\
\theta_{\gamma^{-1}}[\varphi] & =\left(\varphi-\frac{1}{2} \log \left|\gamma^{\prime}\right|^{2}\right)\left(\frac{\gamma^{\prime \prime}}{\gamma^{\prime}} d z-\frac{\overline{\gamma^{\prime \prime}}}{\overline{\gamma^{\prime}}} d \bar{z}\right),
\end{aligned}
$$

where the subscript $z$ stands for the partial derivative, $c(\gamma)=c$ for $\gamma=\left(\begin{array}{ll}a & b \\ c & d\end{array}\right)$, and $F \subset \Omega$ is a fundamental domain for the marked Schottky group $\Gamma$ - a region bounded by $2 g$ non-intersecting smooth Jordan curves $C_{1}, C_{1}^{\prime}, \ldots, C_{g}, C_{g}^{\prime}$, satisfying $C_{k}^{\prime}=-\gamma_{k}\left(C_{k}\right), k=1, \ldots, g$.

The Liouville action functional satisfies the property

$$
S(\varphi+\chi)-S(\varphi)=\iint_{X}\left(e^{-\varphi}\left|\chi_{z}\right|^{2}+e^{\chi}+K \chi-1\right) e^{\varphi} d^{2} z,
$$

for all $d s^{2}=e^{\varphi}|d z|^{2} \in \mathscr{C} \mathscr{M}(X)$ and $\chi \in C^{\infty}(X, \mathbb{R})$, where $K=-2 e^{-\varphi} \varphi_{z \bar{z}}$ is the Gaussian curvature of the metric $d s^{2}$, and $e^{\varphi} d^{2} z=e^{\varphi} d x \wedge d y, z=x+i y$, is the corresponding area form on $X$ (see [ZT87c and [TT03a, Lemma 2.1]). It follows from (2.5) that $S$ has a unique non-degenerate critical point given by the hyperbolic metric on $X$. We will denote the corresponding solution of the Liouville equation by $\varphi_{c l}$ and, using the physics terminology, will call the corresponding critical value of $S$ the classical action $S_{c l}$. We have for $\chi \in C^{\infty}(X, \mathbb{R})$,

$$
S\left(\varphi_{c l}+\chi\right)-S_{c l}=\iint_{X}\left(e^{-\varphi_{c l}}\left|\chi_{z}\right|^{2}+e^{\chi}-\chi-1\right) e^{\varphi_{c l}} d^{2} z .
$$

The classical action $S_{c l}$ for varying Riemann surfaces defines a function on the Schottky space $\mathfrak{S}_{g}$, and $-S_{c l}$ is a Kähler potential for the Weil-Petersson metric on $\mathfrak{S}_{g}$ [ZT87c].

2.1.2. Fuchsian and quasi-Fuchsian uniformizations. The Fuchsian uniformization of a compact Riemann surface $X$ of genus $g>1$ is a complex-analytic isomorphism $X \simeq \Gamma \backslash \mathbb{U}$, where $\Gamma$ is a torsion-free, strictly hyperbolic Fuchsian group, and $\mathbb{U}$ is the upper half-plane. Equivalently, the Fuchsian uniformization is a holomorphic covering $J_{F}: \mathbb{U} \rightarrow X$, with the group of deck transformations $\Gamma \simeq \pi_{1}\left(X, x_{0}\right)$. It equips the Riemann surface $X$ with the Fuchsian global coordinate, and the space $\mathscr{C} \mathscr{M}(X)$ is identified with the affine subspace of $C^{\infty}(\mathbb{U}, \mathbb{R})$ consisting of functions $\varphi$ satisfying condition (2.3).

The Liouville action functional $S: \mathscr{C} \mathscr{M}(X) \rightarrow \mathbb{R}$ is defined explicitly by the formula similar to (2.4). It is based on the homological algebra machinery associated with the $\Gamma$-action on $\mathbb{U}$, developed in [AT97, and we refer to [TT03a] for the details. As in the Schottky case, the action functional $S$ has a unique non-degenerate critical point given by the hyperbolic metric on $X$, and satisfies property (2.6). It is an easy computation (see [TT03a, Corollary 2.1]) that $S_{c l}=4 \pi(2 g-2)$ - twice the hyperbolic area of $X$. 
To describe the quasi-Fuchsian uniformization of $X$, fix a Riemann surface $Y$ of the same genus as $X$ but with the opposite orientation. According to the Bers' simultaneous uniformization theorem, there exists a quasi-Fuchsian group $\Gamma$ with the domain of discontinuity $\Omega \subset \hat{\mathbb{C}}=\mathbb{C} \cup\{\infty\}$, such that $X \sqcup Y \simeq \Gamma \backslash \Omega$. The group $\Gamma$ is unique up to a conjugation in $\operatorname{PSL}(2, \mathbb{C})$ if $X$ and $Y$ are marked Riemann surfaces, and domain $\Omega$ consists of two disjoint components $\Omega_{1}$ and $\Omega_{2}$, which cover the Riemann surfaces $X$ and $Y$ respectively. The covering $J_{Q F}: \Omega_{1} \rightarrow X$ defines a quasi-Fuchsian global coordinate on $X$ (which depends on $Y$ ).

The definition of the Liouville action functional on the space $\mathscr{C} \mathscr{M}(X \sqcup Y)$ of conformal metrics on $X \sqcup Y$ is a generalization of the Fuchsian case. We refer to [TT03a] for the explicit representation and details. Here we just emphasize that the action functional on $\mathscr{C} \mathscr{M}(X \sqcup Y)$ satisfies property (2.5) and has a unique non-degenerate critical point, given by the hyperbolic metric on $X \sqcup Y$. Moreover, the choice of the hyperbolic metric on $Y$ defines the embedding $\mathscr{C} \mathscr{M}(X) \hookrightarrow \mathscr{C} \mathscr{M}(X \sqcup Y)$, and the restriction of the action functional to $\mathscr{C} \mathscr{M}(X)$ is the Liouiville action functional $S$ for the quasi-Fuchsian global coordinate on $X$, which satisfies property (2.6). Corresponding classical action $S_{c l}$ depends non-trivially on $X$, and for varying $X$ (and fixed $Y$ ) defines a function on the Teichmüller space $\mathfrak{T}_{g}$ of marked compact Riemann surfaces of genus $g>1$. It is proved in TT03a that the function $-S_{c l}$ is a Kähler potential for the Weil-Petersson metric on $\mathfrak{T}_{g}$.

2.2. The stress-energy tensor. The stress-energy tensor is associated with local deformations of classical fields - conformal metrics on $X$, and is defined by corresponding variational derivatives of the action functional (see Appendix B for details). For the classical Liouville theory, its $(2,0)$ and $(0,2)$ components are given by

$$
T(\varphi)=\varphi_{z z}-\frac{1}{2} \varphi_{z}^{2} \quad \text { and } \quad \bar{T}(\varphi)=\overline{T(\varphi)}=\varphi_{\bar{z} \bar{z}}-\frac{1}{2} \varphi_{\bar{z}}^{2}
$$

Here $\varphi$ is a Liouville field - a function $\varphi \in C^{\infty}(\Omega, \mathbb{R})$ satisfying transformation law (2.3). It follows from (2.3) that the function $T(z)=T(\varphi)(z)$ on $\Omega$ satisfies

$$
T \circ \gamma\left(\gamma^{\prime}\right)^{2}=T, \quad \gamma \in \Gamma,
$$

i.e., is a quadratic differential for $\Gamma$. Corresponding classical stress-energy tensor $T_{c l}=T\left(\varphi_{c l}\right)$ satisfies the "conservation law"

$$
\partial_{\bar{z}} T_{c l}=0,
$$

i.e., is a holomorphic quadratic differential for $\Gamma$. This property expresses the fact that the stress-energy tensor for the classical Liouville theory is traceless. The same result holds for Fuchsian and quasi-Fuchsian global coordinates as well and, in particular, for the Fuchsian case $T_{c l}=0$. In this form the stress-energy tensor $T$ for the Liouville theory was introduced by Poincaré Poi98 more than a hundred years ago in his proof of the uniformization theorem for Riemann surfaces which uses the Liouville equation. 
The stress-energy tensor $T$ has the following geometric interpretation. For every $d s^{2}=e^{\varphi}|d z|^{2}=\left\{e^{\varphi_{\alpha}}\left|d z_{\alpha}\right|^{2}\right\}_{\alpha \in A} \in \mathscr{C} \mathscr{M}(X)$ define the following functions on $z_{\alpha}\left(U_{\alpha}\right)$,

$$
T_{\alpha}(\varphi)=\partial_{z_{\alpha}}^{2} \varphi_{\alpha}-\frac{1}{2}\left(\partial_{z_{\alpha}} \varphi_{\alpha}\right)^{2} \quad \text { and } \quad \bar{T}_{\alpha}(\varphi)=\bar{\partial}_{z_{\alpha}}^{2} \varphi_{\alpha}-\frac{1}{2}\left(\bar{\partial}_{z_{\alpha}} \varphi_{\alpha}\right)^{2}
$$

It follows from (2.1) that on every $z_{\beta}\left(U_{\alpha} \cap U_{\beta}\right)$,

$$
T_{\beta}=T_{\alpha} \circ f_{\alpha \beta}\left(f_{\alpha \beta}^{\prime}\right)^{2}+\mathcal{S}\left(f_{\alpha \beta}\right)
$$

where

$$
\mathcal{S}(f)=\frac{f^{\prime \prime \prime}}{f^{\prime}}-\frac{3}{2}\left(\frac{f^{\prime \prime}}{f^{\prime}}\right)^{2}
$$

is the Schwarzian derivative of a holomorphic function $f$. By definition, collection $T(\varphi)=\left\{T_{\alpha}(\varphi)\right\}_{\alpha \in A}$ satisfying (2.8) is a non-holomorphic projective connection on $X$, and it follows from the Liouville equation that $T_{c l}$ is a holomorphic projective connection. Since the hyperbolic metric $e^{\varphi_{c l}}|d z|^{2}$ is a push-forward of the Poincaré metric on $\mathbb{U}$ by the covering map $J_{F}: \mathbb{U} \rightarrow X$, a simple computation gives $T_{c l}=\left\{\mathcal{S}_{z_{\alpha}}\left(J_{F}^{-1}\right)\right\}_{\alpha \in A}$. Using the property $\mathcal{S}(\gamma)=0$ for all $\gamma \in \operatorname{PSL}(2, \mathbb{C})$, and the Caley identity

$$
\mathcal{S}(f \circ g)=\mathcal{S}(f) \circ g\left(g^{\prime}\right)^{2}+\mathcal{S}(g),
$$

it is easy to verify directly that $\mathcal{S}_{z_{\alpha}}\left(J_{F}^{-1}\right)$ are well-defined functions on $z_{\alpha}\left(U_{\alpha}\right)$, which satisfy (2.8). Slightly abusing notations, we will write $T_{c l}=$ $\mathcal{S}\left(J_{F}^{-1}\right)$.

Let $z_{K}$ be a global coordinate on $X$ given by the covering $J_{K}: \Omega_{K} \rightarrow X$, and let $e^{\varphi_{K}}\left|d z_{K}\right|^{2}=J_{K}^{*}\left(d s^{2}\right)$ be the pull-back of $d s^{2}=e^{\varphi}|d z|^{2} \in \mathscr{C} \mathscr{M}(X)$ by $J_{K}$. From (2.7) we obtain

$$
T(\varphi)=T\left(\varphi_{K}\right) \circ J_{K}^{-1}\left(J_{K}^{-1}\right)_{z}^{2}+\mathcal{S}\left(J_{K}^{-1}\right),
$$

where $z$ is a local coordinate on $X$. Thus the push-forward to $X$ of the quadratic differential $T\left(\varphi_{K}\right)$ on $\Omega_{K}$ is not a quadratic differential on $X$, but a projective connection. The stress-energy tensor also behaves like a projective connection under changes of global coordinates. Namely, consider the following commutative diagram

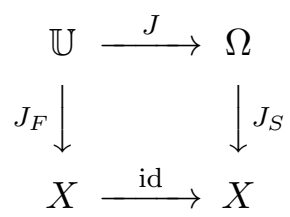

where $J=J_{S}^{-1} \circ J_{F}$ describes the relation between Fuchsian and Schottky global coordinates. Denoting by $e^{\varphi_{F}}\left|d z_{F}\right|^{2}$ and $e^{\varphi_{S}}\left|d z_{S}\right|^{2}$ the pull-backs of $d s^{2}=e^{\varphi}|d z|^{2} \in \mathscr{C} \mathscr{M}(X)$ by the mappings $J_{F}$ and $J_{S}$ respectively, we obtain

$$
T\left(\varphi_{S}\right)=T\left(\varphi_{F}\right) \circ J^{-1}\left(J_{z}^{-1}\right)^{2}+\mathcal{S}\left(J^{-1}\right) .
$$


In particular, $T_{c l}=\mathcal{S}\left(J^{-1}\right)$. The same formula (2.10) holds if we replace $J_{S}$ by a covering $J_{K}: \Omega_{K} \rightarrow X$ associated with any global coordinate $z_{K}$ on $X$, e.g., by $J_{K}=J_{Q F}$.

For every $\chi \in C^{\infty}(X)$ set $\chi_{\alpha}=\chi \circ z_{\alpha}^{-1}$ and let $q_{\alpha}=\left(\partial_{z_{\alpha}} \chi_{\alpha}\right)^{2} \in$ $C^{\infty}\left(z_{\alpha}\left(U_{\alpha}\right)\right)$. On every $z_{\beta}\left(U_{\alpha} \cap U_{\beta}\right)$ these functions satisfy

$$
q_{\beta}=q_{\alpha} \circ f_{\alpha \beta}\left(f_{\alpha \beta}^{\prime}\right)^{2},
$$

so that the collection $q=\left\{q_{\alpha}\right\}_{\alpha \in A}$ is a quadratic differential on $X$. If $z$ is a local coordinate on $X$, then $q=\chi_{z}^{2}$. Now let $\mathcal{D}_{\alpha}$ be the following second order differential operator acting on functions on $z_{\alpha}\left(U_{\alpha}\right)$,

$$
\mathcal{D}_{\alpha}=e^{\varphi_{c l}} \circ \partial_{z_{\alpha}} \circ e^{-\varphi_{c l}} \circ \partial_{z_{\alpha}}=\partial_{z_{\alpha}}^{2}-\left(\partial_{z_{\alpha}} \varphi_{c l}\right) \partial_{z_{\alpha}} .
$$

It follows from (2.1) that for every $\chi \in C^{\infty}(X)$ the collection $\mathcal{D} \chi=\left\{\mathcal{D}_{\alpha} \chi_{\alpha}\right\}_{\alpha \in A}$ is also a quadratic differentia ${ }^{3}$ on $X$. If $z$ is a local coordinate on $X$, then

$$
\mathcal{D}_{z} \chi=e^{\varphi_{c l}}\left(e^{-\varphi_{c l}} \chi_{z}\right)_{z}=\chi_{z z}-\left(\partial_{z} \varphi_{c l}\right) \chi_{z} .
$$

For every $e^{\varphi}|d z|^{2} \in \mathscr{C} \mathscr{M}(X)$ setting $\varphi=\varphi_{c l}+\chi$, where $\chi \in C^{\infty}(X, \mathbb{R})$, we get

$$
T(\varphi)=T_{c l}+\mathcal{D}_{z} \chi-\frac{1}{2} \chi_{z}^{2}
$$

Quadratic differentials $\mathcal{D}_{z} \chi-\frac{1}{2} \chi_{z}^{2}$ for $\chi \in C^{\infty}(X, \mathbb{R})$ describe "fluctuations" around the classical stress-energy tensor $T_{c l}$.

\section{Quantum LiOUVILle TheORY}

Here we formulate quantum Liouville theory using the Feynman functional integral formalism. The space $\mathscr{C} \mathscr{M}(X)$ of conformal metrics on $X$ is an infinite-dimensional Fréchet manifold with a natural Riemannian metric defined by

$$
\|\delta \varphi\|^{2}=\iint_{X}|\delta \varphi|^{2} e^{\varphi} d^{2} z, \quad \delta \varphi \in T_{\varphi} \mathscr{C} \mathscr{M}(X) \simeq C^{\infty}(X, \mathbb{R}) .
$$

Assuming that the metric $\|\cdot\|^{2}$ gives rise to the "volume element" $\mathscr{D} \varphi$ and choosing a global coordinate on $X$ (Schottky, or quasi-Fuchsian), we define the partition function $\langle X\rangle$ — "expectation value" of the Riemann surface $X$, by the following functional integral

$$
\langle X\rangle=\int_{\mathscr{C} \mathscr{M}(X)} e^{-\frac{1}{2 \pi \hbar} S(\varphi)} \mathscr{D} \varphi .
$$

Here the dimensionless parameter $\hbar>0$ plays the role of the Planck constant. For varying $X$ the partition function $\langle X\rangle$ gives rise to a real-valued function on the corresponding deformation space, Schottky space $\mathfrak{S}_{g}$, or Teichmüller space $\mathfrak{T}_{g}$, defined in Section 4.1. The correlation functions of

\footnotetext{
${ }^{3}$ This is true for every $\varphi$ satisfying (2.1).
} 
multi-local fields $\mathcal{O}$ - functionals on $\mathscr{C} \mathscr{M}(X)$ which depend on the values of $\varphi$ at finitely many points on $X$, are defined by

$$
\langle\mathcal{O} X\rangle=\int_{\mathscr{C} \mathscr{M}(X)} \mathcal{O}(\varphi) e^{-\frac{1}{2 \pi \hbar} S(\varphi)} \mathscr{D} \varphi
$$

For $\mathcal{O}=\prod_{i=1}^{k} T(\varphi)\left(z_{i}\right) \prod_{j=1}^{l} \bar{T}(\varphi)\left(\bar{w}_{j}\right)$, where $z$ is a global coordinate on $X$, we get multi-point correlation functions with insertions of the stress-energy tensor. Correlation functions $\left\langle\prod_{i=1}^{k} T(\varphi)\left(z_{i}\right) \prod_{j=1}^{l} \bar{T}(\varphi)\left(\bar{w}_{j}\right) X\right\rangle$ are tensors of type $(2,0)$ in $z_{1}, \ldots, z_{k}$, and tensors of type $(0,2)$ in $w_{1}, \ldots, w_{l}$, and are symmetric with respect to these two groups of variables.

Here we do not attempt to give a rigorous mathematical definition of functional integrals (3.1) and (3.2) $)^{4}$. Instead, we define (3.1) and (3.2) perturbatively using background field formalism - the expansion around the critical point of the action, i.e., around the classical solution $\varphi_{c l}$. The result is a formal power series in $\hbar$ with coefficients given by the Feynman rules. The combinatorics of the perturbative expansion in QFT is well-established (see, e.g., Ram90, Wit99] and [Kaz99] for mathematically oriented exposition). Here we describe the formal power series in $\hbar$ for partition and correlation functions, and give a rigorous regularization scheme for the coefficients of these series.

3.1. Feynman rules for the partition function. Let $\Delta_{0}$ be the Laplace operator of the hyperbolic metric acting on functions on $X$,

$$
\Delta_{0}=-e^{-\varphi_{c l}} \frac{\partial^{2}}{\partial z_{\alpha} \partial \bar{z}_{\alpha}} \quad \text { on } \quad z_{\alpha}\left(U_{\alpha}\right)
$$

The Laplacian $\Delta_{0}$ on a compact Riemann surface $X$ is a positive, elliptic operator. Let

$$
G=\frac{1}{2}\left(\Delta_{0}+\frac{1}{2}\right)^{-1}
$$

be one-half of the resolvent of $\Delta_{0}$ at the point $-\frac{1}{2}$. It is well-known that $G$ is an integral operator with a point-wise positive kernel $G(P, Q)$, which is a smooth function on $X \times X$, except for the diagonal $P=Q$, where it has a logarithmic singularity. The function $G(P, Q)$ is called the propagator of the quantum Liouville theory. We describe its properties in Section 5.

\footnotetext{
${ }^{4}$ This would require rigorous definition of the probability measure on the space of distributions on $\mathscr{C} \mathscr{M}(X)$, similar to what has been done in constructive quantum field theory in two dimensions [Sim74, GJ87.
} 
The perturbative expansion of Feynman integrals is based on Wick's theorem - the following formula for Gaussian integrals,

$$
\begin{gathered}
\int_{C^{\infty}(X, \mathbb{R})} \chi\left(P_{1}\right) \ldots \chi\left(P_{n}\right) e^{-\frac{1}{2} \iint_{X} \chi\left(\Delta_{0}+\frac{1}{2}\right) \chi e^{\varphi} c l d^{2} z} \mathscr{D} \chi \\
= \begin{cases}0, & \text { if } n \text { is odd, } \\
\frac{D}{\left(\frac{n}{2}\right) !} \sum_{\sigma \in S_{n}} G\left(P_{\sigma(1)}, P_{\sigma(2)}\right) \ldots G\left(P_{\sigma(n-1)}, P_{\sigma(n)}\right), & \text { if } n \text { is even. }\end{cases}
\end{gathered}
$$

Here $P_{1}, \ldots, P_{n}$ are distinct points on $X, D^{-2}=\operatorname{det}\left(\Delta_{0}+\frac{1}{2}\right)$ is the zetafunction regularized determinant of $\Delta_{0}+\frac{1}{2}$, and $S_{n}$ is the permutation group on $n$ elements. The integration measure $\mathscr{D} \chi$ is defined as the volume form of the Riemannian metric

$$
\|\chi\|^{2}=\iint_{X}|\chi|^{2} e^{\varphi_{c l}} d^{2} z
$$

on $C^{\infty}(X, \mathbb{R})$. Effectively, formula (3.3) is a definition of the Gaussian measure $\mathscr{D} \chi$ (see, e.g., GJ87]).

To use (3.3), we expand $\varphi$ around the critical point $\varphi_{c l}$,

$$
\varphi=\varphi_{c l}+\sqrt{\pi \hbar} \chi
$$

where $\chi \in C^{\infty}(X, \mathbb{R})$. By (2.6) we have

$$
S\left(\varphi_{c l}+\chi\right)=S_{c l}+\pi \hbar \iint_{X} \chi\left(\Delta_{0}+\frac{1}{2}\right) \chi e^{\varphi_{c l}} d^{2} z+\sum_{n=3}^{\infty} \frac{(\sqrt{\pi \hbar})^{n}}{n !} \iint_{X} \chi^{n} e^{\varphi_{c l}} d^{2} z .
$$

Substituting this expansion into (3.1) and using $\mathscr{D} \varphi=\mathscr{D} \chi$ (which may be considered as a perturbative definition of $\mathscr{D} \varphi$ ), we obtain

$$
\langle X\rangle=e^{-\frac{1}{2 \pi \hbar} S_{c l}} \sum_{\mathbf{m}} c_{\mathbf{m}} \int_{C^{\infty}(X, \mathbb{R})} \prod_{n=1}^{\infty}\left(\iint_{X} \chi^{n} e^{\varphi_{c l}} d^{2} z\right)^{m_{n}} e^{-\|\chi\|_{2}^{2}} \mathscr{D} \chi,
$$

where

$$
\|\chi\|_{2}^{2}=\frac{1}{2} \iint_{X} \chi\left(\Delta_{0}+\frac{1}{2}\right) \chi e^{\varphi_{c l}} d^{2} z
$$

is essentially a Sobolev norm square of a function $\chi$. The summation in (3.4) goes over all multi-indices $\mathbf{m}=\left(m_{1}, m_{2}, \ldots\right)$, such that $m_{n} \geq 0, m_{1}=$ $m_{2}=0, m_{n}=0$ for $n>N$ for some $N>0$, and

$$
c_{\mathbf{m}}=\frac{(\pi \hbar)^{\frac{|\tilde{\mathbf{m}}|}{2}}}{(-2)^{|\mathbf{m}|} \mathbf{m} ! \mathbf{n}^{\mathbf{m} !}},
$$


where

$$
\begin{aligned}
|\mathbf{m}| & =\sum_{n=1}^{\infty} m_{n}, & |\tilde{\mathbf{m}}| & =\sum_{n=1}^{\infty}(n-2) m_{n} \\
\mathbf{m} ! & =\prod_{n=1}^{\infty} m_{n} !, & \mathbf{n}^{\mathbf{m}} ! & =\prod_{n=1}^{\infty}(n !)^{m_{n}}
\end{aligned}
$$

Using (3.3) and standard combinatorics of functional integration (see, e.g, [Kaz99, Wit99]), it is easy to convert (3.4) into the following sum over Feynman diagrams,

$$
\langle X\rangle=D e^{-\frac{1}{2 \pi \hbar} S_{c l}}\left(1+\sum_{\Upsilon \in \mathcal{G} \geq 3}(-1)^{|V(\Upsilon)|}(2 \pi \hbar)^{-\chi(\Upsilon)} \frac{W_{\Upsilon}(X)}{\mid \text { Aut } \Upsilon \mid}\right) .
$$

Here $\mathcal{G}_{\geq 3}$ is a set of graphs $\Upsilon$ with all vertices of valency $\geq 3$. For $\Upsilon \in \mathcal{G}_{\geq 3}$ $V(\Upsilon)$ and $E(\Upsilon)$ are, respectively, the set of vertices 5 and the set of edges of a graph $\Upsilon$, and $|V(\Upsilon)|=\# V(\Upsilon),|E(\Upsilon)|=\# E(\Upsilon)$. Also, $\mid$ Aut $\Upsilon \mid$ is the order of the group of automorphisms of $\Upsilon$, and

$$
\chi(\Upsilon)=|V(\Upsilon)|-|E(\Upsilon)|=m-|L(\Upsilon)|
$$

is the Euler characteristic of $\Upsilon$, where $|L(\Upsilon)|$ is the number of loops of $\Upsilon$, and $m$ is the number of connected components of $\Upsilon$. The weights $W_{\Upsilon}(X)$ are given by the following formula,

$$
W_{\Upsilon}(X)=\int \ldots \int H\left(P_{1}, \ldots, P_{V}\right) \prod_{k=1}^{V} d P_{k} .
$$

Here $V=|V(\Upsilon)|, d P_{k}=e^{\varphi_{c l}\left(z_{k}\right)} d^{2} z_{k}$ is the area form of the hyperbolic metric on the $k$-th factor in $X^{V}=\underbrace{X \times \cdots \times X}_{V \text { times }}$, and

$$
H\left(P_{1}, \ldots, P_{V}\right)=\prod_{e \in E(\Upsilon)} G\left(P_{v_{0}(e)}, P_{v_{1}(e)}\right),
$$

where $\partial e=\left\{v_{0}(e), v_{1}(e)\right\} \subset V(\Upsilon)$ are the endpoints of the edge $e \in E(\Upsilon)$, and $G(P, Q)$ is the propagator.

Formulas (3.5)-(3.7) give a formal definition of the partition function $\langle X\rangle$. However, for graphs with self-loops, i.e., graphs having edges that start and end at the same vertex, corresponding weights are infinite, since the propagator $G(P, Q)$ diverges at $Q=P$. To make sense of the formal power series expansion (3.5), one needs to redefine the propagator at coincident points. It follows from the short-distance behavior of the resolvent kernel in Section [5.1, that the following expression

$$
G(P, P):=\lim _{Q \rightarrow P}\left(G(P, Q)+\frac{1}{2 \pi}\left(\log |z(P)-z(Q)|^{2}+\varphi_{c l}(z(P))\right)\right)
$$

\footnotetext{
${ }^{5}$ By definition, the set $V(\Upsilon)$ is not empty.
} 
where $z$ is a local coordinate in the neighborhood $U \subset X$ containing $P$ and $Q$, defines a smooth real-valued function on $X$. It is this "regularization at the coincident points" (see Tak93, Tak96a]) that we use in (3.7).

As it is customary in quantum field theory, we introduce the free energy

$$
\mathcal{F}_{X}=-\log \langle X\rangle \text {. }
$$

It is well-known (see, e.g., Ram90, Kaz99, Wit99]) that passing from the partition function to free energy results in replacing the sum over all graphs in the expansion (3.5) by the sum over connected graphs only.

Definition 3.1. The free energy $\mathcal{F}_{X}=-\log \langle X\rangle$ of the quantum Liouville theory on a compact Riemann surface $X$ in the background field formalism is given by the following formal power series in $\hbar$,

$$
\mathcal{F}_{X}=\frac{1}{2 \pi \hbar} S_{c l}+\frac{1}{2} \log \operatorname{det}\left(\Delta_{0}+\frac{1}{2}\right)-\sum_{\Upsilon \in \mathcal{G}_{\geq 3}^{(c)}}(-1)^{|V(\Upsilon)|}(2 \pi \hbar)^{-\chi(\Upsilon)} \frac{W_{\Upsilon}(X)}{\mid \text { Aut } \Upsilon \mid}
$$

where $\mathcal{G}_{\geq 3}^{(c)}$ is a subset of all connected graphs $\Upsilon \in \mathcal{G}_{\geq 3}$.

Remark 3.2. The term of order $\hbar^{-1}$ in $\mathcal{F}_{X}$ represents classical contribution to the free energy. The constant in $\hbar$ term is a 1-loop contribution associated with the circle diagram. By definition, it is equal to one-half of the logarithm of the regularized determinant of the elliptic operator $\Delta_{0}+\frac{1}{2}$. The higher order terms correspond to graphs with loops: the $n$-loop term - the coefficient in front of $\hbar^{n-1}$ - is the contribution of all connected graphs with $n$ loops in $\mathcal{G}_{\geq 3}$.

Remark 3.3. It follows from Definition 3.1 that different choices of global coordinates on $X$ affect only classical contribution to the free energy. All other terms in the perturbative expansion of $\mathcal{F}_{X}$ are canonical in the sense that they only depend on the hyperbolic metric on $X$ through the resolvent kernel $G(P, Q)$. In what follows it will be convenient, though not really necessary, to consider Schottky, quasi-Fuchsian and Fuchsian global coordinates on $X$. In Section 9 we will interpret the free energy in terms of complex geometry of the moduli space $\mathfrak{M}_{g}$.

Remark 3.4. According to DP86] and Sar87, the 1-loop contribution, up to an additive constant $c_{g}$ depending only on the genus $g$, can be expressed solely in terms of the hyperbolic geometry of $X$ as follows,

$$
\log \operatorname{det}\left(\Delta_{0}+\frac{1}{2}\right)=\log Z_{X}(2)+c_{g} .
$$

Here $Z_{X}(s)$ is the Selberg zeta function of a Riemann surface $X$, defined for $\operatorname{Re} s>1$ by the following absolutely convergent product:

$$
Z_{X}(s)=\prod_{\{\ell\}} \prod_{n=0}^{\infty}\left(1-e^{-(s+n)|\ell|}\right)
$$

where $\ell$ runs through the set of all simple closed oriented geodesics on $X$ with respect to the hyperbolic metric, and $|\ell|$ is the length of $\ell$. 
3.2. Feynman rules for correlation functions. Let $z$ be a global coordinate on $X$. Here we define the multi-point correlation functions

$$
\left\langle\prod_{i=1}^{k} T\left(z_{i}\right) \prod_{j=1}^{l} \bar{T}\left(\bar{w}_{j}\right) X\right\rangle=\int_{\mathscr{C} \mathscr{\mathscr { M }}(X)} \prod_{i=1}^{k} T(\varphi)\left(z_{i}\right) \prod_{j=1}^{l} \bar{T}(\varphi)\left(\bar{w}_{j}\right) e^{-\frac{1}{2 \pi \hbar} S(\varphi)} \mathscr{D} \varphi
$$

as formal power series in $\hbar$. It will be convenient to replace $T(\varphi)$ and $\bar{T}(\varphi)$ by $\frac{1}{\hbar} T(\varphi)$ and $\frac{1}{\hbar} \bar{T}(\varphi)$ respectively, and in what follows we will always use this normalization. As in the definition of the partition function $\langle X\rangle$, we use the substitution $\varphi=\varphi_{c l}+\sqrt{\pi \hbar} \chi$. It follows from (2.11) that

$$
T(\varphi)=T_{c l}+\sqrt{\frac{\pi}{\hbar}} \mathcal{D}_{z} \chi-\frac{\pi}{2} \chi_{z}^{2}
$$

Using (3.3) we get that Feynman diagrams for $\left\langle\prod_{i=1}^{k} T\left(z_{i}\right) \prod_{j=1}^{l} \bar{T}\left(\bar{w}_{j}\right) X\right\rangle$ are labeled graphs with $k+l$ vertices with valencies 1 and 2 carrying the labels $z_{1}, \ldots, z_{k}, \bar{w}_{1}, \ldots, \bar{w}_{l}$, and with all other vertices of valency $\geq 3$. In order to sum only over connected graphs, we introduce irreducible correlation functions,

$$
\begin{aligned}
\langle\langle T(z) X\rangle\rangle & =\frac{\langle T(z) X\rangle}{\langle X\rangle}, \quad\langle\langle\bar{T}(\bar{z}) X\rangle\rangle=\frac{\langle\bar{T}(\bar{z}) X\rangle}{\langle X\rangle}, \\
\langle\langle T(z) T(w) X\rangle\rangle & =\frac{\langle T(z) T(w) X\rangle}{\langle X\rangle}-\langle\langle T(z) X\rangle\rangle\langle\langle T(w) X\rangle\rangle, \\
\langle\langle T(z) \bar{T}(\bar{w}) X\rangle\rangle & =\frac{\langle T(z) \bar{T}(\bar{w}) X\rangle}{\langle X\rangle}-\langle\langle T(z) X\rangle\rangle\langle\langle\bar{T}(\bar{w}) X\rangle\rangle .
\end{aligned}
$$

In general, denoting $I=\prod_{i=1}^{k} T\left(z_{i}\right) \prod_{j=1}^{l} \bar{T}\left(\bar{w}_{j}\right)$, we have inductively (see, e.g., Tak96a]

$$
\langle\langle I X\rangle\rangle=\frac{\langle I X\rangle}{\langle X\rangle}-\sum_{r} \sum_{I=I_{1} \ldots I_{r}}\left\langle\left\langle I_{1} X\right\rangle\right\rangle \ldots\left\langle\left\langle I_{r} X\right\rangle\right\rangle,
$$

where the sum goes over all representations of $I$ as a product of $I_{1}, \ldots, I_{r}$ corresponding to the partition of the set $\left\{z_{1}, \ldots, z_{k}, \bar{w}_{1}, \ldots, \bar{w}_{l}\right\}$ into $r$ nonempty subsets.

The perturbative expansion for the one-point irreducible correlation function with the $(2,0)$ component of the stress-energy tensor is given by

$$
\langle\langle T(z) X\rangle\rangle=T_{c l}(z)+2 \pi \sum_{\Upsilon \in \mathcal{G}_{\{z\}}^{(c)}}(-1)^{|V(\Upsilon)|+\varepsilon_{1}(\Upsilon)}(2 \pi \hbar)^{-\chi(\Upsilon)} \frac{W_{\Upsilon}(X ; z)}{\mid \text { Aut } \Upsilon \mid} .
$$

Here $\mathcal{G}_{\{z\}}^{(c)}$ is the set of all connected graphs $\Upsilon$ with a single vertex of valency 1 or 2 with the label $z$ and all other vertices of valency $\geq 3$, and $\varepsilon_{1}(\Upsilon)=1$ or 0 depending on whether the labeled vertex has valency 1 or 2 . Also Aut $\Upsilon$ is the group of automorphisms of $\Upsilon$ which preserves the labeling, and $\chi(\Upsilon)=|V(\Upsilon)|-|E(\Upsilon)|=1-|L(\Upsilon)|$ is the Euler characteristic of $\Upsilon$, where 
$|L(\Upsilon)|$ is the number of loops of $\Upsilon$. The weights $W_{\Upsilon}(X ; z)$ are given by the following formula,

$$
W_{\Upsilon}(X ; z)=\int \ldots \int_{X^{\tilde{V}}} \ldots H\left(P_{1}, \ldots, P_{V}\right) \prod_{k=1}^{\tilde{V}} d P_{k},
$$

where $\tilde{V}=|V(\Upsilon)|-1, P_{V}=z$ corresponds to vertex $v$ labeled by $z$, and

$$
H\left(P_{1}, \ldots, P_{V}\right)=\prod_{e \in E(\Upsilon)} \mathcal{D}_{v_{0}(e)} \mathcal{D}_{v_{1}(e)} G\left(P_{v_{0}(e)}, P_{v_{1}(e)}\right)
$$

Here

$$
\begin{cases}\mathcal{D}_{v}=\mathrm{id} & \text { if vertex } v \text { has valency } \geq 3 \\ \mathcal{D}_{v}=\mathcal{D}_{z} & \text { if vertex } v \text { has valency } 1 \\ \mathcal{D}_{v}=\partial_{z} & \text { if vertex } v \text { has valency } 2\end{cases}
$$

For self-loops we use the same regularization (3.8) at coincident points, except for the case when vertex $v$ has $n$ self-loops and is connected by an edge to a labeled vertex of valency 1 . In this case we replace one of the factors in $G\left(P_{v}, P_{v}\right)^{n}$ by $G\left(P_{v}, P_{v}\right)+\frac{n}{2 \pi}$. The contribution of the vertex of valency 1 with label $z$ to (3.11) is the factor $\mathcal{D}_{z} G(z, w)$, which has a singularity of the form $1 /(z-w)^{2}$ as $w \rightarrow z$. The same singularity arises when the labeled vertex of valency 2 is attached to a self-loop. The corresponding integrals in (3.10) are understood in the principal value sense.

To complete this definition, we need to assign the weight to a tadpole graph (see Fig. 1)

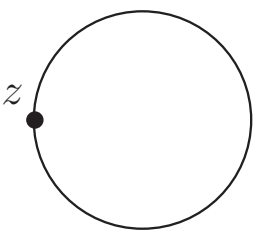

Figure 1

According to [Tak96a], we define

$$
\begin{aligned}
H(z) & =\partial_{z} \partial_{z} G(z, z) \\
& :=\lim _{w \rightarrow z}\left(\partial_{z} \partial_{w} G(z, w)+\frac{1}{2 \pi}\left(\frac{1}{(z-w)^{2}}-\frac{1}{2} e^{\varphi_{c l}(z)} \frac{\bar{z}-\bar{w}}{z-w}\right)\right) .
\end{aligned}
$$

We will show in Section 5.1 that $H(z)$ is a quadratic differential for a group $\Gamma$ corresponding to a global coordinate $z$, which behaves like a projective connection under changes of global coordinates. Analyzing formula (3.9) it is easy to see that $\langle\langle T(z) X\rangle\rangle$ is a formal power series in $\hbar$ whose coefficients are quadratic differentials for $\Gamma$. Except the classical and 1-loop terms, their push-forwards to $X$ are quadratic differentials. In other words, higher loop terms in $\langle\langle T(z) X\rangle\rangle$ do not depend on the choice of global coordinate on $X$. 
The correlation function $\langle\langle\bar{T}(\bar{z}) X\rangle\rangle$ is defined similarly, with $z$ replaced by $\bar{z}$. The perturbative definition of multi-point correlation functions is the following.

Definition 3.5. Irreducible multi-point correlation functions with insertions of the stress-energy tensor are given by the following formal power series in $\hbar$,

$\left\langle\left\langle\prod_{i=1}^{k} T\left(z_{i}\right) \prod_{j=1}^{l} \bar{T}\left(\bar{w}_{j}\right) X\right\rangle\right\rangle=(2 \pi)^{k+l} \sum_{\Upsilon \in \mathcal{G}_{\mathcal{I}}^{(c)}}(-1)^{|V(\Upsilon)|+\varepsilon_{1}(\Upsilon)}(2 \pi \hbar)^{-\chi(\Upsilon)} \frac{W_{\Upsilon}(X ; \mathcal{I})}{\mid \text { Aut } \Upsilon}$.

Here $\mathcal{G}_{\mathcal{I}}^{(c)}$ is the set of all connected graphs $\Upsilon$ with $k+l$ vertices of valencies 1 or 2 labeled by the set $\mathcal{I}=\left\{z_{1}, \ldots, z_{k}, \bar{w}_{1}, \ldots, \bar{w}_{l}\right\}$ and with all other vertices of valency $\geq 3, \varepsilon_{1}(\Upsilon)$ is the number of vertices of valency 1 , and Aut $\Upsilon$ is the group of automorphisms of $\Upsilon$ which preserve the labeling. The weights $W_{\Upsilon}(X ; \mathcal{I})$ are given by

$$
W_{\Upsilon}(X ; \mathcal{I})=\int \ldots \int H\left(P_{1}, \ldots, P_{V}\right) \prod_{k=1}^{\tilde{V}} d P_{k},
$$

where $\tilde{V}=|V(\Upsilon)|-k-l$. Here for a labeled vertex $v$ the point $P_{v}$ is from the labels set $\mathcal{I}$, and

$$
H\left(P_{1}, \ldots, P_{\tilde{V}} ; \mathcal{I}\right)=\prod_{e \in E(\Upsilon)} \mathcal{D}_{v_{0}(e)} \mathcal{D}_{v_{1}(e)} G\left(P_{v_{0}(e)}, P_{v_{1}(e)}\right)
$$

where

$$
\begin{cases}\mathcal{D}_{v}=\text { id } & \text { if } v \text { has valency } \geq 3 \\ \mathcal{D}_{v}=\mathcal{D}_{z_{i}} & \text { if } v \text { has valency } 1 \text { and label } z_{i} \\ \mathcal{D}_{v}=\partial_{z_{i}} & \text { if } v \text { has valency } 2 \text { and label } z_{i} \\ \mathcal{D}_{v}=\mathcal{D}_{\bar{w}_{j}} & \text { if } v \text { has valency } 1 \text { and label } \bar{w}_{j} \\ \mathcal{D}_{v}=\partial_{\bar{w}_{j}} & \text { if } v \text { has valency } 2 \text { and label } \bar{w}_{j}\end{cases}
$$

For graphs with self-loops the weights are regularized by (3.8), except for the case when a vertex $v$ with $n$ self-loops is connected by an edge to a vertex of valency 1 , in which case one of the factors in $G\left(P_{v}, P_{v}\right)^{n}$ is replaced by $G\left(P_{v}, P_{v}\right)+\frac{n}{2 \pi}$. For the cases $k=1, l=0$ and $k=0, l=1$ one should add, correspondingly, the tree-level terms

$$
T_{c l}(z)=\frac{1}{\hbar}\left(\partial_{z}^{2} \varphi_{c l}-\frac{1}{2}\left(\partial_{z} \varphi_{c l}\right)^{2}\right) \quad \text { and } \quad T_{c l}(\bar{w})=\frac{1}{\hbar}\left(\partial_{\bar{w}}^{2} \varphi_{c l}-\frac{1}{2}\left(\partial_{\bar{w}} \varphi_{c l}\right)^{2}\right) .
$$

The weights for the tadpole graphs - graphs with a single vertex of valency 2 labeled by $z$ or $\bar{w}$, are given, correspondingly, by $H(z)$ and $\bar{H}(\bar{w})=\overline{H(w)}$, where $H(z)$ is defined in (3.12).

It follows from Definition 3.5 that $\left\langle\left\langle\prod_{i=1}^{k} T\left(z_{i}\right) \prod_{j=1}^{l} \bar{T}\left(\bar{w}_{j}\right) X\right\rangle\right\rangle$ are symmetric with respect to the variables $z_{1}, \ldots, z_{k}$ and $\bar{w}_{1}, \ldots, \bar{w}_{l}$ respectively. When $k+l \geq 2$, all coefficients in these formal power series are quadratic 
differentials for $\Gamma$ in variables $z_{i}$ and $\bar{w}_{j}$, whose push-forward to $X$ are quadratic differentials 6 . In other words, for $k+l \geq 2$ correlation functions $\left\langle\left\langle\prod_{i=1}^{k} T\left(z_{i}\right) \prod_{j=1}^{l} \bar{T}\left(\bar{w}_{j}\right) X\right\rangle\right\rangle$ do not depend on the choice of a global coordinate on $X$. We will prove in Sections 6 8 that these correlation functions are quadratic differentials in $z_{i}$ and $\bar{w}_{j}$, which are meromorphic in $z_{i}$ and anti-meromorphic in $w_{j}$, and with only poles at coincident points.

\section{Deformation theory}

For the convenience of the reader, here we present necessary basic facts from deformation theory (see e.g., Ahl87, Ber70, Ahl61 or our discussion in [TT03a] $)$.

4.1. Schottky and Teichmüller spaces. Let $\Gamma$ be either a Schottky group, or a quasi-Fuchsian group 7 , with the domain of discontinuity $\Omega$. Let $\mathcal{A}^{-1,1}(\Gamma)$ be a space of bounded Beltrami differentials for $\Gamma$ - the Banach space of $\mu \in L^{\infty}(\mathbb{C})$ satisfying

$$
\mu(\gamma z) \frac{\overline{\gamma^{\prime}(z)}}{\gamma^{\prime}(z)}=\mu(z) \quad \text { for } z \in \Omega, \gamma \in \Gamma
$$

and let $\mathcal{B}^{-1,1}(\Gamma)$ be the open unit ball in $\mathcal{A}^{-1,1}(\Gamma)$ with respect to $\|\cdot\|_{\infty}$ norm,

$$
\|\mu\|_{\infty}=\sup _{z \in \mathbb{C}}|\mu(z)|<1 .
$$

For every $\mu \in \mathcal{B}^{-1,1}(\Gamma)$ there exists a unique quasiconformal homeomorphism $f^{\mu}: \hat{\mathbb{C}} \rightarrow \hat{\mathbb{C}}$ satisfying the Beltrami equation

$$
f_{\bar{z}}^{\mu}=\mu f_{z}^{\mu}
$$

and fixing the points 0,1 and $\infty$. Set $\Gamma^{\mu}=f^{\mu} \circ \Gamma \circ\left(f^{\mu}\right)^{-1}$ and define the deformation space of $\Gamma$ to be

$$
\mathfrak{D}(\Gamma)=\mathcal{B}^{-1,1}(\Gamma) / \sim,
$$

where $\mu \sim \nu$ if and only if $f^{\mu}=f^{\nu}$ on $\hat{\mathbb{C}} \backslash \Omega$, which is equivalent to the condition $f^{\mu} \circ \gamma \circ\left(f^{\mu}\right)^{-1}=f^{\nu} \circ \gamma \circ\left(f^{\nu}\right)^{-1}$ for all $\gamma \in \Gamma$.

The deformation space $\mathfrak{D}(\Gamma)$ has a natural structure of a complex manifold, explicitly described as follows (see, e.g., Ahl87]). Let $\mathcal{H}^{-1,1}(\Gamma)$ be the Hilbert space of Beltrami differentials for $\Gamma$ with the inner product

$$
\left(\mu_{1}, \mu_{2}\right)=\iint_{F} \mu_{1}(z) \overline{\mu_{2}(z)} \rho(z) d^{2} z,
$$

\footnotetext{
${ }^{6}$ Here by a quadratic differential in $\bar{w}$ we understand a complex-conjugate of a quadratic differential in $w$.

${ }^{7}$ In fact, $\Gamma$ could be any non-elementary, finitely generated Kleinian group.
} 
where $\mu_{1}, \mu_{2} \in \mathcal{H}^{-1,1}(\Gamma), F$ is a fundamental domain for $\Gamma$ in $\Omega$, and $\rho=e^{\varphi_{c l}}$ is density of the hyperbolic metric on $\Omega$. Denote by $\Omega^{-1,1}(\Gamma)$ the finitedimensional subspace of harmonic Beltrami differentials with respect to the hyperbolic metric. It consists of $\mu \in \mathcal{H}^{-1,1}(\Gamma)$ satisfying

$$
\partial_{z}(\rho \mu)=0 \text {. }
$$

The complex vector space $\Omega^{-1,1}(\Gamma)$ is identified with the holomorphic tangent space to $\mathfrak{D}(\Gamma)$ at the origin. Choose a basis $\mu_{1}, \ldots, \mu_{d}$ for $\Omega^{-1,1}(\Gamma)$, set $\mu=t_{1} \mu_{1}+\cdots+t_{d} \mu_{d}$, where $t_{1}, \ldots, t_{d}$ are such that $\|\mu\|_{\infty}<1$, and let $f^{\mu}$ be the normalized solution of the Beltrami equation. Then the correspondence $\left(t_{1}, \ldots, t_{d}\right) \mapsto \Gamma^{\mu}=f^{\mu} \circ \Gamma \circ\left(f^{\mu}\right)^{-1}$ defines complex coordinates in a neighborhood of the origin in $\mathfrak{D}(\Gamma)$, called the Bers coordinates. The holomorphic cotangent space to $\mathfrak{D}(\Gamma)$ at the origin can be naturally identified with the vector space $\Omega^{2,0}(\Gamma)$ of holomorphic quadratic differentials — holomorphic functions $q$ on $\Omega$ satisfying

$$
q(\gamma z) \gamma^{\prime}(z)^{2}=q(z), \quad \gamma \in \Gamma .
$$

The pairing between holomorphic cotangent and tangent spaces to $\mathfrak{D}(\Gamma)$ at the origin is given by

$$
(q, \mu)=\iint_{F} q(z) \mu(z) d^{2} z .
$$

Corresponding anti-holomorphic cotangent space to $\mathfrak{D}(\Gamma)$ at $\Gamma$ is identified with the vector space $\Omega^{0,2}(\Gamma)=\overline{\Omega^{2,0}(\Gamma)}$ of anti-holomorphic quadratic differentials.

There is a natural isomorphism $\Phi^{\mu}$ between the deformation spaces $\mathfrak{D}(\Gamma)$ and $\mathfrak{D}\left(\Gamma^{\mu}\right)$, which maps $\Gamma^{\nu} \in \mathfrak{D}(\Gamma)$ to $\left(\Gamma^{\mu}\right)^{\lambda} \in \mathfrak{D}\left(\Gamma^{\mu}\right)$, where, in accordance with $f^{\nu}=f^{\lambda} \circ f^{\mu}$,

$$
\lambda=\left(\frac{\nu-\mu}{1-\nu \bar{\mu}} \frac{f_{z}^{\mu}}{\bar{f}_{\bar{z}}^{\mu}}\right) \circ\left(f^{\mu}\right)^{-1} .
$$

Isomorphism $\Phi^{\mu}$ allows us to identify the holomorphic tangent space to $\mathfrak{D}(\Gamma)$ at $\Gamma^{\mu}$ with the complex vector space $\Omega^{-1,1}\left(\Gamma^{\mu}\right)$, and holomorphic cotangent space to $\mathfrak{D}(\Gamma)$ at $\Gamma^{\mu}$ with the complex vector space $\Omega^{2,0}\left(\Gamma^{\mu}\right)$. It also allows us to introduce Bers coordinates in the neighborhood of $\Gamma^{\mu}$ in $\mathfrak{D}(\Gamma)$, and to show directly that these coordinates transform complex-analytically. For the de Rham differential $d$ on $\mathfrak{D}(\Gamma)$ we denote by $d=\partial+\bar{\partial}$ decomposition into $(1,0)$ and $(0,1)$ components.

The differential of isomorphism $\Phi^{\mu}: \mathfrak{D}(\Gamma) \simeq \mathfrak{D}\left(\Gamma^{\mu}\right)$ at $\nu=\mu$ is given by the linear map $D^{\mu}: \Omega^{-1,1}(\Gamma) \rightarrow \Omega^{-1,1}\left(\Gamma^{\mu}\right)$,

$$
\nu \mapsto D^{\mu} \nu=P_{-1,1}^{\mu}\left[\left(\frac{\nu}{1-|\mu|^{2}} \frac{f_{z}^{\mu}}{\bar{f}_{\bar{z}}^{\mu}}\right) \circ\left(f^{\mu}\right)^{-1}\right],
$$

where $P_{-1,1}^{\mu}$ is orthogonal projection from $\mathcal{H}^{-1,1}\left(\Gamma^{\mu}\right)$ to $\Omega^{-1,1}\left(\Gamma^{\mu}\right)$. The map $D^{\mu}$ allows us to extend a tangent vector $\nu$ at the origin of $\mathfrak{D}(\Gamma)$ to vector 
field $\frac{\partial}{\partial t_{\nu}}$ defined on the coordinate neighborhood of the origin,

$$
\left.\frac{\partial}{\partial t_{\nu}}\right|_{\Gamma^{\mu}}=D^{\mu} \nu \in \Omega^{-1,1}\left(\Gamma^{\mu}\right)
$$

The scalar product (4.1) in $\Omega^{-1,1}\left(\Gamma^{\mu}\right)$ defines a Hermitian metric on the deformation space $\mathfrak{D}(\Gamma)$. This metric is called the Weil-Petersson metric and it is Kähler. We denote its symplectic form by $\omega_{W P}$,

$$
\left.\omega_{W P}\left(\frac{\partial}{\partial t_{\mu}}, \frac{\partial}{\partial \bar{t}_{\nu}}\right)\right|_{\Gamma^{\lambda}}=\frac{i}{2}\left(D^{\lambda} \mu, D^{\lambda} \nu\right), \quad \mu, \nu \in \Omega^{-1,1}(\Gamma) .
$$

When $\Gamma$ is a Schottky group of marked compact Riemann surface $X$ of genus $g>1$, the deformation space $\mathfrak{D}(\Gamma)$ is the Schottky space $\mathfrak{S}_{g}$ of $X$. When $\Gamma$ is a Fuchsian group, such that components $\mathbb{U}$ and $\mathbb{L}$ cover, respectively, the marked compact Riemann surface $X$ of genus $g>1$ and its mirror image $\bar{X}$, the deformation space $\mathfrak{D}(\Gamma)$ contains the Teichmüller space $\mathfrak{T}_{g}$ of $X$ as a submanifold. Namely, the choice of the subspace of $\mathcal{B}^{-1,1}(\Gamma)$ consisting of $\mu$ with the reflection symmetry:

$$
\mu(\bar{z})=\overline{\mu(z)}, \quad z \in \mathbb{C},
$$

gives rise to the real-analytic embedding $\mathfrak{T}_{g} \hookrightarrow \mathfrak{D}(\Gamma)$. In this case, every group $\Gamma^{\mu}$ is a Fuchsian group. The choice of a subspace of $\mathcal{B}^{-1,1}(\Gamma)$ consisting of $\mu$ that are identically 0 on the lower half-plane $\mathbb{L}$, gives rise to the complex-analytic embedding $\mathfrak{T}_{g} \hookrightarrow \mathfrak{D}(\Gamma)$. In this case $\Gamma^{\mu}$ is, in general, a quasi-Fuchsian group. Its domain of discontinuity has two components $\Omega_{1}^{\mu}=f^{\mu}(\mathbb{U})$ and $\Omega_{2}^{\mu}=f^{\mu}(\mathbb{L})$, covering Riemann surfaces $X^{\mu} \simeq \Gamma^{\mu} \backslash \Omega_{1}^{\mu}$ and $\bar{X}$ respectively. The Weil-Petersson metric on $\mathfrak{D}(\Gamma)$ restricts to the Weil-Petersson metric of the Teichmüller space $\mathfrak{T}_{g}$. It is given by the same formula (4.1), where now $F$ is a fundamental domain for $\Gamma$ in $\Omega_{1}$. We continue to denote by $d=\partial+\bar{\partial}$ decomposition of de Rham differential $d$ on $\mathfrak{T}_{g}$ into $(1,0)$ and $(0,1)$ components. The Teichmüller space $\mathfrak{T}_{g}$ is the universal covering space for the moduli space $\mathfrak{M}_{g}$ of compact Riemann surfaces of genus $g>1$.

4.2. Formal geometry on deformation spaces. A formal function on a deformation space $\mathfrak{D}(\Gamma)$ is an element of $C^{\infty}(\mathfrak{D}(\Gamma))((\hbar))$ - a formal power series in $\hbar$ with coefficients in $C^{\infty}(\mathfrak{D}(\Gamma))$. Correspondingly, a formal 1-form on $\mathfrak{D}(\Gamma)$ is a formal power series in $\hbar$ with coefficients being 1-forms on $\mathfrak{D}(\Gamma)$. For a formal function $\mathcal{F}$ on $\mathfrak{D}(\Gamma), \partial \mathcal{F}$ and $\bar{\partial} \mathcal{F}$ are formal $(1,0)$ and $(0,1)$ forms on $\mathfrak{D}(\Gamma)$. For every $t \in \mathfrak{D}(\Gamma)$ there is an associated Riemann surface $X_{t} \simeq \Gamma_{t} \backslash \Omega_{t}$, and $\partial \mathcal{F}(t)$ and $\bar{\partial} \mathcal{F}(t)$ are represented by formal holomorphic and anti-holomorphic quadratic differentials for $\Gamma_{t}$. The Riemann surfaces $X_{t}$ form a holomorphic family parameterized by $\mathfrak{D}(\Gamma)$.

It follows from Definition 3.1 that the free energy $\mathcal{F}_{X}$ gives rise to a formal function $\mathcal{F}$ on the Schottky space $\mathfrak{S}_{g}$, or on the Teichmüller space $\mathfrak{T}_{g}$, depending on the choice of a global coordinate on $X$. Namely, to every point $t \in \mathfrak{S}_{g}$ (or $t \in \mathfrak{T}_{g}$ ) there is an associated Riemann surface $X_{t}$ with 
Schottky (or quasi-Fuchsian) global coordinate, and $\mathcal{F}(t)=\mathcal{F}_{X_{t}} \in \frac{1}{\hbar} \mathbb{C}[[\hbar]]$. As it was pointed out in Remark 3.3 , actually $\mathcal{F}-\frac{1}{2 \pi \hbar} S_{c l}$ is a formal function on the moduli space $\mathfrak{M}_{g}$.

It also follows from Definition 3.5 that for every $t \in \mathfrak{S}_{g}$ (or $t \in \mathfrak{S}_{g}$ ) onepoint correlation functions $\left\langle\left\langle T(z) X_{t}\right\rangle\right\rangle$ and $\left\langle\left\langle\bar{T}(\bar{z}) X_{t}\right\rangle\right\rangle$ are formal quadratic differentials for $\Gamma_{t}$ in $z$ and $\bar{z}$. We will show in Section 6 that they are holomorphic and anti-holomorphic formal quadratic differentials that represent (up to an additional one-loop term) formal $(1,0)$ and $(0,1)$-forms $\partial \mathcal{F}$ and $\bar{\partial} \mathcal{F}$, where $\mathcal{F}$ is a formal function associated with free energy. Multi-point correlation functions admit similar interpretation. In Sections 7 and 8 we present all details for cases $\left\langle\left\langle T(z) T(w) X_{t}\right\rangle\right\rangle$ and $\left\langle\left\langle T(z) \bar{T}(\bar{w}) X_{t}\right\rangle\right\rangle$.

4.3. Variational formulas. Here we collect variational formulas needed in the next section. For $\mu \in \mathcal{A}^{-1,1}(\Gamma)$ quasiconformal mappings $f^{\varepsilon \mu}$ depend holomorphically on $\varepsilon$ in some neighborhood of $0 \in \mathbb{C}$. Setting

$$
\dot{f}=\left.\frac{d}{d \varepsilon}\right|_{\varepsilon=0} f^{\varepsilon \mu},
$$

we obtain

$$
\dot{f}(z)=-\frac{1}{\pi} \iint_{\mathbb{C}} \frac{z(z-1) \mu(w)}{(w-z) w(w-1)} d^{2} w \quad \text { and } \quad \dot{f}_{\bar{z}}=\mu .
$$

A tensor of type $(l, m)$ for $\Gamma$, where $l$ and $m$ are integers, is a $C^{\infty}$-function $\theta$ on $\Omega$ satisfying

$$
\theta \circ \gamma\left(\gamma^{\prime}\right)^{l}\left(\overline{\gamma^{\prime}}\right)^{m}=\theta, \quad \gamma \in \Gamma
$$

Let $\theta^{\varepsilon}$ be a smooth family of tensors of type $(l, m)$ for the holomorphic family $\Gamma^{\varepsilon \mu}$, where it is always assumed that $\mu \in \Omega^{-1,1}(\Gamma)$, and $\varepsilon \in \mathbb{C}$ is sufficiently small. Set

$$
\left(f^{\varepsilon \mu}\right)^{*}\left(\theta^{\varepsilon}\right)=\theta^{\varepsilon} \circ f^{\varepsilon \mu}\left(f_{z}^{\varepsilon \mu}\right)^{l}\left(\bar{f}_{\bar{z}}^{\varepsilon \mu}\right)^{m},
$$

which is a tensor of type $(l, m)$ for $\Gamma$ - a pull-back of the tensor $\theta^{\varepsilon}$ for $\Gamma^{\varepsilon \mu}$ by $f^{\varepsilon \mu}$. The Lie derivatives of the family $\theta^{\varepsilon}$ along the vector fields $\frac{\partial}{\partial t_{\mu}}$ and $\frac{\partial}{\partial t_{\mu}}$ are defined in the standard way,

$$
L_{\mu} \theta=\left.\frac{\partial}{\partial \varepsilon}\right|_{\varepsilon=0}\left(f^{\varepsilon \mu}\right)^{*}\left(\theta^{\varepsilon}\right) \quad \text { and } \quad L_{\bar{\mu}} \theta=\left.\frac{\partial}{\partial \bar{\varepsilon}}\right|_{\varepsilon=0}\left(f^{\varepsilon \mu}\right)^{*}\left(\theta^{\varepsilon}\right) .
$$

When $\theta$ is a function on $\mathfrak{D}(\Gamma)$ - a tensor of type $(0,0)$, Lie derivatives reduce to directional derivatives $L_{\mu} \theta=(\partial \theta)(\mu)$ and $L_{\bar{\mu}} \theta=(\bar{\partial} \theta)(\bar{\mu})$.

When $\theta$ is a $(1,0)$-form on $\mathfrak{D}(\Gamma)$, i.e., a family $\theta^{\varepsilon}$ of holomorphic quadratic differentials for $\Gamma^{\varepsilon \mu}$, we have

$$
\partial \theta=\sum_{i=1}^{d} d t_{i} \wedge L_{\mu_{i}} \theta \quad \text { and } \quad \bar{\partial} \theta=\sum_{i=1}^{d} d \bar{t}_{i} \wedge L_{\bar{\mu}_{i}} \theta,
$$

where $d t_{1}, \ldots, d t_{d}$ is the basis for $\Omega^{2,0}(\Gamma)$ dual to the basis $\mu_{1}, \ldots, \mu_{d}$ for $\Omega^{-1,1}(\Gamma)$. 
Let $\mathcal{H}^{2,0}(\Gamma)$ be the Hilbert space of quadratic differentials for $\Gamma$ with the inner product

$$
\left(q_{1}, q_{2}\right)=\iint_{F} q_{1}(z) \overline{q_{2}(z)} \rho(z)^{-1} d^{2} z,
$$

where $F$ is a fundamental domain for $\Gamma$ in $\Omega$, and let $P: \mathcal{H}^{2,0}(\Gamma) \rightarrow \Omega^{2,0}(\Gamma)$ be the orthogonal projection onto the subspace of holomorphic quadratic differentials. It immediately follows from Stokes' theorem that the quadratic differential $\mathcal{D}_{z} h$, where $h$ is a smooth $\Gamma$-automorphic function on $\Omega$, is orthogonal to $\Omega^{2,0}(\Gamma)$.

Now suppose that for a $(1,0)$-form $\theta$ on $\mathfrak{D}(\Gamma)$,

$$
L_{\mu} \theta(z)=\iint_{F} Q(z, w) \mu(w) d^{2} w
$$

where $Q(z, w)$ is a smooth quadratic differential for $\Gamma$ in $z$ and $w$. Identification of holomorphic tangent and cotangent spaces to $\mathfrak{D}(\Gamma)$ with $\Omega^{-1,1}(\Gamma)$ and $\Omega^{2,0}(\Gamma)$ in Section 4.1, allows us to identify the $(2,0)$-form $\partial \theta$ on $\mathfrak{D}(\Gamma)$ at the point $\Gamma$ with $P_{w} P_{z} Q(w, z)-P_{z} P_{w} Q(z, w)$ - the holomorphic quadratic differential for $\Gamma$ in $z$ and $w$, where $P_{z}$ and $P_{w}$ are corresponding projection operators with respect to $z$ and $w$. Explicitly,

$$
\partial \theta\left(\frac{\partial}{\partial t_{i}}, \frac{\partial}{\partial t_{j}}\right)=-\iint_{F} \iint_{F} Q(z, w)\left(\mu_{i}(z) \mu_{j}(w)-\mu_{j}(z) \mu_{i}(w)\right) d^{2} z d^{2} w .
$$

The symmetric part $\frac{1}{2}\left(P_{z} P_{w} Q(z, w)+P_{w} P_{z} Q(w, z)\right)$ corresponds to the following 2-tensor

$$
\partial_{s} \theta=\sum_{i=1}^{d} L_{\mu_{i}} \theta \otimes_{s} d t_{i}
$$

defined in a coordinate chart of the origin in $\mathfrak{D}(\Gamma)$, where $\otimes_{s}$ stands for the symmetrized tensor product, $d t_{i} \otimes_{s} d t_{j}=\frac{1}{2}\left(d t_{i} \otimes d t_{j}+d t_{j} \otimes d t_{i}\right)$. Explicitly,

$$
\partial_{s} \theta\left(\frac{\partial}{\partial t_{i}}, \frac{\partial}{\partial t_{j}}\right)=\frac{1}{2} \iint_{F} \iint_{F} Q(z, w)\left(\mu_{i}(z) \mu_{j}(w)+\mu_{j}(z) \mu_{i}(w)\right) d^{2} z d^{2} w .
$$

If $S$ is a function on $\mathfrak{D}(\Gamma)$, then, by definition, $\partial_{s} S=\partial S$, and we have

$$
\partial_{s}\left(\partial_{s} S\right)=\sum_{i, j=1}^{d} \frac{\partial^{2} S}{\partial t_{i} \partial t_{j}} d t_{i} \otimes_{s} d t_{j}
$$

while $\partial(\partial S)=0$. In general, $\partial_{s}$ can be extended to a linear operator mapping $(n, 0)$-symmetric tensors on a coordinate chart of the origin in $\mathfrak{D}(\Gamma)$ to symmetric $(n+1,0)$-tensors.

For the Lie derivatives of vector fields $\nu^{\epsilon \mu}=D^{\epsilon \mu} \nu$ we get Wol86]

$$
L_{\mu} \nu=0 \quad \text { and } \quad L_{\bar{\mu}} \nu=-\bar{\partial} \rho^{-1} \bar{\partial}\left(\Delta_{0}+\frac{1}{2}\right)^{-1}(\mu \bar{\nu}) .
$$


For every $\Gamma^{\mu} \in \mathfrak{D}(\Gamma)$, the density $\rho^{\mu}$ of the hyperbolic metric on $\Omega^{\mu}$ is a $(1,1)$-tensor for $\Gamma^{\mu}$. Lie derivatives of the smooth family of $(1,1)$-tensors $\rho$ parameterized by $\mathfrak{D}(\Gamma)$ are given by the following formulas:

$$
\begin{aligned}
L_{\mu} \rho & =L_{\bar{\mu}} \rho=0, \\
L_{\mu} L_{\bar{\nu}} \rho & =\frac{1}{2} \rho\left(\Delta_{0}+\frac{1}{2}\right)^{-1}(\mu \bar{\nu}),
\end{aligned}
$$

belonging, correspondingly, to Ahlfors [Ahl61] and Wolpert [Wol86]. Since $f^{\varepsilon \mu}$ depends holomorphically on $\varepsilon$, we get from (4.7)

$$
\left.\frac{\partial}{\partial \varepsilon}\right|_{\varepsilon=0}\left(\varphi_{c l}^{\varepsilon \mu} \circ f^{\varepsilon \mu}\right)=-\dot{f}_{z} .
$$

For every $\Gamma^{\mu} \in \mathfrak{D}(\Gamma)$ let $\Omega_{1}^{\mu}$ be the component of domain of discontinuity of $\Gamma^{\mu}$ such that $X^{\mu} \simeq \Gamma^{\mu} \backslash \Omega_{1}^{\mu}$, and let $J_{\mu}: \mathbb{U} \rightarrow \Omega_{1}^{\mu}$ be the corresponding covering map. The assignment $\vartheta(\mu)=\mathcal{S}\left(J_{\mu}^{-1}\right) \in \Omega^{2,0}\left(\Gamma^{\mu}\right)$ defines a $(1,0)$ form on $\mathfrak{D}(\Gamma)$. It was proved in [ZT87c] for the case $\mathfrak{D}(\Gamma)=\mathfrak{S}_{g}$, and in TT03a for the case $\mathfrak{D}(\Gamma)=\mathfrak{T}_{g}$, that

$$
\partial S_{c l}=2 \vartheta
$$

as well as

$$
\begin{aligned}
& L_{\mu} \vartheta(z)=\frac{6}{\pi} \iint_{\mathbb{C}} \frac{\mu(w)}{(z-w)^{4}} d^{2} w, \\
& L_{\bar{\mu}} \vartheta(z)=-\frac{1}{2} \rho \overline{\mu(z) .}
\end{aligned}
$$

The integral in (4.11) is understood in the principal value sense, i.e., as a limit

$$
\lim _{\varepsilon \rightarrow 0} \iint_{|w-z| \geq \varepsilon} \frac{\mu(w)}{(z-w)^{4}} d^{2} w
$$

which exists for harmonic $\mu$. Formula (4.12) is equivalent to

$$
\bar{\partial} \partial S_{c l}=-2 i \omega_{W P}
$$

whereas interpretation of (4.11) in terms of $\partial_{s}^{2} S_{c l}$ will be given in Section 7.1 .

Remark 4.1. When $\Gamma$ is a Fuchsian group and $\left.\mu\right|_{\mathbb{L}}=0$, then according to [TT03b, Lemma 2.12] $L_{\mu} \vartheta(z)=0$.

Lie derivatives of a family of linear operators $\mathcal{O}^{\varepsilon}$ mapping tensors of type $(l, m)$ to tensors of type $\left(l^{\prime}, m^{\prime}\right)$ are defined by the formulas

$$
\begin{aligned}
& L_{\mu} \mathcal{O}=\left.\frac{\partial}{\partial \varepsilon}\right|_{\varepsilon=0}\left(f^{\varepsilon \mu}\right)^{*} \mathcal{O}^{\varepsilon}\left(\left(f^{\varepsilon \mu}\right)^{*}\right)^{-1}, \\
& L_{\bar{\mu}} \mathcal{O}=\left.\frac{\partial}{\partial \bar{\varepsilon}}\right|_{\varepsilon=0}\left(f^{\varepsilon \mu}\right)^{*} \mathcal{O}^{\varepsilon}\left(\left(f^{\varepsilon \mu}\right)^{*}\right)^{-1},
\end{aligned}
$$


and satisfy

$$
L_{\mu}(\mathcal{O}(\theta))=L_{\mu} \mathcal{O}(\theta)+\mathcal{O}\left(L_{\mu} \theta\right), \quad L_{\bar{\mu}}(\mathcal{O}(\theta))=L_{\bar{\mu}} \mathcal{O}(\theta)+\mathcal{O}\left(L_{\bar{\mu}} \theta\right) .
$$

For the families of $\bar{\partial}$-operators mapping $(n, 0)$-tensors to $(n, 1)$-tensors, and of $\partial$-operators mapping $(0,1)$-tensors to $(1,1)$-tensors, we have the following formulas,

$$
\begin{array}{llrl}
L_{\mu} \bar{\partial} & =-\mu \rho^{n} \partial \rho^{-n}, & & L_{\bar{\mu}} \bar{\partial}=0 \\
L_{\mu} \partial=0, & L_{\bar{\mu}} \partial=-\bar{\partial} \bar{\mu} .
\end{array}
$$

Hence for the operator $\Delta_{0}+\frac{1}{2}=-\rho^{-1} \partial \bar{\partial}+\frac{1}{2}$ we get

$$
L_{\mu}\left(\Delta_{0}+\frac{1}{2}\right)=\rho^{-1} \partial \mu \partial, \quad L_{\bar{\mu}}\left(\Delta_{0}+\frac{1}{2}\right)=\rho^{-1} \bar{\partial} \bar{\mu} \bar{\partial} .
$$

The Lie derivatives of operators $\partial$ and $\mathcal{D}_{z}=\rho \partial \rho^{-1} \partial$ as operators from functions to $(1,0)$ and $(2,0)$ tensors respectively, are given by

$$
\begin{array}{ll}
L_{\mu} \partial=0, & L_{\mu} \mathcal{D}_{z}=0, \\
L_{\bar{\mu}} \partial=-\bar{\mu} \bar{\partial}, & L_{\bar{\mu}} \mathcal{D}_{z}=-\bar{\mu} \bar{\partial} \partial-\rho \partial \rho^{-1} \bar{\mu} \bar{\partial} .
\end{array}
$$

It follows from (4.14) that for a family $\theta^{\varepsilon}(z)$ of holomorphic quadratic differentials $L_{\bar{\mu}} \theta(z)$ is holomorphic in $z$, whereas $L_{\mu} \theta(z)$, in general, is not. Thus if

$$
L_{\bar{\mu}} \theta(z)=\iint_{F} \tilde{Q}(z, w) \overline{\mu(w)} d^{2} w
$$

where $\tilde{Q}(z, w)$ is a smooth quadratic differential for $\Gamma$ in $z$ and $\bar{w}$, then the $(1,1)$-form $\bar{\partial} \theta$ on $\mathfrak{D}(\Gamma)$ at the point $\Gamma$ is identified with the quadratic differential $-P_{\bar{w}} \tilde{Q}(z, w)$ for $\Gamma$, which is holomorphic in $z$ and anti-holomorphic in w. Explicitly,

$$
\bar{\partial} \theta\left(\frac{\partial}{\partial t_{i}}, \frac{\partial}{\partial \bar{t}_{j}}\right)=-\iint_{F} \iint_{F} \tilde{Q}(z, w) \mu_{i}(z) \overline{\mu_{j}(w)} d^{2} z d^{2} w .
$$

\section{THE PROPAGATOR}

The propagator $G(P, Q)$ of quantum Liouville theory - the integral kernel of the operator $G=\frac{1}{2}\left(\Delta_{0}+\frac{1}{2}\right)^{-1}$, is uniquely characterized by the following properties.

P1. $G$ is a smooth function on $X \times X \backslash \mathrm{D}$, where $\mathrm{D}$ is the diagonal $P=Q$ in $X \times X$.

P2. $G$ is symmetric, $G(P, Q)=G(Q, P)$ for $P, Q \in X$.

P3. For fixed $Q \in X, G(P, Q)$ as a function of $P \in X \backslash\{Q\}$ satisfies

$$
\left(\Delta_{0}+\frac{1}{2}\right) G=0 .
$$

P4. For fixed $Q \in X$, the function

$$
G(P, Q)+\frac{1}{2 \pi} \log |z(P)-z(Q)|^{2}
$$


is continuous in some neighborhood $U$ of $Q$, where $z$ is a local coordinate in $U$.

It follows from these properties that for every $g \in C^{\infty}(X)$ the function

$$
h(P)=\iint_{X} G(P, Q) g(Q) d Q
$$

satisfies the equation

$$
\left(\Delta_{0}+\frac{1}{2}\right) h=\frac{1}{2} g
$$

In particular,

$$
\iint_{X} G(P, Q) d Q=1 .
$$

On the upper half-plane $\mathbb{U}$, the kernel for the integral operator $\frac{1}{2}\left(\Delta_{0}+\frac{1}{2}\right)^{-1}$ is given by (see, e.g., Tak96a])

$$
\mathcal{G}(z, w)=\frac{1}{2 \pi} \int_{0}^{1} \frac{t(1-t)}{(t+u)^{2}} d t=\frac{2 u+1}{2 \pi} \log \frac{u+1}{u}-\frac{1}{\pi},
$$

where

$$
u(z, w)=\frac{|z-w|^{2}}{4 \operatorname{Im} z \operatorname{Im} w} .
$$

The function $\mathcal{G}$ has the property

$$
\mathcal{G}(\sigma z, \sigma w)=\mathcal{G}(z, w), \quad \sigma \in \operatorname{PSL}(2, \mathbb{R}) .
$$

It terms of the Fuchsian global coordinate $z$ with the covering map $J_{F}: \mathbb{U} \rightarrow$ $X$, the propagator $G_{F}(z, w)=G\left(J_{F}(z), J_{F}(w)\right)$ is given by the method of images,

$$
G_{F}(z, w)=\sum_{\gamma \in \Gamma} \mathcal{G}(z, \gamma w)
$$

and it follows from (5.5) that $G_{F}(z, w)$ is $\Gamma$-automorphic in $z$ and $w$,

$$
G_{F}\left(\gamma_{1} z, \gamma_{2} w\right)=G_{F}(z, w), \quad \gamma_{1}, \gamma_{2} \in \Gamma .
$$

If $z_{K}$ is another global coordinate on $X$ with the covering map $J_{K}: \Omega_{K} \rightarrow$ $X$, then $G_{K}\left(z_{K}, w_{K}\right)=G\left(J_{K}\left(z_{K}\right), J_{K}\left(w_{K}\right)\right)$ satisfies

$$
G_{K}\left(z_{K}, w_{K}\right)=G_{F}\left(J^{-1}\left(z_{K}\right), J^{-1}\left(w_{K}\right)\right),
$$

where $J=J_{K}^{-1} \circ J_{F}$. 
5.1. Behavior near diagonal and explicit formulas. Here we present basic properties of the propagator of quantum Liouville theory. It is convenient to use the Fuchsian global coordinate $z$ on $X$ and to write $G=G_{F}$.

It follows from (5.3)-(5.4) that

$$
\mathcal{G}(z, w)=-\frac{1}{2 \pi} \log \frac{|z-w|^{2}}{\operatorname{Im} z \operatorname{Im} w}-\frac{1-\log 2}{\pi}+o(1) \quad \text { as } \quad w \rightarrow z,
$$

and, therefore,

$$
G(z, w)=-\frac{1}{2 \pi} \log |z-w|^{2}+O(1) \quad \text { as } \quad w \rightarrow z .
$$

Similarly, as $w \rightarrow z$,

$$
\begin{aligned}
\partial_{z} \mathcal{G}(z, w) & =-\frac{1}{2 \pi} \frac{w-\bar{z}}{(z-w)(z-\bar{z})}+o(1) \\
\partial_{z} \partial_{w} \mathcal{G}(z, w) & =-\frac{1}{2 \pi} \frac{1}{(z-w)^{2}}-\frac{1}{\pi} \frac{\bar{z}-\bar{w}}{z-w} \frac{1}{(z-\bar{z})^{2}}+o(|z-w|), \\
\partial_{z} \partial_{\bar{w}} \mathcal{G}(z, w) & =-\frac{1}{\pi} \frac{1}{(z-\bar{z})^{2}} \log |z-w|^{2}+O(1),
\end{aligned}
$$

so that

$$
\begin{aligned}
\partial_{z} G(z, w) & =-\frac{1}{2 \pi(z-w)}+O(1), \\
\partial_{z} \partial_{w} G(z, w) & =-\frac{1}{2 \pi(z-w)^{2}}-\frac{1}{\pi} \frac{\bar{z}-\bar{w}}{z-w} \frac{1}{(z-\bar{z})^{2}}+O(1), \\
\partial_{z} \partial_{\bar{w}} G(z, w) & =-\frac{1}{\pi(z-\bar{z})^{2}} \log |z-w|^{2}+O(1) .
\end{aligned}
$$

In terms of the Fuchsian global coordinate, the regularization (3.8) at the coincident points is given by

$$
G(z, z)=\sum_{\substack{\gamma \in \Gamma \\ \gamma \neq \text { id }}} \mathcal{G}(z, \gamma z)-\frac{1-\log 2}{\pi}
$$

(see Tak93]). It follows from (5.5) that $G(z, z)$ is $\Gamma$-automorphic,

$$
G(\gamma z, \gamma z)=G(z, z), \quad \gamma \in \Gamma .
$$

Similarly, the regularization (3.12) of the tadpole graph $H(z)=\partial_{z} \partial_{z} G(z, z)$ is given by

$$
H(z)=\left.\sum_{\substack{\gamma \in \Gamma \\ \gamma \neq \text { id }}} \partial_{z} \partial_{w} \mathcal{G}(z, \gamma w)\right|_{w=z}
$$

(see [Tak94, Tak96a]). It follows from (5.5) that $H$ is a quadratic differential for $\Gamma$, and

$$
\partial_{z} H(z)=\left.2 \sum_{\substack{\gamma \in \Gamma \\ \gamma \neq \mathrm{id}}} \partial_{z}^{2} \partial_{w} \mathcal{G}(z, \gamma w)\right|_{w=z}
$$


Using property P3, we also obtain

$$
\partial_{\bar{z}} H(z)=\frac{1}{2} \rho(z) \partial_{z} G(z, z) .
$$

It follows from (5.8) that $G(z, z)$ gives rise to a well-defined smooth function $G(P, P)$ on $X$. However, it follows from (3.12) and the classical formula

$$
\frac{f^{\prime}(z) f^{\prime}(w)}{(f(z)-f(w))^{2}}=\frac{1}{(z-w)^{2}}+\frac{1}{6} \mathcal{S}(f)(z)+\frac{1}{12} \mathcal{S}(f)^{\prime}(z)(w-z)+\ldots
$$

as $w \rightarrow z$, where dots stand for $O\left(|z-w|^{2}\right)$ term, that

$$
H_{K}\left(z_{K}\right)=H\left(J^{-1}\left(z_{K}\right)\right)\left(J^{-1}\right)^{\prime}\left(z_{K}\right)^{2}-\frac{1}{12 \pi} \mathcal{S}\left(J^{-1}\right)\left(z_{K}\right) .
$$

Hence $H(z)$, in accordance with [Tak94, Tak96a], behaves like " $-1 / 12 \pi$ of a projective connection" under changes of global coordinates.

The following explicit formulas

$$
\mathcal{D}_{z} \mathcal{G}(z, w)=\frac{1}{2 \pi} \frac{(w-\bar{w})^{2}}{(z-w)^{2}(z-\bar{w})^{2}}
$$

and

$$
\begin{array}{rlrl}
\mathcal{D}_{z} \partial_{w} \mathcal{G}(z, w) & =\frac{1}{\pi} \frac{w-\bar{w}}{(z-w)^{3}(z-\bar{w})}, & \mathcal{D}_{z} \mathcal{D}_{w} \mathcal{G}(z, w) & =\frac{3}{\pi} \frac{1}{(z-w)^{4}} \\
\mathcal{D}_{z} \partial_{\bar{w}} \mathcal{G}(z, w)=\frac{1}{\pi} \frac{\bar{w}-w}{(z-w)(z-\bar{w})^{3}}, & \mathcal{D}_{z} \mathcal{D}_{\bar{w}} \mathcal{G}(z, w)=\frac{3}{\pi} \frac{1}{(z-\bar{w})^{4}}
\end{array}
$$

give the following asymptotic formulas as $w \rightarrow z$ :

$$
\begin{aligned}
\mathcal{D}_{z} G(z, w) & =\frac{1}{2 \pi} \frac{(w-\bar{w})^{2}}{(z-w)^{2}(z-\bar{w})^{2}}+O(1), \\
\mathcal{D}_{z} \partial_{w} G(z, w) & =\frac{1}{\pi} \frac{w-\bar{w}}{(z-w)^{3}(z-\bar{w})}+O(1), \\
\mathcal{D}_{z} \partial_{\bar{w}} G(z, w) & =\frac{1}{\pi} \frac{\bar{w}-w}{(z-w)(z-\bar{w})^{3}}+O(1), \\
\mathcal{D}_{z} \mathcal{D}_{w} G(z, w) & =\frac{3}{\pi} \frac{1}{(z-w)^{4}}+O(1),
\end{aligned}
$$

and explicit formulas

$$
\begin{aligned}
& \mathcal{D}_{z} \mathcal{D}_{w} G(z, w)=\frac{3}{\pi} \sum_{\gamma \in \Gamma} \frac{\gamma^{\prime}(w)^{2}}{(z-\gamma w)^{4}} \\
& \mathcal{D}_{z} \mathcal{D}_{\bar{w}} G(z, w)=\frac{3}{\pi} \sum_{\gamma \in \Gamma} \frac{\gamma^{\prime}(\bar{w})^{2}}{(z-\gamma \bar{w})^{4}} .
\end{aligned}
$$

Since

$$
\mathbb{U}=\bigcup_{\gamma \in \Gamma} \gamma F
$$


where $F$ is a fundamental domain for $\Gamma$ in $\mathbb{U}$, we obtain from (5.25) and the Ahlfors' classical reproducing formula in [Ahl87, Ch. VI.D., Lemma 2],

$$
(P q)(z)=4 \iint_{F} \mathcal{D}_{z} \mathcal{D}_{\bar{w}} G(z, w) q(w) \rho(w)^{-1} d^{2} w, \quad q \in \mathcal{H}^{2,0}(\Gamma),
$$

so that $P(z, w)=4 \mathcal{D}_{z} \mathcal{D}_{\bar{w}} G(z, w)$ is an integral kernel of the projection operator $P: \mathcal{H}^{2,0}(\Gamma) \rightarrow \Omega^{2,0}(\Gamma)$. Representation (5.27) was used in [Tak94, Tak96a, and plays a fundamental role in this paper.

In general, $\mathcal{D}_{z} \mathcal{D}_{\bar{w}} G(z, w)$, where $z$ and $w$ are local coordinates on $X$, is a holomorphic quadratic differential on $X$ with respect to the first variable, and is an anti-holomorphic quadratic differential on $X$ with respect to the second variable. The expression $P(z, w)=4 \mathcal{D}_{z} \mathcal{D}_{\bar{w}} G(z, w)$ is an integral kernel of the orthogonal projection operator $P: \mathcal{H}^{2,0}(X) \rightarrow \Omega^{2,0}(X)$, where the inner product in $\mathcal{H}^{2,0}(X)$ is defined by using the hyperbolic metric on $X$.

Similarly, $\mathcal{D}_{z} \mathcal{D}_{w} G(z, w)$ is a meromorphic quadratic differential on $X$ in variables $z$ and $w$, with the fourth order pole at $z=w$. It behaves like a quadratic differential in $z$ and $w$ under a change of global coordinates. Let $z$ be a global coordinate on $X$. Using (5.19) we get as $w \rightarrow z$,

$$
\mathcal{D}_{z} \mathcal{D}_{w} G(z, w)=\frac{3}{\pi}\left(\frac{1}{(z-w)^{4}}+\frac{\mathcal{S}\left(J^{-1}\right)(z)}{3(z-w)^{2}}-\frac{\mathcal{S}\left(J^{-1}\right)^{\prime}(z)}{6(z-w)}\right)+O(1) .
$$

Finally,

$$
R(z, w)=4 \rho(z)^{-1} \partial_{\bar{z}} \mathcal{D}_{w} G(z, w)
$$

is a meromorphic quadratic differential on $X$ in $w$ with a single simple pole at $w=z$, and is a $(-1,0)$-tensor with respect to $z$. We have

$$
R(z, w)=-\frac{1}{\pi(z-w)}+O(1) \quad \text { as } \quad w \rightarrow z
$$

and this expansion does not depend on the choice of local coordinates $z$ and $w$ in the neighborhood of the diagonal in $X \times X$. It follows from property $\mathbf{P 3}$ that for any choice of global coordinate $z$ on $X$, the kernel $R(z, w)$ satisfies the equation

$$
\partial_{z} R(z, w)+\left(\partial_{z} \varphi_{c l}\right)(z) R(z, w)=2 \mathcal{D}_{w} G(z, w),
$$

which implies

$$
\partial_{z}^{3} R(z, w)=2 \mathcal{D}_{z} \mathcal{D}_{w} G(z, w)-\left(2 \partial_{z} R(z, w)+R(z, w) \partial_{z}\right) \mathcal{S}\left(J^{-1}\right)(z)
$$

and

$$
\begin{gathered}
\mathcal{D}_{z}\left(R(z, w) \partial_{z} G(z, v)\right) \\
=2 \mathcal{D}_{w} \partial_{z} G(z, w) \partial_{z} G(z, v)+\left(2 \partial_{z} R(z, w)+R(z, w) \partial_{z}\right) \mathcal{D}_{z} G(z, v) .
\end{gathered}
$$


Remark 5.1. The kernel $R(z, w)$ is a Green's function of $\bar{\partial}$-operator acting on $(-1,0)$-tensors on $X-$ an integral kernel of the inverse operator $\bar{\partial}^{-1}$ on the space of trivial Beltrami differentials on $X$, which is an orthogonal complement in $\mathcal{H}^{-1,1}(X)$ to the subspace of harmonic Beltrami differentials. It is also used in the formulation of conformal Ward identities on Riemann surfaces in [EO87].

Remark 5.2. Operator $P$ can be also defined on the vector space of quadratic differentials for $\Gamma$ which are smooth everywhere on $F$ except at $z=w$, where they have the following asymptotic behavior:

$$
q(z)=\frac{a_{1}}{(z-w)^{4}}+\frac{a_{2}}{(z-w)^{2}}+\frac{a_{3}}{z-w}+O(1) \quad \text { and } \quad \partial_{\bar{z}} q(z)=O(1)
$$

as $z \rightarrow w$. The integral in (5.27) is understood in the principal value sense, and it follows from the Stokes' theorem, (5.28), and (5.30) $\Omega^{2,0}(\Gamma)$.

Remark 5.3. For a family of holomorphic quadratic differentials $\theta^{\varepsilon \mu}(z)$ for $\Gamma^{\varepsilon \mu}$ we have

Suppose that

$$
\begin{aligned}
\left(L_{\mu} \theta\right)(z)= & \left.\frac{\partial}{\partial \varepsilon}\right|_{\varepsilon=0}\left(\theta^{\varepsilon \mu} \circ f^{\varepsilon \mu}\left(f_{z}^{\varepsilon \mu}\right)^{2}\right)(z)=\left.\frac{\partial}{\partial \varepsilon}\right|_{\varepsilon=0} \theta^{\varepsilon \mu}(z) \\
& +\left.\left(\partial_{z} \theta\right)(z) \frac{\partial}{\partial \varepsilon}\right|_{\varepsilon=0} f^{\varepsilon \mu}(z)+\left.2 \theta(z) \frac{\partial}{\partial \varepsilon}\right|_{\varepsilon=0} f_{z}^{\varepsilon \mu}(z) .
\end{aligned}
$$

$$
\left.\frac{\partial}{\partial \varepsilon}\right|_{\varepsilon=0} \theta^{\varepsilon \mu}(z)=\iint_{F} Q_{1}(z, w) \mu(w) d^{2} w,
$$

where $Q_{1}(z, w)$ is a quadratic differential in $w$. Then by (4.3)

$$
\left(L_{\mu} \theta\right)(z)=\iint_{F} Q(z, w) \mu(w) d^{2} w
$$

where

$$
Q(z, w)=Q_{1}(z, w)+\left(2 \partial_{z} \mathcal{R}(z, w)+\mathcal{R}(z, w) \partial_{z}\right) \theta(z)
$$

and

$$
\mathcal{R}(z, w)=-\frac{1}{\pi} \sum_{\gamma \in \Gamma} \frac{z(z-1) \gamma^{\prime}(w)^{2}}{(\gamma w-z) \gamma w(\gamma w-1)}
$$

is a meromorphic quadratic differential for $\Gamma$ in $w$. By the Stokes' theorem, it is easy to prove that

$$
P_{w} \mathcal{R}(z, w)=\mathcal{R}(z, w)+R(z, w) .
$$

Thus we obtain

$$
\begin{array}{r}
P_{w}\left(Q_{1}\right)(z, w)+\left(2 \partial_{z} \mathcal{R}(z, w)+\mathcal{R}(z, w) \partial_{z}\right) \theta(z) \\
=P_{w}(Q)(z, w)-\left(2 \partial_{z} R(z, w)+R(z, w) \partial_{z}\right) \theta(z),
\end{array}
$$


where $P_{w}\left(Q_{1}\right)(z, w)$ is holomorphic in $z$.

5.2. Variational formulas. Here we collect variational formulas for the propagator $G$, which are necessary for Sections 68 .

Lemma 5.4. Let $z$ be a global coordinate on $X \simeq \Gamma \backslash \Omega$, and $\mu \in \Omega^{-1,1}(\Gamma)$. We have the following formulas, where $F$ is a fundamental domain for $\Gamma$ in $\Omega$.

(i) For $z \neq w$,

$$
\begin{aligned}
& L_{\mu} G(z, w)=2 \iint_{F} \partial_{v} G(z, v) \partial_{v} G(v, w) \mu(v) d^{2} v, \\
& L_{\bar{\mu}} G(z, w)=2 \iint_{F} \partial_{\bar{v}} G(z, v) \partial_{\bar{v}} G(v, w) \overline{\mu(v)} d^{2} v .
\end{aligned}
$$

(ii)

$$
\begin{aligned}
& L_{\mu} G(z, z)=2 \iint_{F}\left(\partial_{v} G(z, v)\right)^{2} \mu(v) d^{2} v, \\
& L_{\bar{\mu}} G(z, z)=2 \iint_{F}\left(\partial_{\bar{v}} G(z, v)\right)^{2} \overline{\mu(v)} d^{2} v .
\end{aligned}
$$

(iii) For $z \neq w$,

$$
\begin{aligned}
& L_{\mu} \partial_{z} G(z, w)=2 \iint_{F} \partial_{z} \partial_{v} G(z, v) \partial_{v} G(v, w) \mu(v) d^{2} v \\
& L_{\bar{\mu}} \partial_{z} G(z, w)=2 \iint_{F} \partial_{z} \partial_{\bar{v}} G(z, v) \partial_{\bar{v}} G(v, w) \overline{\mu(v)} d^{2} v .
\end{aligned}
$$

(iv) For $z \neq w$,

$$
\begin{aligned}
L_{\mu} \mathcal{D}_{z} G(z, w)= & 2 \iint_{F} \mathcal{D}_{z} \partial_{v} G(z, v) \partial_{v} G(v, w) \mu(v) d^{2} v \\
L_{\bar{\mu}} \mathcal{D}_{z} G(z, w)= & 2 \iint_{F} \mathcal{D}_{z} \partial_{\bar{v}} G(z, v) \partial_{\bar{v}} G(v, w) \overline{\mu(v)} d^{2} v \\
& -\frac{1}{2} \rho(z) \overline{\mu(z)} G(z, w) .
\end{aligned}
$$

(v) For $z \neq w$,

$$
\begin{aligned}
& L_{\mu} \partial_{z} \partial_{w} G(z, w)=2 \iint_{F} \partial_{z} \partial_{v} G(z, v) \partial_{w} \partial_{v} G(v, w) \mu(v) d^{2} v \\
& L_{\bar{\mu}} \partial_{z} \partial_{w} G(z, w)=2 \iint_{F} \partial_{z} \partial_{\bar{v}} G(z, v) \partial_{w} \partial_{\bar{v}} G(v, w) \overline{\mu(v)} d^{2} v
\end{aligned}
$$


Both integrals in (ii) and the first integrals in (iii)-(v) are understood in the principal value sense.

Proof. From the definition of the propagator $G(z, w)$ we obtain

$$
\left(L_{\mu}\left(\Delta_{0}+\frac{1}{2}\right)\right) G(z, w)+\left(\Delta_{0}+\frac{1}{2}\right)\left(L_{\mu} G(z, w)\right)=0,
$$

so that using (4.16) we get for $z \neq w$,

$$
\begin{aligned}
L_{\mu} G(z, w) & =-\left(\left(\Delta_{0}+\frac{1}{2}\right)^{-1} \rho^{-1} \partial \mu \partial\right) G(z, w) \\
& =-2 \iint_{F} G(z, v) \partial_{v} \mu(v) \partial_{v} G(v, w) d^{2} v \\
& =2 \iint_{F} \partial_{v} G(z, v) \partial_{v} G(v, w) \mu(v) d^{2} v,
\end{aligned}
$$

which proves (i). Here in the last line we used the Stokes' theorem and elementary fact

$$
\oint_{|z|=\varepsilon} \frac{d \bar{z}}{z}=0
$$

To prove (ii), it is convenient to use the Fuchsian global coordinate on $X \simeq \Gamma \backslash \mathbb{U}$. Using (4.9), we get

$$
\begin{gathered}
L_{\mu} G(z, z)=\lim _{w \rightarrow z} L_{\mu}\left(G(z, w)+\frac{1}{2 \pi}\left(\log |z-w|^{2}+\phi_{c l}(z)\right)\right) \\
=\lim _{w \rightarrow z}\left(2 \iint_{F} \partial_{v} G(z, v) \partial_{v} G(v, w) \mu(v) d^{2} v+\frac{1}{2 \pi}\left(\frac{\dot{f}(z)-\dot{f}(w)}{z-w}-\dot{f}_{z}(z)\right)\right),
\end{gathered}
$$

where it is easy to justify the interchange of the Lie derivative $L_{\mu}$ and the limit $w \rightarrow z$. Using (5.6) and (5.26) we have

$$
\begin{gathered}
2 \iint_{F} \partial_{v} G(z, v) \partial_{v} G(v, w) \mu(v) d^{2} v=2 \iint_{\mathbb{U}} \partial_{v} \mathcal{G}(z, v) \partial_{v} G(v, w) \mu(v) d^{2} v \\
=2 \iint_{\mathbb{U}}\left(-\frac{1}{2 \pi} \frac{1}{v-z}+h_{1}(v, z)\right)\left(-\frac{1}{2 \pi} \frac{1}{v-w}+h_{2}(v, w)\right) \mu(v) d^{2} v \\
=\frac{1}{2 \pi^{2}} \iint_{\mathbb{U}}\left(\frac{1}{(v-z)(v-w)}-\frac{2 \pi}{v-z} h_{2}(v, w)-\frac{2 \pi}{v-w} h_{1}(v, z)\right. \\
\left.+4 \pi^{2} h_{1}(v, z) h_{2}(v, w)\right) \mu(v) d^{2} v,
\end{gathered}
$$

where $h_{1}, h_{2}$ are bounded functions on $\mathbb{U} \times \mathbb{U}$. The last three terms are continuous as $w \rightarrow z$. On the other hand, it follows from (4.3) that

$$
\frac{\dot{f}(z)-\dot{f}(w)}{z-w}-\dot{f}_{z}(z)=-\frac{1}{\pi} \iint_{\mathbb{U}} \mu(v)\left(\frac{1}{(v-z)(v-w)}-\frac{1}{(v-z)^{2}}\right) d^{2} v
$$


where the integral is understood in the principal value sense. Combining this with the previous formula gives (ii).

To prove (iii), we use (4.17)-(4.18) to get for $z \neq w$,

$$
L_{\mu} \partial_{z} G(z, w)=\partial_{z} L_{\mu} G(z, w)=2 \iint_{F} \partial_{z} \partial_{v} G(z, v) \partial_{v} G(v, w) \mu(v) d^{2} v
$$

and

$$
\begin{aligned}
L_{\bar{\mu}} \partial_{z} G(z, w) & =-\overline{\mu(z)} \partial_{\bar{z}} G(z, w)+2 \partial_{z} \iint_{F} \partial_{\bar{v}} G(z, v) \partial_{\bar{v}} G(v, w) \overline{\mu(v)} d^{2} v \\
& =2 \iint_{F} \partial_{z} \partial_{\bar{v}} G(z, v) \partial_{\bar{v}} G(v, w) \overline{\mu(v)} d^{2} v
\end{aligned}
$$

Here we used (5.12) and the elementary formula

$$
\frac{\partial}{\partial z} \iint_{|v-z| \leq \varepsilon} \frac{f(z, v)}{\bar{v}-\bar{z}} d^{2} v=\iint_{|v-z| \leq \varepsilon} \frac{f_{z}(z, v)}{\bar{v}-\bar{z}} d^{2} v-\pi f(z, z)+O(\varepsilon)
$$

where $f(z, v)$ is smooth at $z=v$, which readily follows from the Stokes' theorem.

Parts (iv) and (v) are proved similarly. In particular, using (4.18) we obtain for $z \neq w$,

$$
\begin{aligned}
L_{\bar{\mu}} \mathcal{D}_{z} G(z, w)= & -\overline{\mu(z)} \partial_{z} \partial_{\bar{z}} G(z, w)-\rho(z) \partial_{z} \rho(z)^{-1} \overline{\mu(z)} \partial_{\bar{z}} G(z, w) \\
& +2 \rho(z) \partial_{z} \rho(z)^{-1} \partial_{z} \iint_{F} \partial_{\bar{u}} G(z, u) \partial_{\bar{u}} G(u, w) \overline{\mu(u)} d^{2} u \\
= & -\overline{\mu(z)} \partial_{z} \partial_{\bar{z}} G(z, w)-\rho(z) \partial_{z} \rho(z)^{-1} \overline{\mu(z)} \partial_{\bar{z}} G(z, w) \\
& +2 \rho(z) \partial_{z} \rho(z)^{-1} \iint_{F} \partial_{\bar{u}} \partial_{z} G(z, u) \partial_{\bar{u}} G(u, w) \overline{\mu(u)} d^{2} u \\
& +\rho(z) \partial_{z} \rho(z)^{-1} \overline{\mu(z)} \partial_{\bar{z}} G(z, w) \\
= & -\frac{1}{2} \rho(z) \overline{\mu(z)} G(z, w)+2 \iint_{F} \partial_{\bar{u}} \mathcal{D}_{z} G(z, u) \partial_{\bar{u}} G(u, w) \overline{\mu(u)} d^{2} u
\end{aligned}
$$

Corollary 5.5. Let $h^{\varepsilon}$ be a smooth family of $\Gamma^{\varepsilon \mu}$-automorphic functions on $\Omega^{\varepsilon \mu}$. Then

(i)

$$
L_{\mu} \iint_{F} \mathcal{D}_{z} G(z, u) h(u) \rho(u) d^{2} u=\iint_{F}\left(L_{\mu} \mathcal{D}_{z} G(z, u) h(u)+\mathcal{D}_{z} G(z, u) L_{\mu} h(u)\right) \rho(u) d^{2} u
$$


(ii)

$$
\begin{aligned}
L_{\bar{\mu}} \iint_{F} \mathcal{D}_{z} G(z, u) h(u) \rho(u) d^{2} u= & \iint_{F}\left(L_{\bar{\mu}} \mathcal{D}_{z} G(z, u) h(u)+\mathcal{D}_{z} G(z, u) L_{\bar{\mu}} h(u)\right) \rho(u) d^{2} u \\
& +\frac{1}{2} \rho(z) \overline{\mu(z)} h(z),
\end{aligned}
$$

where integrals are understood in the principal value sense.

Proof. Follows from part (iv) of Lemma (5.4) and definition of the principal value integral.

For a global coordinate $z$ on $X \simeq \Gamma \backslash \Omega$, set

$$
K_{\Gamma}(z, w)=\sum_{\gamma \in \Gamma} \frac{\gamma^{\prime}(w)^{2}}{(z-\gamma w)^{4}} .
$$

The kernel $K_{\Gamma}$ is a meromorphic quadratic differential for $\Gamma$ in $z$ and $w$, with the fourth order pole at $z=w$. When $z$ is the Fuchsian global coordinate, it follows from (5.24) that

$$
K_{\Gamma}(z, w)=\frac{\pi}{3} \mathcal{D}_{z} \mathcal{D}_{w} G(z, w)
$$

Lemma 5.6. Let $z$ be a global coordinate $z$ on $X \simeq \Gamma \backslash \Omega$ and $\mu \in \Omega^{-1,1}(\Gamma)$. For $H(z)=\partial_{z} \partial_{z} G(z, z)$ we have

$$
\begin{aligned}
& L_{\mu} H(z)=2 \iint_{F}\left(\left(\partial_{z} \partial_{w} G(z, w)\right)^{2}-\frac{1}{4 \pi^{2}} K_{\Gamma}(z, w)\right) \mu(w) d^{2} w \\
& L_{\bar{\mu}} H(z)=2 \iint_{F}\left(\partial_{z} \partial_{\bar{w}} G(z, w)\right)^{2} \overline{\mu(w)} d^{2} w-\frac{1}{4 \pi} \rho(z) \overline{\mu(z)} .
\end{aligned}
$$

Proof. Let $z$ be the Fuchsian global coordinate on $X \simeq \Gamma \backslash \mathbb{U}$ and $\left.\mu\right|_{\mathbb{L}}=0$, so that corresponding $\Gamma^{\varepsilon \mu}=f^{\varepsilon \mu} \circ \Gamma \circ\left(f^{\varepsilon \mu}\right)^{-1}$ are quasi-Fuchsian groups for $\varepsilon \neq 0$. In order to use representation (5.16), we need to change a quasiFuchsian global coordinate on $X^{\varepsilon \mu} \simeq \Gamma^{\varepsilon \mu} \backslash \Omega_{1}^{\varepsilon \mu}$, where $\Omega_{1}^{\varepsilon \mu}=f^{\varepsilon \mu}(\mathbb{U})$, to the Fuchsian global coordinate, given the covering $J_{\varepsilon \mu}: \mathbb{U} \rightarrow \Omega_{1}^{\varepsilon \mu}$. It follows from (5.20) that

$$
L_{\mu} H(z)=\lim _{z^{\prime} \rightarrow z} L_{\mu}\left(\partial_{z} \partial_{z^{\prime}} G\left(z, z^{\prime}\right)-\partial_{z} \partial_{z^{\prime}} \mathcal{G}\left(z, z^{\prime}\right)-\frac{1}{12 \pi} S\left(J^{-1}\right)(z)\right) .
$$

Using Remark 4.1, the formula

$$
L_{\mu} \mathcal{G}\left(z, z^{\prime}\right)=2 \iint_{\mathbb{U}} \partial_{w} \mathcal{G}(z, w) \partial_{w} \mathcal{G}\left(w, z^{\prime}\right) \mu(w) d^{2} w
$$


and (5.6) and (5.26), we obtain

$$
\begin{aligned}
L_{\mu} H(z) & =2 \lim _{z^{\prime} \rightarrow z} \iint_{\mathbb{U}} \partial_{z} \partial_{w} \mathcal{G}(z, w) \partial_{z^{\prime}} \partial_{w}\left(G\left(w, z^{\prime}\right)-\mathcal{G}\left(w, z^{\prime}\right)\right) \mu(w) d^{2} w \\
& =2 \sum_{\substack{\gamma \in \Gamma \\
\gamma \neq \text { id }}} \iint_{\mathbb{U}} \partial_{z} \partial_{w} \mathcal{G}(z, w) \partial_{z} \partial_{w} \mathcal{G}(\gamma w, z) \mu(w) d^{2} w \\
& =2 \iint_{F} \sum_{\substack{\gamma_{1}, \gamma_{2} \in \Gamma \\
\gamma_{1} \neq \gamma_{2}}} \partial_{z} \partial_{w} \mathcal{G}\left(z, \gamma_{1} w\right) \partial_{z} \partial_{w} \mathcal{G}\left(\gamma_{2} w, z\right) \mu(w) d^{2} w
\end{aligned}
$$

where integrals are understood in the principal value sense. Below we will show that for all $z \in \mathbb{U}$,

$$
\lim _{\varepsilon \rightarrow 0} \iint_{\mathbb{U}_{\varepsilon}(z)}\left(\partial_{z} \partial_{w} \mathcal{G}(z, w)\right)^{2} \mu(w) d^{2} w=0,
$$

where $\mathbb{U}_{\varepsilon}(z)=\{w \in \mathbb{U}:|w-z| \geq \varepsilon\}$. Thus we get

$$
L_{\mu} H(z)=2 \iint_{F}\left(\partial_{z} \partial_{w} G(z, w)\right)^{2} \mu(w) d^{2} w,
$$

where the integral is understood in the principal value sense. For a global coordinate given by $X=\Gamma \backslash \Omega$ we obtain, using (5.20) and (4.11),

$$
\begin{aligned}
L_{\mu} H(z) & =2 \iint_{F}\left(\partial_{z} \partial_{w} G(z, w)\right)^{2} \mu(w) d^{2} w-\frac{1}{2 \pi^{2}} \iint_{\mathbb{C}} \frac{\mu(w)}{(z-w)^{4}} d^{2} w \\
& =2 \iint_{F}\left(\left(\partial_{z} \partial_{w} G(z, w)\right)^{2}-\frac{1}{4 \pi^{2}} K_{\Gamma}(z, w)\right) \mu(w) d^{2} w
\end{aligned}
$$

To prove (5.38), it is convenient to use the unit disc $\mathbb{D}=\{z \in \mathbb{C}:|z|<1\}$. The kernel $\mathcal{G}(z, w)$ is given by the same formula (5.3) , where now

$$
u(z, w)=\frac{|z-w|^{2}}{\left(1-|z|^{2}\right)\left(1-|w|^{2}\right)} .
$$

Using the Ahlfors' formula

$$
\mu(w)=\frac{3\left(1-|w|^{2}\right)^{2}}{\pi} \iint_{\mathbb{D}} \frac{\mu(\zeta)}{(1-\zeta \bar{w})^{4}} d^{2} \zeta,
$$


we obtain

$$
\begin{gathered}
\lim _{\varepsilon \rightarrow 0} \iint_{\mathbb{U}_{\varepsilon}(z)}\left(\partial_{z} \partial_{w} \mathcal{G}(z, w)\right)^{2} \mu(w) d^{2} w=\lim _{\varepsilon \rightarrow 0} \iint_{\mathbb{D}_{\varepsilon}(z)}\left(\partial_{z} \partial_{w} \mathcal{G}(z, w)\right)^{2} \mu(w) d^{2} w \\
=\lim _{\varepsilon \rightarrow 0} \frac{3}{\pi} \iint_{\mathbb{D}_{\varepsilon}(z)} \iint_{\mathbb{D}}\left(\partial_{z} \partial_{w} \mathcal{G}(z, w)\right)^{2} \frac{\left(1-|w|^{2}\right)^{2}}{(1-\zeta \bar{w})^{4}} \mu(\zeta) d^{2} \zeta d^{2} w \\
=\frac{3}{\pi} \iint_{\mathbb{D}} A(z, \zeta) \mu(\zeta) d^{2} \zeta,
\end{gathered}
$$

where $\mathbb{D}_{\varepsilon}(z)=\{w \in \mathbb{D}:|w-z| \geq \varepsilon\}$ and

$$
A(z, \zeta)=\lim _{\varepsilon \rightarrow 0} \iint_{\mathbb{D}_{\varepsilon}(z)}\left(\partial_{z} \partial_{w} \mathcal{G}(z, w)\right)^{2} \frac{\left(1-|w|^{2}\right)^{2}}{(1-\zeta \bar{w})^{4}} d^{2} w .
$$

Using (5.5), we get $A(\sigma z, \sigma \zeta) \sigma^{\prime}(z)^{2} \sigma^{\prime}(\zeta)^{2}=A(z, \zeta)$ for all $\sigma \in \operatorname{PSU}(1,1)$, and by explicit computation,

$$
\partial_{z} \partial_{w} \mathcal{G}(0, w)=-\frac{1}{2 \pi} \frac{\bar{w}^{2}}{\left(1-|w|^{2}\right)}\left(\frac{1-3|w|^{2}}{|w|^{4}}-\frac{2}{1-|w|^{2}} \log |w|^{2}\right) .
$$

Using polar coordinates, we immediately obtain that $A(0, \zeta)=0$ and, therefore, $A(z, \zeta)=0$ for all $z, \zeta \in \mathbb{D}$.

Finally, using (3.12) and (4.3), we have for a global coordinate $z$ on $X \simeq$ $\Gamma \backslash \Omega$,

$$
\begin{aligned}
L_{\bar{\mu}} H(z) & =\lim _{z^{\prime} \rightarrow z} L_{\bar{\mu}}\left(\partial_{z} \partial_{z^{\prime}} G\left(z, z^{\prime}\right)+\frac{1}{2 \pi}\left(\frac{1}{\left(z-z^{\prime}\right)^{2}}-\frac{1}{2} \rho(z) \frac{\bar{z}-\bar{z}^{\prime}}{z-z^{\prime}}\right)\right) \\
& =\lim _{z^{\prime} \rightarrow z}\left(2 \iint_{F} \partial_{z} \partial_{\bar{w}} G(z, w) \partial_{z^{\prime}} \partial_{\bar{w}} G\left(w, z^{\prime}\right) \overline{\mu(w)} d^{2} w\right. \\
& -\frac{1}{4 \pi} \frac{\rho(z)}{z-z^{\prime}}\left(\overline{\dot{f}(z)}-\overline{\dot{f}\left(z^{\prime}\right)}-\left(\bar{z}-\bar{z}^{\prime}\right) \overline{\left.\dot{f}_{z}(z)\right)}\right) \\
& =2 \iint_{F}\left(\partial_{z} \partial_{\bar{w}} G(z, w)\right)^{2} \overline{\mu(w)} d^{2} w-\frac{1}{4 \pi} \rho(z) \overline{\mu(z)} .
\end{aligned}
$$

On the other hand, using (4.12), we have for the Fuchsian global coordinate on $X \simeq \Gamma \backslash \mathbb{U}$,

$$
\begin{aligned}
L_{\bar{\mu}} H(z) & =2 \iint_{F}\left(\partial_{z} \partial_{\bar{w}} G(z, w)\right)^{2} \overline{\mu(w)} d^{2} w-2 \iint_{\mathbb{U}}\left(\partial_{z} \partial_{\bar{w}} \mathcal{G}(z, w)\right)^{2} \overline{\mu(w)} d^{2} w \\
& +\frac{1}{24 \pi} \rho(z) \overline{\mu(z)} .
\end{aligned}
$$


Hence,

$$
\iint_{\mathbb{U}}\left(\partial_{z} \partial_{\bar{w}} \mathcal{G}(z, w)\right)^{2} \overline{\mu(w)} d^{2} w=\frac{7}{48 \pi} \rho(z) \overline{\mu(z)},
$$

which can be also verified directly.

\section{ONE-POINT CORRELATION FUNCTIONS}

Here we compute one-point correlation functions $\langle\langle T(z) X\rangle\rangle$ and $\langle\langle\bar{T}(\bar{z}) X\rangle\rangle$ in all orders of the perturbation theory.

Theorem 6.1. Let $\mathcal{F}$ be a formal function on the Schottky space $\mathfrak{S}_{g}$, associated with the free energy $\mathcal{F}_{X}$ and defined in Section 4.1. For every $t \in \mathfrak{S}_{g}$ let $X_{t} \simeq \Gamma_{t} \backslash \Omega_{t}$, where $\Gamma_{t}$ is the corresponding Schottky group, and let $J_{t}=J_{S}^{-1} \circ J_{F}$, where $J_{S}$ and $J_{F}$ are covering maps corresponding to the Schottky and Fuchsian uniformizations of $X_{t}$. Then for every $t \in \mathfrak{S}_{g}$ correlation functions $\left\langle\left\langle T(z) X_{t}\right\rangle\right\rangle$ and $\left\langle\left\langle\bar{T}(\bar{z}) X_{t}\right\rangle\right\rangle$ are holomorphic and antiholomorphic quadratic differentials for $\Gamma_{t}$, and

$$
\begin{aligned}
& (\partial \mathcal{F})(t)=\frac{1}{\pi}\left(\left\langle\left\langle T(z) X_{t}\right\rangle\right\rangle-\frac{1}{12} \mathcal{S}\left(J_{t}^{-1}\right)(z)\right), \\
& (\bar{\partial} \mathcal{F})(t)=\frac{1}{\pi}\left(\left\langle\left\langle\bar{T}(\bar{z}) X_{t}\right\rangle\right\rangle-\frac{1}{12} \overline{\mathcal{S}\left(J_{t}^{-1}\right)}(\bar{z})\right),
\end{aligned}
$$

which are understood as equalities in $\frac{1}{\hbar} \Omega^{2,0}\left(\Gamma_{t}\right)[[\hbar]]$ and $\frac{1}{\hbar} \Omega^{0,2}\left(\Gamma_{t}\right)[[\hbar]]$ respectively. The same statement holds for the Teichmüller space $\mathfrak{T}_{g}$.

Remark 6.2. Slightly abusing notations, we will write (6.1)-(6.2) as

$$
\begin{aligned}
& \partial \log \langle X\rangle=-\frac{1}{\pi}\left(\langle\langle T(z) X\rangle\rangle-\frac{1}{12} \mathcal{S}\left(J^{-1}\right)(z)\right), \\
& \bar{\partial} \log \langle X\rangle=-\frac{1}{\pi}\left(\langle\langle\bar{T}(\bar{z}) X\rangle\rangle-\frac{1}{12} \overline{\mathcal{S}\left(J^{-1}\right)}(\bar{z})\right) .
\end{aligned}
$$

These equations are conformal Ward identities with single insertion of the stress-energy tensor for quantum Liouville theory on compact Riemann surfaces. In particular, it follows from (6.3) that $\langle\langle T(z) X\rangle\rangle$ is a formal holomorphic quadratic differential on $\Omega_{t}$, i.e., every term in its perturbative expansion is a holomorphic quadratic differential for $\Gamma_{t}$.

Proof. Since $\mathcal{F}$ is real-valued, equation (6.2) follows from (6.1). We prove (6.1) in all orders of the perturbation theory by verifying it at the classical, one-loop, and higher loops levels. For $t \in \mathfrak{S}_{g}$ we will abbreviate $\Gamma=\Gamma_{t}$, $X=X_{t}, J=J_{t}$, etc.

6.1. Classical contribution. Formula (4.10) gives (6.1) at the classical level. 
6.2. One-loop contribution. According to (3.9)-(3.12),

$$
\langle\langle T(z) X\rangle\rangle_{1-\text { loop }}=-\pi\left(H(z)+\iint_{F} \mathcal{D}_{z} G(z, w) G(w, w) \rho(w) d^{2} w\right),
$$

where $H(z)=\partial_{z} \partial_{z} G(z, z)$ and $F$ is a fundamental domain for $\Gamma$ in $\Omega$, and is given by the following graphs (see Fig. 2):

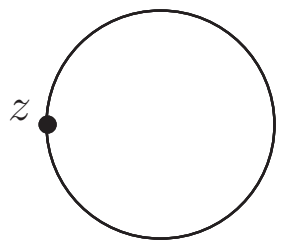

Graph 1

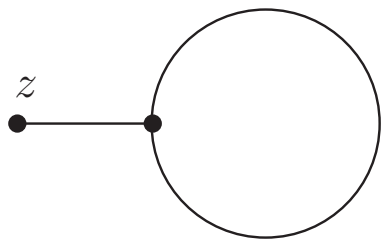

Graph 2

Figure 2

On the other hand, it follows from Definition 3.1 that at the one-loop level $\mathcal{F}_{X}=\frac{1}{2} \log Z(2)$. It was proved in [TZ91, Section 3], using the Fuchsian global coordinate on $X \simeq \Gamma \backslash \mathbb{U}$ that

$$
\partial \log Z(2)=-2 P(H),
$$

where $P$ is an orthogonal projection operator onto the space of holomorphic quadratic differentials.

Remark 6.3. For any choice of a global coordinate on $X$,

$$
\partial \log Z(2)=-2 P\left(H+\frac{1}{12 \pi} \mathcal{S}\left(J^{-1}\right)\right) .
$$

Using (6.5), representation (5.27) and the Stokes' theorem, we get

$$
\begin{aligned}
\partial \log Z(2) & =-8 \iint_{F} \mathcal{D}_{z} \mathcal{D}_{\bar{w}} G(z, w) H(w) \rho(w)^{-1} d^{2} w \\
& =-2 \lim _{\varepsilon \rightarrow 0} \iint_{F_{\varepsilon}(z)} \partial_{\bar{w}} R(w, z) H(w) d^{2} w \\
& =2 \iint_{F} R(w, z) \partial_{\bar{w}} H(w) d^{2} w+i \int_{\partial F_{\varepsilon}(z)} R(w, z) H(w) d w .
\end{aligned}
$$

Here $R(z, w)$ is given by (5.29) and $F_{\varepsilon}(z)=F \backslash\{|w-z| \leq \varepsilon\}$. Since $R(w, z) H(w)$ is a $(1,0)$ tensor for $\Gamma$, the line integral over $\partial F$ vanishes. Using 
(5.30), we get for the remaining line integral, where $C_{\varepsilon}(z)=\{|w-z|=\varepsilon\} 8$,

$$
-i \lim _{\varepsilon \rightarrow 0} \oint_{C_{\varepsilon}(z)} R(w, z) H(w) d w=-2 H(z) .
$$

Now using (5.18), (5.31), (5.35) and the Stokes' theorem, we obtain

$$
\begin{gathered}
2 \iint_{F} R(w, z) \partial_{\bar{w}} H(w) d^{2} w=\iint_{F} R(w, z) \partial_{w}(G(w, w)) \rho(w) d^{2} w \\
=\lim _{\varepsilon \rightarrow 0}\left(-\iint_{F_{\varepsilon}(z)} \partial_{w}(\rho(w) R(w, z)) G(w, w) d^{2} w-\frac{i}{2} \int_{C_{\varepsilon}(z)} R(w, z) G(w, w) d \bar{w}\right) \\
=-2 \iint_{F} \mathcal{D}_{z} G(z, w) G(w, w) \rho(w) d^{2} w .
\end{gathered}
$$

Thus in terms of the the Fuchsian global coordinate on $X$ we have

$$
\partial \log \langle X\rangle_{1-\text { loop }}=-\frac{1}{\pi}\langle\langle T(z) X\rangle\rangle_{1-\text { loop }}
$$

Now using (5.20) and Remark 6.3, we get for the Schottky global coordinate on $X$,

$$
\partial \log \langle X\rangle_{1-\text { loop }}=-\frac{1}{\pi}\left(\langle\langle T(z) X\rangle\rangle_{1-\text { loop }}-\frac{1}{12} \mathcal{S}\left(J^{-1}\right)(z)\right),
$$

where $J=J_{S}^{-1} \circ J_{F}$.

6.3. Higher loops. The higher loop terms in $\mathcal{F}_{X}$ do not depend on the choice of a global coordinate on $X$, and for convenience we will be using the Fuchsian global coordinate.

Define the "forgetful map" $p_{1}: \mathcal{G}_{\{z\}}^{(c)} \rightarrow \mathcal{G}_{\geq 3}^{(c)}$ by eliminating the labeled vertex of valency 1 or 2 . Namely, if $\Upsilon$ is a graph with a labeled vertex $v_{1}$ of valency 1 and $e$ is the edge connecting it to a vertex $v_{2}$ of valency larger than 3 , then $p_{1}(\Upsilon)$ is obtained by removing the vertex $v_{1}$ and the edge $e$. If the valency of $v_{2}$ is 3 , we also remove the vertex $v_{2}$ and replace two remaining edges at $v_{2}$ by a single edge. If $\Upsilon$ is a graph with a labeled vertex $v_{1}$ of valency 2 with edges $e_{1}$ and $e_{2}$, then $p_{1}(\Upsilon)$ is obtained by removing the vertex $v_{1}$ and replacing the edges $e_{1}, e_{2}$ by a single edge. Clearly, $\chi(\Upsilon)=\chi\left(p_{1}(\Upsilon)\right)$ and $\operatorname{Aut}(\Upsilon)=\operatorname{Aut}\left(p_{1}(\Upsilon)\right)$. Conversely, if $\Upsilon^{\prime} \in p_{1}^{-1}(\Upsilon)$, then $\Upsilon^{\prime}$ is obtained from $\Upsilon$ by one of the following ways.

(a) Attach an extra edge $e$ to the midpoint of an edge of $\Upsilon$, so that one of its endpoints becomes a vertex of valency 3 , and the other becomes a labeled vertex of valency 1 .

(b) Insert a labeled vertex $v$ of valency 2 at the midpoint of an edge of $\Upsilon$.

\footnotetext{
${ }^{8}$ Here and in what follows all contours like $C_{\varepsilon}(z)$ are oriented counter-clockwise.
} 
(c) Attach an extra edge $e$ to a vertex $v$ of valency $n$ of $\Upsilon$, so that $v$ becomes a vertex of valency $n+1$, and the other endpoint of $e$ becomes a labeled vertex of valency 1 .

We have that in case (a) $V^{\prime}=V+2$ and $\varepsilon_{1}\left(\Upsilon^{\prime}\right)=1$, in case (b) $V^{\prime}=V+1$ and $\varepsilon_{1}\left(\Upsilon^{\prime}\right)=0$, and in case (c) $V^{\prime}=V+1$ and $\varepsilon_{1}\left(\Upsilon^{\prime}\right)=1$.

We will show that at the higher loop level equation (6.1) is valid graph by graph, i.e., for every $\Upsilon \in \mathcal{G}_{\geq 3}^{(c)}$ with more than one loop,

$$
\partial W_{\Upsilon}(X)=-2 \sum_{\Upsilon^{\prime} \in p_{1}^{-1}(\Upsilon)}(-1)^{|V(\Upsilon)|+\left|V\left(\Upsilon^{\prime}\right)\right|+\varepsilon_{1}\left(\Upsilon^{\prime}\right)} W_{\Upsilon^{\prime}}(X ; z) .
$$

Using (3.6)-(3.7) and the Leibniz rule, we get

$$
\begin{gathered}
L_{\mu} W_{\Upsilon}(X)=\sum_{e \in E(\Upsilon)} \iint_{X} \iint_{X} L_{\mu} G\left(P_{v_{0}(e)}, P_{v_{1}(e)}\right) W_{\Upsilon}^{e}(X)\left(P_{v_{0}(e)}, P_{v_{1}(e)}\right) \\
d P_{v_{0}(e)} d P_{v_{1}(e)},
\end{gathered}
$$

where

$$
W_{\Upsilon}^{e}(X)\left(P_{v_{0}(e)}, P_{v_{1}(e)}\right)=\int \ldots \int \prod_{X^{V^{\prime}}} \prod_{e^{\prime} \in E(\Upsilon) \backslash\{e\}} G\left(P_{v_{0}\left(e^{\prime}\right)}, P_{v_{1}\left(e^{\prime}\right)}\right) \prod_{k=1}^{V^{\prime}} d P_{k} .
$$

Here $V^{\prime}=V-2$ unless $v_{0}(e)=v_{1}(e)$, in which case $V^{\prime}=V-1$ and there is a single integration over $P_{v_{0}(e)}$ in (6.9).

First we consider the case $v_{0}(e) \neq v_{1}(e)$. Using part (i) of Lemma 5.4, we get that the contribution of an edge $e$ into $L_{\mu} W_{\Upsilon}(X)$ is

$2 \iint_{F} \iint_{F} W_{\Upsilon}^{e}\left(z_{1}, z_{2}\right)\left(\iint_{F} \partial_{z} G\left(z_{1}, z\right) \partial_{z} G\left(z_{2}, z\right) \mu(z) d^{2} z\right) \rho\left(z_{1}\right) \rho\left(z_{2}\right) d^{2} z_{1} d^{2} z_{2}$.

Using (5.27), we get that the contribution of the edge $e$ to $\partial W_{\Upsilon}(X)$ is

$$
\iint_{F} \iint_{F} W_{\Upsilon}^{e}\left(z_{1}, z_{2}\right) I\left(z_{1}, z_{2}\right) \rho\left(z_{1}\right) \rho\left(z_{2}\right) d^{2} z_{1} d^{2} z_{2}
$$

where

$$
I\left(z_{1}, z_{2}\right)=8 \iint_{F} \mathcal{D}_{z} \mathcal{D}_{\bar{w}} G(z, w) \partial_{w} G\left(z_{1}, w\right) \partial_{w} G\left(z_{2}, w\right) \rho(w)^{-1} d^{2} w
$$

and the change of the order of integrations is easily justified. Using the Stokes' theorem and setting $F_{\varepsilon}\left(z, z_{1}, z_{2}\right)=F_{\varepsilon}(z) \backslash\left\{\left\{\left|w-z_{1}\right| \leq \varepsilon\right\} \cup\left\{\left|w-z_{2}\right| \leq \varepsilon\right\}\right\}$, 


$$
\begin{aligned}
C_{\varepsilon}\left(z_{1}, z_{2}\right) & =\left\{\left|w-z_{1}\right|=\varepsilon\right\} \cup\left\{\left|w-z_{2}\right|=\varepsilon\right\}, \text { we get } \\
I & =-2 \lim _{\varepsilon \rightarrow 0} \iint_{F_{\varepsilon}\left(z, z_{1}, z_{2}\right)} R(w, z) \partial_{\bar{w}}\left(\partial_{w} G\left(z_{1}, w\right) \partial_{w} G\left(z_{2}, w\right)\right) d^{2} w \\
& +i \lim _{\varepsilon \rightarrow 0} \oint_{C_{\varepsilon}(z)} R(w, z) \partial_{w} G\left(z_{1}, w\right) \partial_{w} G\left(z_{2}, w\right) d w \\
& +i \lim _{\varepsilon \rightarrow 0} \oint_{C_{\varepsilon}\left(z_{1}, z_{2}\right)} R(w, z) \partial_{w} G\left(z_{1}, w\right) \partial_{w} G\left(z_{2}, w\right) d w \\
& =I_{1}+I_{2}+I_{3} .
\end{aligned}
$$

As in the one-loop case, using property P3, (5.12) and (5.31), we obtain for $z \neq z_{1}, z_{2}$,

$$
\begin{aligned}
I_{1} & =-\iint_{F} \rho(w) R(w, z) \partial_{w}\left(G\left(z_{1}, w\right) G\left(z_{2}, w\right)\right) d^{2} w \\
& =2 \iint_{F} \mathcal{D}_{z} G(z, w) G\left(z_{1}, w\right) G\left(z_{2}, w\right) \rho(w) d^{2} w \\
I_{2} & =2 \partial_{z} G\left(z_{1}, z\right) \partial_{z} G\left(z_{2}, z\right) \\
I_{3} & =\left(R\left(z_{1}, z\right) \partial_{z_{1}}+R\left(z_{2}, z\right) \partial_{z_{2}}\right) G\left(z_{1}, z_{2}\right) .
\end{aligned}
$$

Now it follows from (3.10)-(3.11) that the terms $I_{1}$ and $I_{2}$ correspond, respectively, to the contribution into (6.8) of graphs of type (a) and (b) such that the corresponding edge $e$ is not a loop. Assuming that there are no self-loops starting at $v_{0}(e)$ and $v_{1}(e)$, we can collect terms $I_{3}$ corresponding to all edges having $v_{0}(e)$ or $v_{1}(e)$ as their endpoints. This gives

$$
\begin{gathered}
\iint_{F} W_{\Upsilon}^{z_{1}} \rho\left(z_{1}\right) R\left(z_{1}, z\right) \partial_{z_{1}} \prod_{k=1}^{n_{1}} G\left(z_{1}, u_{k}\right) d^{2} z_{1} \\
+\iint_{F} W_{\Upsilon}^{z_{2}} \rho\left(z_{2}\right) R\left(z_{2}, z\right) \partial_{z_{2}} \prod_{l=1}^{n_{2}} G\left(z_{2}, v_{l}\right) d^{2} z_{2} \\
=-2 \iint_{F} W_{\Upsilon}^{z_{1}} \mathcal{D}_{z} G\left(z, z_{1}\right) \prod_{k=1}^{n_{1}} G\left(z_{1}, u_{k}\right) \rho\left(z_{1}\right) d^{2} z_{1} \\
-2 \iint_{F} W_{\Upsilon}^{z_{2}} \mathcal{D}_{z} G\left(z, z_{2}\right) \prod_{l=1}^{n_{2}} G\left(z_{2}, v_{l}\right) \rho\left(z_{2}\right) d^{2} z_{2},
\end{gathered}
$$

where $u_{1}, \ldots, u_{n_{1}}$ and $v_{1}, \ldots, v_{n_{2}}$, respectively, parameterize all vertices in the stars of $v_{1}(e)$ and $v_{2}(e)$. These terms correspond to the contribution into (6.8) from the graphs of type (c) such that there are no self-loops starting at $v_{0}(e)$ and $v_{1}(e)$. 
For the remaining case when $v_{0}(e)=v_{1}(e)$, or when there are self-loops starting at $v_{0}(e)$ or $v_{1}(e)$, we consider the principal value integral

$$
\begin{aligned}
\tilde{I} & =8 \iint_{F} \mathcal{D}_{z} \mathcal{D}_{\bar{w}} G(z, w)\left(\partial_{w} G\left(z_{1}, w\right)\right)^{2} \rho(w)^{-1} d^{2} w \\
& =\tilde{I}_{1}+\tilde{I}_{2}+\tilde{I}_{3}
\end{aligned}
$$

where as in the previous case,

$$
\begin{aligned}
& \tilde{I}_{1}=2 \iint_{F} \mathcal{D}_{z} G(z, w) G\left(z_{1}, w\right)^{2} \rho(w) d^{2} w, \\
& \tilde{I}_{2}=2\left(\partial_{z} G\left(z_{1}, z\right)\right)^{2}, \\
& \tilde{I}_{3}=i \lim _{\varepsilon \rightarrow 0} \oint_{C_{\varepsilon}\left(z_{1}\right)} R(w, z)\left(\partial_{w} G\left(z_{1}, w\right)\right)^{2} d w .
\end{aligned}
$$

It follows from (3.10)-(3.11) that the terms $\tilde{I}_{1}$ and $\tilde{I}_{2}$ correspond, respectively, to the contribution into (6.8) of the graphs of type (a) and (b) such that the corresponding edge $e$ is a loop. To evaluate $\tilde{I}_{3}$, we use

$$
\begin{aligned}
\left(\partial_{w} G\left(z_{1}, w\right)\right)^{2} & =\sum_{\gamma_{1}, \gamma_{2} \in \Gamma} \partial_{w} \mathcal{G}\left(\gamma_{1} z_{1}, w\right) \partial_{w} \mathcal{G}\left(\gamma_{2} z_{1}, w\right) \\
& =\sum_{\gamma_{1} \neq \gamma_{2} \in \Gamma} \partial_{w} \mathcal{G}\left(\gamma_{1} z_{1}, w\right) \partial_{w} \mathcal{G}\left(\gamma_{2} z_{1}, w\right)+\sum_{\gamma \in \Gamma}\left(\partial_{w} \mathcal{G}\left(\gamma z_{1}, w\right)\right)^{2}
\end{aligned}
$$

and write $\tilde{I}_{3}=\tilde{I}_{3,1}+\tilde{I}_{3,2}$. Using (5.9) and (5.5) we obtain

$$
\begin{aligned}
\tilde{I}_{3,1} & =i \sum_{\gamma_{1} \neq \gamma_{2} \in \Gamma} \lim _{\varepsilon \rightarrow 0} \oint_{C_{\varepsilon}\left(z_{1}\right)} R(w, z) \partial_{w} \mathcal{G}\left(\gamma_{1} z_{1}, w\right) \partial_{w} \mathcal{G}\left(\gamma_{2} z_{1}, w\right) d w \\
& =\left.2 \sum_{\gamma \neq \mathrm{id} \in \Gamma} R\left(z_{1}, z\right) \partial_{w} \mathcal{G}\left(\gamma z_{1}, w\right)\right|_{w=z_{1}}=R\left(z_{1}, z\right) \partial_{z_{1}} G\left(z_{1}, z_{1}\right),
\end{aligned}
$$

and using property (5.31) we get

$$
\tilde{I}_{3,2}=i \sum_{\gamma \in \Gamma} \lim _{\varepsilon \rightarrow 0} \oint_{C_{\varepsilon}\left(z_{1}\right)} R(w, z)\left(\partial_{w} \mathcal{G}\left(\gamma z_{1}, w\right)\right)^{2} d w=-\frac{1}{\pi} \mathcal{D}_{z} G\left(z, z_{1}\right) .
$$

Now collecting all terms $\tilde{I}_{3,1}$ corresponding to edges having the vertex $v_{0}(e)=v_{1}(e)$ as an endpoint, we get

$$
\begin{aligned}
& \iint_{F} W_{\Upsilon}^{z_{1}} \rho\left(z_{1}\right) R\left(z_{1}, z\right) \partial_{z_{1}} \prod_{k=1}^{n_{1}} G\left(z_{1}, u_{k}\right) d^{2} z_{1} \\
= & -2 \iint_{F} W_{\Upsilon}^{z_{1}} \mathcal{D}_{z} G\left(z, z_{1}\right) \prod_{k=1}^{n_{1}} G\left(z_{1}, u_{k}\right) \rho\left(z_{1}\right) d^{2} z_{1},
\end{aligned}
$$


and collecting all terms $\tilde{I}_{3,2}$ we obtain

$$
-\frac{m_{1}}{\pi} \iint_{F} W_{\Upsilon}^{z_{1}} \mathcal{D}_{z} G\left(z, z_{1}\right) \prod_{k=2}^{n_{1}} G\left(z_{1}, u_{k}\right) \rho\left(z_{1}\right) d^{2} z_{1},
$$

where $m_{1}$ is the number of self-loops at the vertex $v_{0}(e)=v_{1}(e)$. Thus in accordance with the Feynman rules in Section 3.2 , terms $\tilde{I}_{3}$ correspond to the contribution into (6.8) of the remaining graphs of type (c).

Remark 6.4. It is elementary to show, using (5.6) and (5.21), that

$$
\iint_{F} \mathcal{D}_{z} G(z, w) \rho(w) d^{2} w=0
$$

so that

$$
\iint_{F} \mathcal{D}_{z} G(z, w) G(w, w) \rho(w) d^{2} w=\iint_{F} \mathcal{D}_{z} G(z, w)\left(G(w, w)+\frac{1}{2 \pi}\right) \rho(w) d^{2} w .
$$

Thus the Feynman rule for vertices with self-loops is consistent with the regularization at the one loop level.

\section{TwO-POINT CORRELATION FUNCTION $-T T$ EQUATION}

Here we compute two point correlation functions $\langle\langle T(z) T(w) X\rangle\rangle$ and $\langle\langle\bar{T}(\bar{z}) \bar{T}(\bar{w}) X\rangle\rangle$ in all orders of perturbation theory. Namely, we express them through one point correlation functions $\langle\langle T(z) X\rangle\rangle$ and $\langle\langle\bar{T}(\bar{z}) X\rangle\rangle$, which according to Theorem 6.1 can be considered as formal $(1,0)$ and $(0,1)$-forms on the Schottky space $\mathfrak{S}_{g}$. Using notations in Section 4.3, we have the following result.

Theorem 7.1. For every $t \in \mathfrak{S}_{g}$ correlation functions $\left\langle\left\langle T(z) T(w) X_{t}\right\rangle\right\rangle$ and $\left\langle\left\langle\bar{T}(\bar{z}) \bar{T}(\bar{w}) X_{t}\right\rangle\right\rangle$, where $X_{t} \simeq \Gamma_{t} \backslash \Omega_{t}$, are meromorphic and anti-meromorphic quadratic differentials for $\Gamma_{t}$ respectively, having only fourth order poles at $z=w$ and $\bar{z}=\bar{w}$. For $t \in U_{0} \subset \mathfrak{S}_{g}-$ a coordinate chart of the origin of the Schottky space $\mathfrak{S}_{g}$ - we have

$$
\begin{aligned}
\partial_{s}\left\langle\left\langle T(z) X_{t}\right\rangle\right\rangle= & -\frac{1}{\pi}\left(\left\langle\left\langle T(z) T(w) X_{t}\right\rangle\right\rangle-\frac{c}{2} K_{\Gamma_{t}}(z, w)\right) \\
& +\left(2 \partial_{z} R(z, w)+R(z, w) \partial_{z}\right)\left\langle\left\langle T(z) X_{t}\right\rangle\right\rangle+\mathscr{T}(z, w), \\
\bar{\partial}_{s}\left\langle\left\langle\bar{T}(\bar{z}) X_{t}\right\rangle\right\rangle= & -\frac{1}{\pi}\left(\left\langle\left\langle\bar{T}(\bar{z}) \bar{T}(\bar{w}) X_{t}\right\rangle\right\rangle-\frac{c}{2} K_{\Gamma_{t}}(\bar{z}, \bar{w})\right) \\
& +\left(2 \partial_{\bar{z}} R(\bar{z}, \bar{w})+R(\bar{z}, \bar{w}) \partial_{\bar{z}}\right)\langle\langle\bar{T}(\bar{z}) X\rangle\rangle+\overline{\mathscr{T}(z, w)} .
\end{aligned}
$$

Here $c=\frac{12}{\hbar}+1$ and $\mathscr{T}(z, w)=\sum_{n=-1}^{\infty} \hbar^{n} T_{n}(z, w)$ with $T_{n}(z, w)$ are smooth quadratic differentials for $\Gamma_{t}$ in $z$ and $w$ which are holomorphic in $w$. Kernels $R(z, w)$ and $K_{\Gamma}(z, w)$ are given explicitly by (5.29) and (5.36) respectively, and $R(\bar{z}, \bar{w})=\overline{R(z, w)}, K_{\Gamma}(\bar{z}, \bar{w})=\overline{K_{\Gamma}(z, w)}$. The same statement holds for the Teichmüller space $\mathfrak{T}_{g}$. 
Equations (7.1)-(7.2) are conformal Ward identities with two insertions of same type components of the stress-energy tensor for quantum Liouville theory on compact Riemann surfaces.

Proof. For $t \in \mathfrak{S}_{g}$ we abbreviate $\Gamma=\Gamma_{t}, X=X_{t}, J=J_{t}, K=K_{\Gamma_{t}}$, etc. Equation (7.2) follows from (7.1), and we will prove (7.1) by computing $\partial_{s}\langle\langle T(z) X\rangle\rangle$ in all orders of perturbation theory.

7.1. Classical contribution. As it follows from (4.11),

$$
L_{\nu} T_{c l}(z)=\frac{6}{\pi \hbar} \iint_{\mathbb{C}} \frac{\nu(w)}{(z-w)^{4}} d^{2} w=\frac{6}{\pi \hbar} \iint_{F} K(z, w) \nu(w) d^{2} w,
$$

where $F$ is a fundamental domain for $\Gamma$ in $\Omega$. Using Stokes' theorem, (5.30) and (5.32), we get

$$
\begin{gathered}
P_{w}(K)(z, w)=4 \iint_{\Gamma \backslash \Omega} \mathcal{D}_{w} \mathcal{D}_{\bar{u}} G(w, u) K(z, u) \rho(u)^{-1} d^{2} u \\
=\frac{i}{2} \lim _{\varepsilon \rightarrow 0}\left(\oint_{C_{\varepsilon}(w)} R(u, w) K(z, u) d u+\oint_{C_{\varepsilon}(z)} R(u, w) K(z, u) d u\right) \\
=K(z, w)-\frac{\pi}{3} \mathcal{D}_{z} \mathcal{D}_{w} G(z, w)+\frac{\pi \hbar}{6}\left(2 \partial_{z} R(z, w)+R(z, w) \partial_{z}\right) T_{c l}(z) .
\end{gathered}
$$

It follows from Remark 5.2 that $P_{w}(K)(z, w)$, in agreement with (5.28), is smooth quadratic differential in $z$ and $w$ which is holomorphic in $w$, and such that $\left(P_{w}(K)(z, \cdot), \nu\right)=(K(z, \cdot), \nu)$ for all $\nu \in \Omega^{-1,1}(\Gamma)$. In particular,

$$
\iint_{F}\left(2 \mathcal{D}_{z} \mathcal{D}_{w} G(z, w)-\hbar\left(2 \partial_{z} R(z, w)+R(z, w) \partial_{z}\right) T_{c l}(z)\right) \nu(w) d^{2} w=0 .
$$

On the other hand,

$$
\langle\langle T(z) T(w) X\rangle\rangle_{c l}=\frac{2 \pi}{\hbar} \mathcal{D}_{z} \mathcal{D}_{w} G(z, w)
$$

is a meromorphic quadratic differential in variables $z$ and $w$ which corresponds to the single tree graph (see Fig. 3):

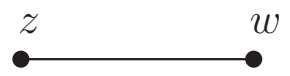

Figure 3

Thus we obtain

$$
\begin{aligned}
\partial_{s} T_{c l}(z)= & \frac{6}{\pi \hbar} P_{z}\left(P_{w}(K)\right)(z, w)=-\frac{1}{\pi}\left(\langle\langle T(z) T(w) X\rangle\rangle_{c l}-\frac{6}{\hbar} K(z, w)\right) \\
& +2 T_{c l}(z) \partial_{z} R(z, w)+\partial_{z} T_{c l}(z) R(z, w)+\frac{6}{\pi \hbar} T(z, w)
\end{aligned}
$$


where $T(z, w)=P_{z}\left(P_{w}(K)\right)(z, w)-P_{w}(K)(z, w)$ is a smooth quadratic differential in variables $z$ and $w$, holomorphic in $w$. Note that since $\partial T_{c l}(z)=0$, the kernel $P_{z}\left(P_{w}(K)\right)(z, w)$ is symmetric.

7.2. One-loop contribution. At the one loop level,

$$
\langle\langle T(z) X\rangle\rangle_{1-\text { loop }}=-\pi\left(H(z)+\iint_{F} \mathcal{D}_{z} G(z, u) G(u, u) \rho(u) d^{2} u\right),
$$

corresponding to two graphs in Fig. 2. On the other hand, it is easy to see that there are eight graphs at the one loop level that have two vertices with

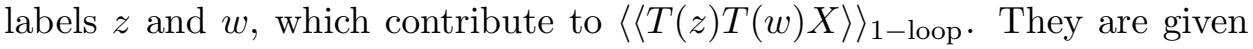
by Fig. 4:

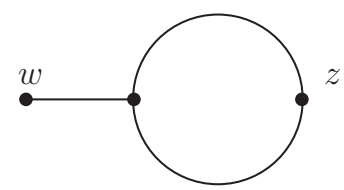

Graph 1

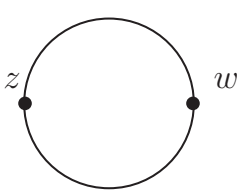

Graph 2
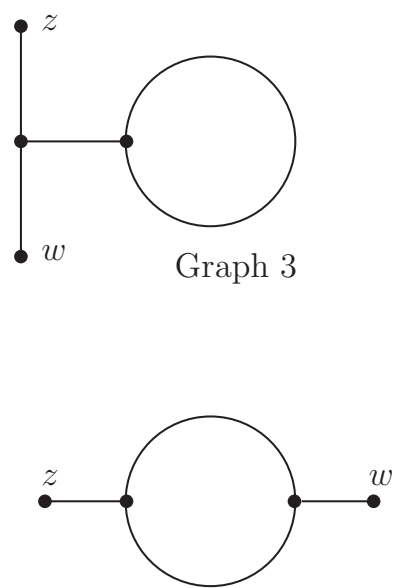

Graph 6

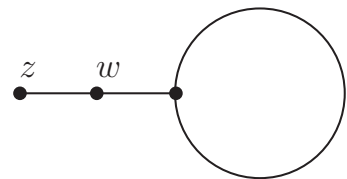

Graph 4

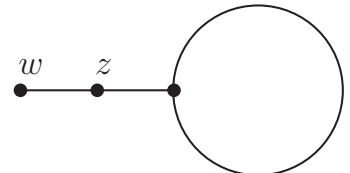

Graph 5

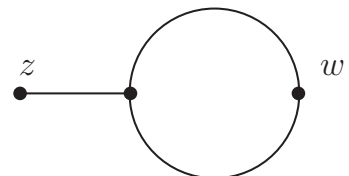

Graph 7

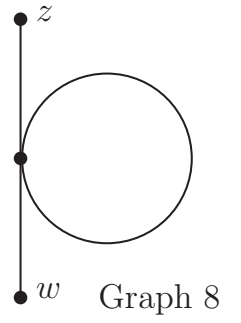

Figure 4 
Using parts (ii) and (iv) of Lemma [5.4, Corollary [5.5] and Lemma 5.6, we get

$$
\begin{aligned}
L_{\nu}\langle\langle T(z) X\rangle\rangle_{1-\text { loop }}= & -\pi\left(L_{\nu} H(z)+\iint_{F} L_{\nu} \mathcal{D}_{z} G(z, u) G(u, u) \rho(u) d^{2} u\right. \\
& \left.+\iint_{F} \mathcal{D}_{z} G(z, u) L_{\nu} G(u, u) \rho(u) d^{2} u\right) \\
= & -\pi \iint_{F} E(z, w) \nu(w) d^{2} w
\end{aligned}
$$

where $E(z, w)=E_{1}(z, w)+E_{2}(z, w)+E_{3}(z, w)$ and

$$
\begin{aligned}
& E_{1}(z, w)=2\left(\partial_{z} \partial_{w} G(z, w)\right)^{2}-\frac{1}{2 \pi^{2}} K(z, w), \\
& E_{2}(z, w)=2 \iint_{F} \mathcal{D}_{z} \partial_{w} G(z, w) \partial_{w} G(w, u) G(u, u) \rho(u) d^{2} u, \\
& E_{3}(z, w)=2 \iint_{F} \mathcal{D}_{z} G(z, u)\left(\partial_{w} G(w, u)\right)^{2} \rho(u) d^{2} u .
\end{aligned}
$$

According to Remark 5.2, $P_{w}\left(E_{i}\right)(z, w), i=1,2,3$, are holomorphic quadratic differentials in $w$. We compute them by using the Fuchsian global coordinate on $X \simeq \Gamma \backslash \mathbb{U}$, so $K(z, w)$ now stands for the kernel (5.36) for the Fuchsian group $\Gamma$. From the Stokes' theorem and property P3 it follows that

$$
\begin{aligned}
P\left(E_{1}\right)= & 4 \iint_{F} \mathcal{D}_{w} \mathcal{D}_{\bar{u}} G(w, u)\left(2\left(\partial_{z} \partial_{u} G(z, u)\right)^{2}-\frac{1}{2 \pi^{2}} K(z, u)\right) \rho(u)^{-1} d^{2} u \\
= & -\iint_{F} \rho(u) R(u, w) \partial_{u}\left(\partial_{z} G(z, u)\right)^{2} d^{2} u \\
& +i \lim _{\varepsilon \rightarrow 0} \oint_{C_{\varepsilon}(w)} R(u, w)\left(\left(\partial_{z} \partial_{u} G(z, u)\right)^{2}-\frac{1}{4 \pi^{2}} K(z, u)\right) d u \\
& +i \lim _{\varepsilon \rightarrow 0} \oint_{C_{\varepsilon}(z)} R(u, w)\left(\left(\partial_{z} \partial_{u} G(z, u)\right)^{2}-\frac{1}{4 \pi^{2}} K(z, u)\right) d u \\
= & T_{1}+T_{2}+T_{3} .
\end{aligned}
$$

Using again the Stokes' theorem, (5.31), and observing that

$$
\lim _{\varepsilon \rightarrow 0} \oint_{C_{\varepsilon}(z)} \rho(u) R(u, w)\left(\partial_{z} G(z, u)\right)^{2} d \bar{u}=0
$$

we obtain

$$
T_{1}=2 \iint_{F} \mathcal{D}_{w} G(u, w)\left(\partial_{z} G(z, u)\right)^{2} \rho(u) d^{2} u
$$


The term $T_{1}$ corresponds to the contribution of graph 1 into $\langle\langle T(z) T(w) X\rangle\rangle_{1-\text { loop }}$. Using (5.30), we get

$$
T_{2}=2\left(\partial_{z} \partial_{w} G(z, w)\right)^{2}-\frac{1}{2 \pi^{2}} K(z, w)
$$

where the first term corresponds to the graph 2 . Since

$$
\left(\partial_{z} \partial_{u} G(z, u)\right)^{2}-\frac{1}{4 \pi^{2}} K(z, u)=2 \partial_{z} \partial_{u} \mathcal{G}(z, u) \sum_{\gamma \neq \mathrm{id} \in \Gamma} \partial_{z} \partial_{u} \mathcal{G}(\gamma z, u) \gamma^{\prime}(z)+O(1)
$$

as $u \rightarrow z$, using (5.17) we obtain

$$
T_{3}=\left(2 \partial_{z} R(z, w)+R(z, w) \partial_{z}\right) H(z) .
$$

To compute $P_{w}\left(E_{2}\right)$ we observe that by the Stokes' theorem and property P3,

$$
\begin{gathered}
8 \iint_{F} \mathcal{D}_{w} \mathcal{D}_{\bar{u}} G(w, u) \mathcal{D}_{z} \partial_{u} G(z, u) \partial_{u} G(u, v) \rho(u)^{-1} d^{2} u \\
=-\iint_{F} \rho(u) R(u, w) \partial_{u}\left(\mathcal{D}_{z} G(z, u) G(u, v)\right) d^{2} u \\
+i \lim _{\varepsilon \rightarrow 0} \oint_{C_{\varepsilon}} R(u, w) \mathcal{D}_{z} \partial_{u} G(z, u) \partial_{u} G(u, v) d u \\
=2 \iint_{F} \mathcal{D}_{w} G(w, u) \mathcal{D}_{z} G(z, u) G(u, v) \rho(u) d^{2} u+2 \mathcal{D}_{z} \partial_{w} G(z, w) \partial_{w} G(w, v) \\
+R(v, w) \mathcal{D}_{z} \partial_{v} G(z, v)+2 \mathcal{D}_{w} \partial_{z} G(z, w) \partial_{z} G(z, v) \\
+\left(2 \partial_{z} R(z, w)+R(z, w) \partial_{z}\right) \mathcal{D}_{z} G(z, v),
\end{gathered}
$$

where $C_{\varepsilon}=C_{\varepsilon}(v) \cup C_{\varepsilon}(w) \cup C_{\varepsilon}(z)$, and in the last line we used (5.30) and (5.33). Thus we obtain

$$
\begin{aligned}
P_{w}\left(E_{2}\right)(z, w) & =8 \iint_{F} \iint_{F} \mathcal{D}_{w} \mathcal{D}_{\bar{u}} G(w, u) \mathcal{D}_{z} \partial_{u} G(z, u) \partial_{u} G(u, v) G(v, v) \rho(u)^{-1} \rho(v) d^{2} u d^{2} v \\
& =T_{4}+T_{5}+T_{6}+T_{7}+T_{8}
\end{aligned}
$$

where

$$
T_{4}=2 \iint_{F} \iint_{F} \mathcal{D}_{w} G(w, u) \mathcal{D}_{z} G(z, u) G(u, v) G(v, v) \rho(u) \rho(v) d^{2} u d^{2} v
$$

and

$$
T_{5}=2 \iint_{F} \mathcal{D}_{z} \partial_{w} G(z, w) \partial_{w} G(w, v) G(v, v) \rho(v) d^{2} v
$$


correspond to the graphs 3 and 4 ,

$$
T_{6}=\iint_{F} R(v, w) \partial_{v} \mathcal{D}_{z} G(z, v) G(v, v) \rho(v) d^{2} v
$$

while

$$
T_{7}=2 \iint_{F} \mathcal{D}_{w} \partial_{z} G(z, w) \partial_{z} G(z, v) G(v, v) \rho(v) d^{2} v
$$

corresponds to the graph 5 , and

$$
T_{8}=\left(2 \partial_{z} R(z, w)+R(z, w) \partial_{z}\right) \iint_{F} \mathcal{D}_{z} G(z, v) G(v, v) \rho(v) d^{2} v .
$$

Finally, the computation in Section 6.3 gives

$$
\begin{aligned}
P_{w}\left(E_{3}\right)(z, w) & =4 \iint_{F} \mathcal{D}_{w} \mathcal{D}_{\bar{u}} G(w, u) E_{3}(z, u) \rho(u)^{-1} d^{2} u \\
& =8 \iiint_{F} \int_{F} \mathcal{D}_{z} G(z, v) \mathcal{D}_{w} \mathcal{D}_{\bar{u}} G(w, u)\left(\partial_{u} G(u, v)\right)^{2} \rho(u)^{-1} \rho(v) d^{2} u d^{2} v \\
& =2 \iint_{F} \iint_{F} \mathcal{D}_{z} G(z, v) \mathcal{D}_{w} G(w, u) G(u, v)^{2} \rho(u) \rho(v) d^{2} u d^{2} v \\
& +2 \iint_{F} \mathcal{D}_{z} G(z, v)\left(\partial_{w} G(w, v)\right)^{2} \rho(v) d^{2} v \\
& +\iint_{F} \mathcal{D}_{z} G(z, v) R(v, w) \partial_{v} G(v, v) \rho(v) d^{2} v \\
& -\frac{1}{\pi} \iint_{F} \mathcal{D}_{z} G(z, v) \mathcal{D}_{w} G(w, v) \rho(v) d^{2} v \\
& =T_{9}+T_{10}+T_{11}+T_{12} .
\end{aligned}
$$

The first two terms $T_{9}$ and $T_{10}$ correspond to the graphs 6 and 7 . Using (5.31) we see that the terms $T_{6}, T_{11}$ and $T_{12}$ correspond to the remaining graph 8. Note that it is the term $T_{12}$ which is responsible for the regularization of the self-loop in Section 3.2.

Since $\partial^{2}=0$, we get from Theorem 6.1 that the kernel $\left.P_{z}\left(P_{w} E\right)\right)(z, w)$ is symmetric. Thus for the Fuchsian global coordinate on $X$,

$$
\begin{gathered}
\partial_{s}\langle\langle T(z) X\rangle\rangle_{1-\text { loop }}=-\pi P_{z}\left(P_{w}(E)\right)(z, w) \\
=-\frac{1}{\pi}\left(\langle\langle T(z) T(w) X\rangle\rangle_{1-\text { loop }}-\frac{1}{2} K(z, w)\right) \\
+\left(2 \partial_{z} R(z, w)+R(z, w) \partial_{z}\right)\langle\langle T(z) X\rangle\rangle_{1-\text { loop }}+T_{1}(z, w),
\end{gathered}
$$


where $T_{1}(z, w)=\pi P_{w}(E)(z, w)-\pi P_{z}\left(P_{w}(E)\right)(z, w)$ is a smooth quadratic differential in $z$ and $w$ which is holomorphic in $w$. It follows from the symmetry of $\langle\langle T(z) T(w) X\rangle\rangle_{1-\text { loop }}$ that it is a meromorphic quadratic differential in $z$ and $w$ with a fourth order pole at $z=w$.

To find $\partial_{s}\langle\langle T(z) X\rangle\rangle_{1-\text { loop }}$ for the Schottky global coordinate we observe that, according to (5.20), $\langle\langle T(z) X\rangle\rangle_{1-\text { loop }}-\frac{1}{12} \mathcal{S}\left(J^{-1}\right)(z)$ behaves as a quadratic differential under the change of global coordinates. Using formulas (5.37) and $\mathcal{S}\left(J^{-1}\right)(z)=0$, which are valid for the Fuchsian global coordinate on $X$, we obtain from (7.6) that for the Schottky global coordinate,

$$
\begin{gathered}
\partial_{s}\left(\langle\langle T(z) X\rangle\rangle_{1-\text { loop }}-\frac{1}{12} \mathcal{S}\left(J^{-1}\right)(z)\right) \\
=-\frac{1}{\pi}\left(\langle\langle T(z) T(w) X\rangle\rangle_{1-\text { loop }}-\frac{\pi}{6} \mathcal{D}_{z} \mathcal{D}_{w} G(z, w)\right) \\
+\left(2 \partial_{z} R(z, w)+R(z, w) \partial_{z}\right)\left(\langle\langle T(z) X\rangle\rangle_{1-\text { loop }}-\frac{1}{12} \mathcal{S}\left(J^{-1}\right)(z)\right)+T_{1}(z, w),
\end{gathered}
$$

Combining this formula with our computation of $\partial_{s} T_{c l}(z)$ in Section [7.1, we finally obtain

$$
\begin{aligned}
& \partial_{s}\langle\langle T(z) X\rangle\rangle_{1-\mathrm{loop}}=-\frac{1}{\pi}\left(\langle\langle T(z) T(w) X\rangle\rangle_{1-\text { loop }}-\frac{1}{2} K(z, w)\right) \\
& \quad+\left(2 \partial_{z} R(z, w)+R(z, w) \partial_{z}\right)\langle\langle T(z) X\rangle\rangle_{1-\text { loop }}+\tilde{T}_{1}(z, w),
\end{aligned}
$$

where $K(z, w)$ is again the kernel (5.36) for the Schottky group, and $\tilde{T}_{1}(z, w)$ is a smooth quadratic differential in $z$ and $w$ which is holomorphic in $w$. Using (6.7) we also get

$$
\begin{gathered}
\left(\partial_{s}-2 \partial_{z} R(z, w)-R(z, w) \partial_{z}\right) \log \langle X\rangle_{1-\text { loop }} \\
=\frac{1}{\pi^{2}}\left(\langle\langle T(z) T(w) X\rangle\rangle_{1-\text { loop }}-\frac{\pi}{6} \mathcal{D}_{z} \mathcal{D}_{w} G(z, w)\right)+T_{1}(z, w) .
\end{gathered}
$$

7.3. Higher loops. Similar to Section 6.3, define the map $p_{2}: \mathcal{G}_{z, w}^{(c)} \rightarrow \mathcal{G}_{z}^{(c)}$ by eliminating the vertex with label $w$ of valency 1 or 2 . We claim that for every $\Upsilon \in \mathcal{G}_{\geq 3}^{(c)}$ with more than one loop,

$$
\begin{gathered}
\sum_{\Upsilon^{\prime} \in p_{1}^{-1}(\Upsilon)}(-1)^{\left|V\left(\Upsilon^{\prime}\right)\right|+\varepsilon_{1}\left(\Upsilon^{\prime}\right)}\left(\partial_{s}-2 \partial_{z} R(z, w)-R(z, w) \partial_{z}\right) W_{\Upsilon^{\prime}}(X ; z) \\
=-2 \sum_{\Upsilon^{\prime \prime} \in\left(p_{2} \circ p_{1}\right)^{-1}(\Upsilon)}(-1)^{\left|V\left(\Upsilon^{\prime \prime}\right)\right|+\varepsilon_{1}\left(\Upsilon^{\prime \prime}\right)} W_{\Upsilon^{\prime \prime}}(X ; z, w) .
\end{gathered}
$$

This readily follows from the arguments in Sections 6.3 and 7.2 . Namely, when using the Leibniz rule we concentrate on the edge $e$ of $\Upsilon^{\prime}$ with neither of its endpoints being a labeled vertex of valency 1 or 2 , the result follows as in Section 6.3. When one of the endpoints of $e$ is a vertex with label 
$z$ of valency 1 or 2 , repeating arguments in Section 7.2 yields an extra contribution

$$
\left(2 \partial_{z} R(z, w)+R(z, w) \partial_{z}\right) W_{\Upsilon^{\prime}}(X ; z) .
$$

Putting everything together proves the theorem.

Remark 7.2. Equation (7.1) can be stated as $\left(P_{w} Q\right)(z, w)-\left(2 \partial_{z} R(z, w)+R(z, w) \partial_{z}\right)\langle\langle T(z) X\rangle\rangle=-\frac{1}{\pi}\left(\langle\langle T(z) T(w) X\rangle\rangle-\frac{c}{2} K(z, w)\right)$, with the kernel $Q(z, w)$ given by

$$
L_{\nu}\langle\langle T(z) X\rangle\rangle=\iint_{F} Q(z, w) \nu(w) d^{2} w .
$$

Using Remark [5.3, we can also state Theorem 7.1 as

$P_{w}\left(Q_{1}\right)(z, w)+\left(2 \partial_{z} \mathcal{R}(z, w)+\mathcal{R}(z, w) \partial_{z}\right)\langle\langle T(z) X\rangle\rangle=-\frac{1}{\pi}\left(\langle\langle T(z) T(w) X\rangle\rangle-\frac{c}{2} K(z, w)\right)$,

which clearly shows that $\langle\langle T(z) T(w) X\rangle\rangle$ is meromorphic in $z$ and $w$.

Corollary 7.3. For $\mu, \nu \in \Omega^{-1,1}(\Gamma)$,

$$
\begin{aligned}
& L_{\nu}\langle\langle T(z) X\rangle\rangle=-\frac{1}{\pi} \iint_{F}\left(\langle\langle T(z) T(w) X\rangle\rangle-\frac{c}{2} K(z, w)\right) \nu(w) d^{2} w, \\
& L_{\bar{\nu}}\langle\langle\bar{T}(\bar{z}) X\rangle\rangle=-\frac{1}{\pi} \iint_{F}\left(\langle\langle\bar{T}(\bar{z}) \bar{T}(\bar{w}) X\rangle\rangle-\frac{c}{2} K(\bar{z}, \bar{w})\right) \overline{\nu(w)} d^{2} w,
\end{aligned}
$$

where integrals are understood in the principal value sense.

Proof.

$$
\begin{aligned}
L_{\nu}\langle\langle T(z) X\rangle\rangle & =\iint_{F} Q(z, w) \nu(w) d^{2} w \\
& =\iint_{F}\left(Q_{1}(z, w)+\left(2 \partial_{z} \mathcal{R}(z, w)+\mathcal{R}(z, w) \partial_{z}\right)\langle\langle T(z) X\rangle\rangle\right) \nu(w) d^{2} w \\
& =\iint_{F}\left(P_{w} Q_{1}(z, w)+\left(2 \partial_{z} \mathcal{R}(z, w)+\mathcal{R}(z, w) \partial_{z}\right)\langle\langle T(z) X\rangle\rangle\right) \nu(w) d^{2} w
\end{aligned}
$$

Remark 7.4. It follows from Corollary 7.3 that for $\nu \in \Omega^{-1,1}(\Gamma)$

$$
\left.\iint_{F}\left(2 \partial_{z} R(z, w)+R(z, w) \partial_{z}\right)\langle\langle T(z) X\rangle\rangle\right) \nu(w) d^{2} w=0 .
$$


In fact, using (5.31) and orthogonality of $\mathcal{D}_{w} G$ to harmonic Beltrami differentials, we get for $q \in \Omega^{2,0}(\Gamma)$ and $\nu \in \Omega^{-1,1}(\Gamma)$

$$
\iint_{F}\left(2 \partial_{z} R(z, w)+R(z, w) \partial_{z}\right) q(z) \nu(w) d^{2} w=0 .
$$

We also have, in agreement with (7.4) and (7.9), that

$$
\iint_{F} \mathcal{D}_{z} \mathcal{D}_{w} G(z, w) \mu(w) d^{2} w=0 .
$$

Corollary 7.5. For $\mu, \nu \in \Omega^{-1,1}(\Gamma)$,

$$
\begin{aligned}
& L_{\mu} L_{\nu} \log \langle X\rangle=\frac{1}{\pi^{2}} \iint_{F} \iint_{F}\left(\langle\langle T(z) T(w) X\rangle\rangle-\frac{6}{\hbar} K(z, w)\right) \mu(z) \nu(w) d^{2} z d^{2} w, \\
& L_{\bar{\mu}} L_{\bar{\nu}} \log \langle X\rangle=\frac{1}{\pi^{2}} \iint_{F} \iint_{F}\left(\langle\langle\bar{T}(\bar{z}) \bar{T}(\bar{w}) X\rangle\rangle-\frac{6}{\hbar} K(\bar{z}, \bar{w})\right) \overline{\mu(z) \nu(w)} d^{2} z d^{2} w,
\end{aligned}
$$

where integrals are understood in the principal value sense.

Proof. Follows from Theorem 6.1, equation (4.11) and Corollary 7.3.

\section{TWO-POINT CORRELATION FUNCTION $-T \bar{T}$ EQUATION}

Here we compute the two point correlation function $\langle\langle T(z) \bar{T}(\bar{w}) X\rangle\rangle$ in all orders of the perturbation theory. Using notations in Section 4.3, we have the following result.

Theorem 8.1. On the Schottky space $\mathfrak{S}_{g}$,

$$
\bar{\partial}\langle\langle T(z) X\rangle\rangle=\frac{1}{\pi}\langle\langle T(z) \bar{T}(\bar{w}) X\rangle\rangle .
$$

The same statement holds for the Teichmüller space $\mathfrak{T}_{g}$.

Proof. We follow the proof of Theorem 7.1, using $L_{\bar{\nu}}$ instead of $L_{\nu}$.

8.1. Classical contribution. It follows from (4.12) and (5.27) that

$$
L_{\bar{\nu}} T_{c l}(z)=-\frac{1}{2 \hbar} \rho(z) \overline{\nu(z)}=-\frac{2}{\hbar} \iint_{F} \mathcal{D}_{z} \mathcal{D}_{\bar{w}} G(z, w) \overline{\nu(w)} d^{2} w
$$

Using identification in Section 4.3, we get

$$
\bar{\partial} T_{c l}(z)=\frac{2}{\hbar} \mathcal{D}_{z} \mathcal{D}_{\bar{w}} G(z, w)=\frac{1}{\pi}\langle\langle T(z) \bar{T}(\bar{w}) X\rangle\rangle_{c l} .
$$

This equation corresponds to the single tree graph in Fig. 3 with $w$ replaced by $\bar{w}$, and according to (4.10), it is equivalent to

$$
\bar{\partial} \partial \log \langle X\rangle_{c l}=\frac{i}{\pi \hbar} \omega_{W P}
$$


8.2. One-loop contribution. At the one loop level, using (5.27), parts (ii) and (iv) of Lemma 5.1, Corollary 5.5 and Lemma 5.6, we get

$$
\begin{aligned}
L_{\bar{\nu}}\langle\langle T(z) X\rangle\rangle_{1-\text { loop }}= & -\pi\left(L_{\bar{\nu}} H(z)+\iint_{F} L_{\bar{\nu}} \mathcal{D}_{z} G(z, u) G(u, u) \rho(u) d^{2} u\right. \\
& \left.+\iint_{F} \mathcal{D}_{z} G(z, u) L_{\bar{\nu}} G(u, u) \rho(u) d^{2} u+\frac{1}{2} \rho(z) \overline{\nu(z)} G(z, z)\right) \\
= & -\pi \iint_{F} \tilde{E}(z, w) \overline{\nu(w)} d^{2} w,
\end{aligned}
$$

where $\tilde{E}(z, w)=\tilde{E}_{1}(z, w)+\tilde{E}_{2}(z, w)+\tilde{E}_{3}(z, w)+\tilde{E}_{4}(z, w)+\tilde{E}_{5}(z, w)$, and

$$
\begin{aligned}
& \tilde{E}_{1}(z, w)=2\left(\partial_{z} \partial_{\bar{w}} G(z, w)\right)^{2}-\frac{1}{\pi} \mathcal{D}_{z} \mathcal{D}_{\bar{w}} G(z, w) \\
& \tilde{E}_{2}(z, w)=2 \iint_{F} \mathcal{D}_{z} \partial_{\bar{w}} G(z, w) \partial_{\bar{w}} G(w, u) G(u, u) \rho(u) d^{2} u \\
& \tilde{E}_{3}(z, w)=2 \iint_{F} \mathcal{D}_{z}(z, u)\left(\partial_{\bar{w}} G(w, u)\right)^{2} \rho(u) d^{2} u \\
& \tilde{E}_{4}(z, w)=-2 \mathcal{D}_{z} \mathcal{D}_{\bar{w}} G(z, w) \iint_{F} G(z, u) G(u, u) \rho(u) d^{2} u \\
& \tilde{E}_{5}(z, w)=2 \mathcal{D}_{z} \mathcal{D}_{\bar{w}} G(z, w) G(z, z)
\end{aligned}
$$

Here $\tilde{E}_{4}(z, w)$ and $\tilde{E}_{5}(z, w)$ are already anti-holomorphic quadratic differentials for $\Gamma$ in variable $w$, and we compute the corresponding orthogonal projections of $\tilde{E}_{1}(z, w), \tilde{E}_{2}(z, w)$ and $\tilde{E}_{3}(z, w)$ by using the Fuchsian global coordinate on $X \simeq \Gamma \backslash \mathbb{U}$. From the Stokes' theorem, property P3 and (5.31) it follows that

$$
\begin{aligned}
P_{\bar{w}}\left(\tilde{E}_{1}\right)= & 4 \iint_{F} \mathcal{D}_{\bar{w}} \mathcal{D}_{u} G(w, u)\left(2\left(\partial_{z} \partial_{\bar{u}} G(z, u)\right)^{2}-\frac{1}{\pi} \mathcal{D}_{z} \mathcal{D}_{\bar{u}} G(z, u)\right) \rho(u)^{-1} d^{2} u \\
= & -\iint_{F} \rho(u) R(\bar{u}, \bar{w}) \partial_{\bar{u}}\left(\partial_{z} G(z, u)\right)^{2} d^{2} u-\frac{1}{\pi} \mathcal{D}_{z} \mathcal{D}_{\bar{w}} G(z, w) \\
& -i \lim _{\varepsilon \rightarrow 0} \oint_{C_{\varepsilon}(w)} R(\bar{u}, \bar{w})\left(\partial_{z} \partial_{\bar{u}} G(z, u)\right)^{2} d \bar{u} \\
= & 2 \iint_{F} \mathcal{D}_{\bar{w}} G(w, u)\left(\partial_{z} G(z, u)\right)^{2} \rho(u) d^{2} u+2\left(\partial_{z} \partial_{\bar{w}} G(z, w)\right)^{2} \\
& -\frac{1}{\pi} \mathcal{D}_{z} \mathcal{D}_{\bar{w}} G(z, w)-\frac{i}{2} \lim _{\varepsilon \rightarrow 0} \oint_{C_{\varepsilon}(z)} R(\bar{u}, \bar{w})\left(\partial_{z} G(z, u)\right)^{2} \rho(u) d u \\
= & \tilde{T}_{1}+\tilde{T}_{2}+\tilde{T}_{3}+\tilde{T}_{4} .
\end{aligned}
$$


Terms $\tilde{T}_{1}$ and $\tilde{T}_{2}$ correspond to the contributions of graphs 1 and 2 , with $w$ replaced by $\bar{w}$, into $\langle\langle T(z) \bar{T}(\bar{w}) X\rangle\rangle_{1-\text { loop }}$, and

$$
\tilde{T}_{3}=-\frac{1}{\pi} \mathcal{D}_{z} \mathcal{D}_{\bar{w}} G(z, w) \text {. }
$$

To compute $\tilde{T}_{4}$, we use (5.9), (5.31) and

$$
\left(\partial_{z} G(z, u)\right)^{2}=\sum_{\gamma_{1} \neq \gamma_{2} \in \Gamma} \partial_{z} \mathcal{G}\left(z, \gamma_{1} u\right) \partial_{z} \mathcal{G}\left(z, \gamma_{2} u\right)+\sum_{\gamma \in \Gamma}\left(\partial_{z} \mathcal{G}(z, \gamma u)\right)^{2}
$$

to obtain

$$
\begin{aligned}
\tilde{T}_{4} & =\left.\rho(z) R(\bar{z}, \bar{w}) \sum_{\gamma \neq \mathrm{id} \in \Gamma} \partial_{z} \mathcal{G}(z, \gamma u)\right|_{u=z}-\frac{i}{2} \lim _{\varepsilon \rightarrow 0} \oint_{C_{\varepsilon}(z)} R(\bar{u}, \bar{w})\left(\partial_{z} \mathcal{G}(z, u)\right)^{2} \rho(u) d u \\
& =\frac{1}{2} \rho(z) R(\bar{z}, \bar{w}) \partial_{z}(G(z, z))+\frac{1}{\pi} \mathcal{D}_{z} \mathcal{D}_{\bar{w}} G(z, w) .
\end{aligned}
$$

To compute $P_{\bar{w}}\left(\tilde{E}_{2}\right)$ we observe that by the Stokes' theorem, property $\mathbf{P 3}$ and (5.31),

$$
\begin{gathered}
8 \iint_{F} \mathcal{D}_{\bar{w}} \mathcal{D}_{u} G(w, u) \mathcal{D}_{z} \partial_{\bar{u}} G(z, u) \partial_{\bar{u}} G(u, v) \rho(u)^{-1} d^{2} u \\
=-\iint_{F} R(\bar{u}, \bar{w}) \partial_{\bar{u}}\left(\mathcal{D}_{z} G(z, u) G(u, v)\right) \rho(u) d^{2} u \\
-i \lim _{\varepsilon \rightarrow 0} \oint_{C_{\varepsilon}} R(\bar{u}, \bar{w}) \mathcal{D}_{z} \partial_{\bar{u}} G(z, u) \partial_{\bar{u}} G(u, v) d \bar{u} \\
=2 \iint_{F} \mathcal{D}_{\bar{w}} G(w, u) \mathcal{D}_{z} G(z, u) G(u, v) \rho(u) d^{2} u+2 \mathcal{D}_{z} \partial_{\bar{w}} G(z, w) \partial_{\bar{w}} G(v, w) \\
+2 \partial_{z} \mathcal{D}_{\bar{w}} G(z, w) \partial_{z} G(z, v)+R(\bar{v}, \bar{w}) \mathcal{D}_{z} \partial_{\bar{v}} G(z, v)+2 \mathcal{D}_{z} \mathcal{D}_{\bar{w}} G(z, w) G(z, v),
\end{gathered}
$$

where $C_{\varepsilon}=C_{\varepsilon}(v) \cup C_{\varepsilon}(w) \cup C_{\varepsilon}(z)$. Thus we obtain

$$
\begin{aligned}
P_{\bar{w}}\left(\tilde{E}_{2}\right)(z, w) & =8 \iint_{F} \iint_{F} \mathcal{D}_{\bar{w}} \mathcal{D}_{u} G(w, u) \mathcal{D}_{z} \partial_{\bar{u}} G(z, u) \partial_{\bar{u}} G(u, v) G(v, v) \rho(u)^{-1} \rho(v) d^{2} u d^{2} v \\
& =\tilde{T}_{5}+\tilde{T}_{6}+\tilde{T}_{7}+\tilde{T}_{8}+\tilde{T}_{9}
\end{aligned}
$$

where

$$
\begin{aligned}
& \tilde{T}_{5}=2 \iint_{F} \iint_{F} \mathcal{D}_{\bar{w}} G(w, u) \mathcal{D}_{z} G(z, u) G(u, v) G(v, v) \rho(u) \rho(v) d^{2} u d^{2} v \\
& \tilde{T}_{6}=2 \iint_{F} \mathcal{D}_{z} \partial_{\bar{w}} G(z, w) \partial_{\bar{w}} G(w, v) G(v, v) \rho(v) d^{2} v \\
& \tilde{T}_{7}=2 \iint_{F} \partial_{z} \mathcal{D}_{\bar{w}} G(z, w) \partial_{z} G(z, v) G(v, v) \rho(v) d^{2} v
\end{aligned}
$$


correspond, respectively, to graphs 3,4 and 5 with $w$ replaced by $\bar{w}$, while

$$
\tilde{T}_{8}=\iint_{F} R(\bar{v}, \bar{w}) \mathcal{D}_{z} \partial_{\bar{v}} G(z, v) G(v, v) \rho(v) d^{2} v
$$

and

$$
\tilde{T}_{9}=-\tilde{E}_{4}(z, w) .
$$

Finally, as in Section 6.3, we obtain

$$
\begin{aligned}
P_{\bar{w}}\left(\tilde{E}_{3}\right)(z, w)= & 8 \iint_{F} \iint_{F} \mathcal{D}_{z} G(z, v) \mathcal{D}_{\bar{w}} \mathcal{D}_{u} G(w, u)\left(\partial_{\bar{u}} G(u, v)\right)^{2} \rho(u)^{-1} \rho(v) d^{2} u d^{2} v \\
= & 2 \iint_{F} \iint_{F} \mathcal{D}_{z} G(z, v) \mathcal{D}_{\bar{w}} G(w, u) G(u, v)^{2} \rho(u) \rho(v) d^{2} u d^{2} v \\
& +2 \iint_{F} \mathcal{D}_{z} G(z, v) \partial_{\bar{w}} G(w, v) \partial_{\bar{w}} G(w, v) \rho(v) d^{2} v \\
& +\iint_{F} \mathcal{D}_{z} G(z, v) R(\bar{v}, \bar{w}) \partial_{\bar{v}} G(v, v) \rho(v) d^{2} v \\
& -\frac{1}{\pi} \iint_{F} \mathcal{D}_{z} G(z, v) \mathcal{D}_{\bar{w}} G(w, v) \rho(v) d^{2} v \\
= & \tilde{T}_{10}+\tilde{T}_{11}+\tilde{T}_{12}+\tilde{T}_{13} .
\end{aligned}
$$

The first two terms, $\tilde{T}_{10}$ and $\tilde{T}_{11}$, correspond to graphs 6 and 7 . Using the Stokes' theorem and (5.31), we see that the sum $\tilde{T}_{3}+\tilde{T}_{4}+\tilde{E}_{5}+\tilde{T}_{8}+\tilde{T}_{12}+\tilde{T}_{13}$ corresponds to the remaining graph 8 . Thus we have proved

$$
\langle\langle T(z) \bar{T}(\bar{w}) X\rangle\rangle_{1-\text { loop }}=\pi^{2} P_{\bar{w}}(\tilde{E})(z, w),
$$

so that $\langle\langle T(z) \bar{T}(\bar{w}) X\rangle\rangle_{1-\text { loop }}$ is holomorphic in $z$ and anti-holomorphic in $w$. Hence

$$
\bar{\partial}\langle\langle T(z) X\rangle\rangle_{1-\text { loop }}=\frac{1}{\pi}\langle\langle T(z) \bar{T}(\bar{w}) X\rangle\rangle_{1-\text { loop }}
$$

8.3. Higher loops. As in Section 17.3, we claim that for every $\Upsilon \in \mathcal{G}_{\geq 3}^{(c)}$ with more than one loop,

$$
\begin{gathered}
\sum_{\Upsilon^{\prime} \in p_{1}^{-1}(\Upsilon)}(-1)^{\left|V\left(\Upsilon^{\prime}\right)\right|+\varepsilon_{1}\left(\Upsilon^{\prime}\right)} \bar{\partial} W_{\Upsilon^{\prime}}(X ; z) \\
=-2 \sum_{\Upsilon^{\prime \prime} \in\left(p_{2} \circ p_{1}\right)^{-1}(\Upsilon)}(-1)^{\left|V\left(\Upsilon^{\prime \prime}\right)\right|+\varepsilon_{1}\left(\Upsilon^{\prime \prime}\right)} W_{\Upsilon^{\prime \prime}}(X ; z, \bar{w}),
\end{gathered}
$$

where now $p_{2}: \mathcal{G}_{z, \bar{w}}^{(c)} \rightarrow \mathcal{G}_{z}^{(c)}$ is the map eliminating the labeled vertex $\bar{w}$ of valency 1 or 2 .

This readily follows from the arguments in Sections 6.3, 7.3 and 8.2. Since the only graph in $\mathcal{G}_{z}^{(c)}$ that contains an edge with both end points being a 
vertex of valency two is a one-loop tadpole graph, the computation is even simpler than is Section 7.3 .

It follows from Theorem 8.1 that

$$
L_{\bar{\nu}}\langle\langle T(z) X\rangle\rangle=-\frac{1}{\pi} \iint_{F}\langle\langle T(z) \bar{T}(\bar{w}) X\rangle\rangle \overline{\nu(w)} d^{2} w .
$$

Combining Theorems 6.1 and 8.1, we obtain

\section{Corollary 8.2.}

$$
\bar{\partial} \partial \log \langle X\rangle=-\frac{1}{\pi^{2}}\left(\langle\langle T(z) \bar{T}(\bar{w}) X\rangle\rangle-\frac{\pi}{6} \mathcal{D}_{z} \mathcal{D}_{\bar{w}} G(z, w)\right),
$$

or, equivalently,

$$
L_{\mu} L_{\bar{\nu}} \log \langle X\rangle=\frac{1}{\pi^{2}} \iint_{F} \iint_{F}\left(\langle\langle T(z) \bar{T}(\bar{w}) X\rangle\rangle-\frac{\pi}{6} \mathcal{D}_{z} \mathcal{D}_{\bar{w}} G(z, w)\right) \mu(z) \overline{\nu(w)} d^{2} z d^{2} w .
$$

\section{Conformal Ward identities and modular geometry}

According to Belavin, Polyakov and Zamolodchikov BPZ84, conformal symmetry of the two-dimensional quantum field theory on the Riemann sphere is expressed by the so-called conformal Ward identities for correlation functions with insertions of the stress-energy tensor. In particular, one-point Ward identities determine conformal dimensions of primary fields, while twopoint Ward identities describe the Virasoro algebra symmetry of a theory. BPZ conformal Ward identities were generalized to higher genus Riemann surfaces in [EO87].

As we have already mentioned, equations (6.3)-(6.4) and (7.1)-(77.2), (8.1) are one-point and two-point Ward identities for quantum Liouville theory on the higher genus Riemann surfaces. One-point Ward identities for the punctured Riemann sphere were discussed previously in [Tak94, Tak96a] ${ }^{9}$. Here we only observe that from (5.30) we obtain the following asymptotic for two-point correlation functions:

$$
\begin{aligned}
\langle\langle T(z) T(w) X\rangle\rangle= & \frac{c / 2}{(z-w)^{4}}+\frac{2}{(z-w)^{2}}\langle\langle T(z) X\rangle\rangle-\frac{1}{z-w} \partial_{z}\langle\langle T(z) X\rangle\rangle \\
& + \text { regular terms as } w \rightarrow z, \\
\langle\langle T(z) \bar{T}(\bar{w}) X\rangle\rangle= & \quad \text { regular terms as } w \rightarrow z, \\
\langle\langle\bar{T}(\bar{z}) \bar{T}(\bar{w}) X\rangle\rangle= & \frac{c / 2}{(\bar{z}-\bar{w})^{4}}+\frac{2}{(\bar{z}-\bar{w})^{2}}\langle\langle\bar{T}(\bar{z}) X\rangle\rangle-\frac{1}{\bar{z}-\bar{w}} \partial_{\bar{z}}\langle\langle\bar{T}(\bar{z}) X\rangle\rangle, \\
& + \text { regular terms as } w \rightarrow z,
\end{aligned}
$$

where $c=\frac{12}{\hbar}+1$. The leading terms in these equations are precisely BPZ Ward identities, where $c$ is the central charge of the theory.

\footnotetext{
${ }^{9}$ We plan to address this case in the forthcoming publication.
} 
As was pointed out in [Tak96a], equations (6.3)-(6.4) and (7.1)-(77.2), (8.1) also admit interpretation in terms of "modular geometry" of Friedan and Shenker. Actually, these equations give precise meaning to the discussion in [FS87, Section 3]. Namely, introducing $[10$

$$
\tilde{\mathcal{F}}=\mathcal{F}+\frac{1}{24 \pi} S_{c l}
$$

and using (4.11), we can rewrite (6.3) $-(6.4)$ as

$$
\begin{aligned}
& \partial \tilde{\mathcal{F}}_{X}=\frac{1}{\pi}\langle\langle T(z) X\rangle\rangle, \\
& \bar{\partial} \tilde{\mathcal{F}}_{X}=\frac{1}{\pi}\langle\langle\bar{T}(\bar{z}) X\rangle\rangle,
\end{aligned}
$$

where $\partial$ and $\bar{\partial}$ are $(1,0)$ and $(0,1)$ components of de Rham differential on $\mathfrak{S}_{g}$. Interpreting $e^{\tilde{\mathcal{F}}}$ as a Hermitian metrid11 in a trivial holomorphic line bundle $\mathfrak{S}_{g} \times \mathbb{C} \rightarrow \mathfrak{S}_{g}$, we see that $\frac{1}{\pi}\langle\langle T(z) X\rangle\rangle$ and $\frac{1}{\pi}\langle\langle\bar{T}(\bar{z}) X\rangle\rangle$ are $(1,0)$ and $(0,1)$ components of the corresponding canonical connection 12 in the unitary frame. It was proved by Zograf (see [Zog89, Theorem 3.1]) that the Hermitian metric $\exp \left\{\frac{1}{12 \pi} S_{c l}\right\}$ in $\mathfrak{S}_{g} \times \mathbb{C}$ descends to the Hermitian metric in the Hodge line bundle $\lambda_{H}$ over the moduli space $\mathfrak{M}_{g}$. Since $e^{\tilde{\mathcal{F}}}=e^{\frac{c}{24 \pi} S_{c l}} e^{\mathcal{F}_{0}}$, where $\mathcal{F}_{0}$ is a (formal) function on $\mathfrak{M}_{g}$, we see that the trivial holomorphic line bundle $\mathfrak{S}_{g} \times \mathbb{C} \rightarrow \mathfrak{S}_{g}$ with the Hermitian metric $e^{\tilde{\mathcal{F}}}$ descends to a "projective holomorphic line bundle" $\mathscr{E}_{c}=\lambda_{H}^{c / 2}$ over the moduli space $\mathfrak{M}_{g}$ (see [FS87] for the definition of a projective line bundle).

Correspondingly, Corollaries 7.3 and 8.2 can be interpreted as curvature computations for $\mathscr{E}_{c}$. Namely, denote by $\mathbf{1}$ the section of $\mathscr{E}_{C}$ whose pull-back to the trivial bundle over $\mathfrak{S}_{g}$ is a section identically equal to 1 , and by $\nabla_{\mu}$, $\mu \in \Omega^{-1,1}(\Gamma)$ - covariant derivative of the canonical connection. Using Corollary 7.3 we have

$$
\begin{aligned}
\nabla_{\mu} \nabla_{\nu} \mathbf{1}= & \frac{1}{\pi}\left(L_{\mu}+\frac{1}{\pi} \iint_{F}\langle\langle T(w) X\rangle\rangle \mu(w) d^{2} w\right) \iint_{F}\langle\langle T(z) X\rangle\rangle \nu(z) d^{2} z \\
= & -\frac{1}{\pi^{2}} \iint_{F} \iint_{F}\left(\langle\langle T(z) T(w) X\rangle\rangle-\frac{c}{2} K(z, w)\right) \mu(w) \nu(z) d^{2} w d^{2} z \\
& +\frac{1}{\pi^{2}} \iint_{X}\langle\langle T(z) X\rangle\rangle \nu(z) d^{2} z \iint_{X}\langle\langle T(w) X\rangle\rangle \mu(w) d^{2} w
\end{aligned}
$$

\footnotetext{
${ }^{10}$ It is interesting to interpret this finite one-loop redefinition of the free energy in invariant terms.

${ }^{11}$ Here we are tacitly assuming that $\tilde{\mathcal{F}}$ is a smooth function on $\mathfrak{S}_{g}$. Of course, it is only a formal function, so all geometric objects should be interpreted in a formal category.

12 The connection which is compatible with the Hermitian metric and complex structure in the line bundle.
} 
which is symmetric in $\nu$ and $\mu$, so that the $(2,0)$ component of the curvature tensor vanishes.

Similar statements hold for $(0,2)$ components. Finally, it follows from Corollary 8.2 that

$$
\begin{aligned}
\nabla_{\mu} \nabla_{\bar{\nu}} \mathbf{1} & =0 \\
\nabla_{\bar{\nu}} \nabla_{\mu} \mathbf{1} & =\frac{1}{\pi}\left(L_{\bar{\nu}} \iint_{F}\langle\langle T(z) X\rangle\rangle \mu(z) d^{2} z\right) \\
& =-\frac{1}{\pi^{2}} \iint_{F} \iint_{F}\langle\langle T(z) \bar{T}(\bar{w}) X\rangle\rangle \mu(z) \overline{\nu(w)} d^{2} z d^{2} w .
\end{aligned}
$$

Thus using the identification in Section 4.3 we see that the $(1,1)$ component of the curvature tensor is given by

$$
\frac{1}{\pi^{2}}\langle\langle T(z) \bar{T}(\bar{w}) X\rangle\rangle
$$

Remark 9.1. Since the Hodge line bundle $\lambda_{H}$ is positive, the projective line bundle $\mathscr{E}_{c}$ is also positive for $c>0$. Moreover, assuming that $\tilde{\mathcal{F}}$ is a function on $\mathfrak{S}_{g}$ given by the actual integral (3.1), the curvature form $\frac{1}{\pi^{2}}\langle\langle T(z) \bar{T}(\bar{w}) X\rangle\rangle$ of the canonical connection on $\mathscr{E}_{C}$ is a positive definite $(1,1)$ form on $\mathfrak{M}_{g}$. Indeed, denoting by $\mathscr{D}_{L} \varphi=e^{-\frac{1}{2 \pi \hbar} S(\varphi)} \mathscr{D} \varphi$ the corresponding measure on $\mathscr{C} \mathscr{M}(X)$ and using that

$$
\langle X\rangle=\int_{\mathscr{C} \mathscr{M}(X)} \mathscr{D}_{L} \varphi,
$$

we obtain for $\mu \in \Omega^{-1,1}(\Gamma)$,

$$
\begin{gathered}
\langle X\rangle^{2} \iint_{F} \iint_{F}\langle\langle T(z) \bar{T}(\bar{w}) X\rangle\rangle \mu(z) \overline{\mu(w)} d^{2} z d^{2} w \\
=\langle X\rangle \int_{\mathscr{C} \mathscr{M}(X)}\left|\iint_{F} T(\varphi)(z) \mu(z) d^{2} z\right|^{2} \mathscr{D}_{L} \varphi-\left|\int_{\mathscr{C} \mathscr{M}(X)} \iint_{F} T(\varphi)(z) \mu(z) d^{2} z \mathscr{D}_{L} \varphi\right|^{2},
\end{gathered}
$$

which is non-negative by Cauchy-Bunyakovskii inequality. In this way we get a Kähler metric $\omega$ on $\mathfrak{M}_{g}$, whose pull-back to $\mathfrak{S}_{g}$ has a Kähler potential $-\tilde{\mathcal{F}}$. The corresponding symplectic form $\omega$ is given by the following power series in $\hbar$,

$$
\omega=\frac{1}{2 \pi \hbar} \omega_{W P}+\sum_{n=0}^{\infty} \hbar^{n} \omega_{(n)} .
$$

It would be very interesting to give a geometric interpretation of these "quantum corrections" to the Weil-Petersson metric, and to understand the series (9.4) non-perturbatively. 


\section{Appendix A. Belavin-KNizhniK theOrem and the $T \bar{T}$ EQUATION}

Here we compare the one-loop $T \bar{T}$ equation in Corollary 8.2 with the special case of Belavin-Knizhnik theorem BK86 — a local index theorem for families of $\bar{\partial}$-operators on Riemann surfaces - a formula for the Chern form of Quillen's metric in the corresponding determinant line bundle over $\mathfrak{M}_{g}$. Using $\log \langle X\rangle_{1-\text { loop }}=-\frac{1}{2} \log Z(2)$, we get from Corollary 8.2 ,

$$
\begin{aligned}
L_{\mu} L_{\bar{\nu}} \log Z(2)= & -\frac{2}{\pi^{2}} \iint_{F} \iint_{F}\langle\langle T(z) \bar{T}(\bar{w}) X\rangle\rangle \mu(z) \bar{\nu}(w) d^{2} z d^{2} w \\
& +\frac{1}{12 \pi}(\mu, \nu),
\end{aligned}
$$

where $(\mu, \nu)$ stands for the inner product (4.1) in $\Omega^{-1,1}(\Gamma)$. On the other hand, using D'Hoker-Phong formula [DP86] det $\Delta_{2}=c_{g} Z(2)$, where $\Delta_{2}$ is the Laplace operator of the hyperbolic metric acting on quadratic differentials on $X$ and $c_{g}$ is a constant depending only on genus, the BelavinKnizhnik formula for the family of $\bar{\partial}$-operators acting on quadratic differentials can be written in the form

$$
L_{\mu} L_{\bar{\nu}} \log Z(2)-L_{\mu} L_{\bar{\nu}} \log \operatorname{det} N=\frac{13}{12 \pi}(\mu, \nu) .
$$

Here $N$ is a Gram matrix with respect to the inner product (4.4) of the bases of holomorphic quadratic differentials on the Riemann surfaces $X_{t}$, which depend holomorphically on $t \in \mathfrak{T}_{g}$ (see ZT87a for details and references).

We show how to obtain the Belavin-Knizhnik formula (A.2) from (A.1). First, using (4.8), [ZT87a, Lemma 1], formulas (2.8) in [ZT87a] and (1.3) in [TZ91, it is elementary to obtain

$$
\begin{aligned}
L_{\mu} L_{\bar{\nu}} \log \operatorname{det} N= & -\iint_{F} \iint_{F} P(z, z) G(z, w) \mu(w) \overline{\nu(w)} \rho(w) \rho(z)^{-1} d^{2} z d^{2} w \\
& -\iint_{F} \iint_{F} P(z, w) G(z, w) \mu(z) \overline{\nu(w)} d^{2} z d^{2} w
\end{aligned}
$$

where $P(z, w)=4 \mathcal{D}_{z} \mathcal{D}_{\bar{w}} G(z, w)$.

Remark A.1. Formula A.3 coincides with Wolpert's formula Wol86 for the Ricci tensor of the Weil-Petersson metric on $\mathfrak{T}_{g}$.

Now using the Fuchsian global coordinate on $X \simeq \Gamma \backslash \mathbb{U}$, we rewrite the first term in (A.3) as

$$
-\left.4 \iint_{F} \iint_{F} \sum_{\gamma \in \Gamma} \mathcal{D}_{z^{\prime}} \mathcal{D}_{\bar{z}} \mathcal{G}\left(z^{\prime}, \gamma z\right)\right|_{z^{\prime}=z} G(z, w) \mu(w) \overline{\nu(w)} \rho(w) \rho(z)^{-1} d^{2} z d^{2} w .
$$


Using (5.23) and (5.2), we obtain

$-\left.4 \iint_{F} \iint_{F} \mathcal{D}_{z^{\prime}} \mathcal{D}_{\bar{z}} \mathcal{G}\left(z^{\prime}, z\right)\right|_{z^{\prime}=z} G(z, w) \mu(w) \overline{\nu(w)} \rho(w) \rho(z)^{-1} d^{2} z d^{2} w=-\frac{3}{4 \pi}(\mu, \nu)$.

Using equations (4.2), (5.12), (5.23), property P3 and the Stokes' theorem, we can rewrite the remaining part of the first term in (A.3) as

$$
\begin{aligned}
& 4 \iint_{F}\left.\iint_{F} \sum_{\gamma \neq \mathrm{id} \in \Gamma} \partial_{z^{\prime}} \mathcal{D}_{\bar{z}} \mathcal{G}\left(z^{\prime}, \gamma z\right)\right|_{z^{\prime}=z} \partial_{z} G(z, w) \mu(w) \overline{\nu(w)} \rho(w) \rho(z)^{-1} d^{2} z d^{2} w \\
&=-\left.2 \iint_{F} \iint_{F} \sum_{\gamma \neq \mathrm{id} \in \Gamma} \partial_{z^{\prime}} \partial_{\bar{z}} \mathcal{G}\left(z^{\prime}, \gamma z\right)\right|_{z^{\prime}=z} G(z, w) \mu(w) \overline{\nu(w)} \rho(w) d^{2} z d^{2} w \\
&-\left.2 \iint_{F} \iint_{F} \sum_{\gamma \neq \mathrm{id} \in \Gamma} \partial_{\bar{z}} \mathcal{G}\left(z^{\prime}, \gamma z\right)\right|_{z^{\prime}=z} \partial_{z} G(z, w) \mu(w) \overline{\nu(w)} \rho(w) d^{2} z d^{2} w \\
&+\left.2 i \lim _{\varepsilon \rightarrow 0} \iint_{F} \oint_{C_{\varepsilon}(w)} \sum_{\gamma \neq \mathrm{id} \in \Gamma} \partial_{z^{\prime}} \partial_{\bar{z}} \mathcal{G}\left(z^{\prime}, \gamma z\right)\right|_{z^{\prime}=z} \partial_{z} G(z, w) \mu(w) \overline{\nu(w)} \rho(w) \rho(z)^{-1} d z d^{2} w \\
&=\left.\iint_{F} \iint_{F} \sum_{\gamma \neq \mathrm{id} \in \Gamma} \mathcal{G}\left(z^{\prime}, \gamma z\right)\right|_{z^{\prime}=z} G(z, w) \mu(w) \overline{\nu(w)} \rho(z) \rho(w) d^{2} z d^{2} w \\
& \quad+\left.2 \iint_{F} \sum_{\gamma \neq \mathrm{id} \in \Gamma} \partial_{z^{\prime}} \partial_{\bar{z}} \mathcal{G}\left(z^{\prime}, \gamma z\right)\right|_{z^{\prime}=z} \mu(z) \overline{\nu(z)} d^{2} z=J_{1}+J_{2} .
\end{aligned}
$$

Similarly, the second term in (A.3) can be rewritten as

$$
-4 \iint_{\mathbb{U}} \iint_{F} \mathcal{D}_{z} \mathcal{D}_{\bar{w}} \mathcal{G}(z, w) \sum_{\gamma \in \Gamma} \mathcal{G}(z, \gamma w) \mu(z) \overline{\nu(w)} d^{2} z d^{2} w
$$

To compute the contribution from $\gamma=\mathrm{id} \in \Gamma$, we use the identity

$$
\iint_{\mathbb{U}} \mathcal{D}_{z} \mathcal{D}_{\bar{u}} \mathcal{G}(z, u) \mathcal{G}(z, u) \mathcal{D}_{u} \mathcal{D}_{\bar{w}} \mathcal{G}(u, w) \rho(u)^{-1} d^{2} u=\frac{1}{12 \pi} \mathcal{D}_{z} \mathcal{D}_{\bar{w}} \mathcal{G}(z, w) .
$$

Indeed, denoting the integral by $B(z, w)$, we get from (5.5) that

$$
B(\sigma z, \sigma w) \sigma^{\prime}(z)^{2}{\overline{\sigma^{\prime}(w)}}^{2}=B(z, w)
$$

for all $\sigma \in \operatorname{PSL}(2, \mathbb{R})$, so it is sufficient to compute it at a fixed $z$. Using the unit disk $\mathbb{D}$ as a model for the hyperbolic plane (cf. with the proof of Lemma 5.6), it is easy to compute that $B(0, w)=\frac{1}{4 \pi^{2}}$, and the identity follows. Therefore,

$$
\begin{aligned}
& -4 \iint_{\mathbb{U}} \iint_{F} \mathcal{D}_{z} \mathcal{D}_{\bar{w}} \mathcal{G}(z, w) \mathcal{G}(z, w) \mu(z) \overline{\nu(w)} d^{2} z d^{2} w \\
= & -16 \iint_{F} \iint_{\mathbb{U}} \mu(z) B(z, w) \overline{\nu(w)} d^{2} z d^{2} w=-\frac{1}{3 \pi}(\mu, \nu) .
\end{aligned}
$$


Similarly, the remaining part of the second term in (A.3) can be rewritten as

$$
\begin{gathered}
4 \iint_{\mathbb{U}} \iint_{F} \partial_{z} \mathcal{D}_{\bar{w}} \mathcal{G}(z, w) \sum_{\gamma \neq \mathrm{id} \in \Gamma} \partial_{z} \mathcal{G}(z, \gamma w) \mu(z) \overline{\nu(w)} d^{2} z d^{2} w \\
-\iint_{F} \sum_{\gamma \neq \mathrm{id} \in \Gamma} \mathcal{G}(z, \gamma z) \mu(z) \overline{\nu(z)} \rho(z) d^{2} z \\
=-4 \iint_{\mathbb{U}} \iint_{F} \partial_{z} \partial_{\bar{w}} \mathcal{G}(z, w) \sum_{\gamma \neq \mathrm{id} \in \Gamma} \partial_{z} \partial_{\bar{w}} \mathcal{G}(z, \gamma w) \mu(z) \overline{\nu(w)} d^{2} z d^{2} w \\
+2 i \lim _{\varepsilon \rightarrow 0} \iint_{F} \oint_{C_{\varepsilon}(z)} \sum_{\gamma \neq \mathrm{id} \in \Gamma} \partial_{z} \partial_{\bar{w}} \mathcal{G}(z, \gamma w) \partial_{z} \mathcal{G}(z, w) \mu(z) \overline{\nu(w)} d w d^{2} z \\
-\iint_{F} \sum_{\gamma \neq \mathrm{id} \in \Gamma} \mathcal{G}(z, \gamma z) \mu(z) \overline{\nu(z)} \rho(z) d^{2} z \\
=J_{3}-J_{2}+J_{4} .
\end{gathered}
$$

Thus

$$
L_{\mu} L_{\bar{\nu}} \log \operatorname{det} N=-\frac{13}{12 \pi}(\mu, \nu)+J_{1}+J_{3}+J_{4}
$$

and using (5.15), (5.40) and (5.2), we finally obtain

$$
\begin{aligned}
L_{\mu} L_{\bar{\nu}} \log \operatorname{det} N= & \iint_{F} \iint_{F} G(z, z) G(z, w) \mu(w) \overline{\nu(w)} \rho(w) \rho(z) d^{2} z d^{2} w-\frac{1}{2 \pi}(\mu, \nu) \\
& -4 \iint_{F} \iint_{F}\left(\partial_{z} \partial_{\bar{w}} G(z, w)\right)^{2} \mu(z) \overline{\nu(w)} d^{2} z d^{2} w \\
& -\iint_{F} G(z, z) \mu(z) \overline{\nu(z)} \rho(z) d^{2} z
\end{aligned}
$$

Using this representation for $L_{\mu} L_{\bar{\nu}} \log \operatorname{det} N$, we get the Belavin-Knizhnik theorem (A.2) by carefully analyzing the contribution of each one-loop graph into (A.1). The corresponding computation is quite tedious and is based on the repeated use of the Stokes' theorem. In a sense, it reverses the computation in Section 8.2. We leave details to the interested reader. Instead, here we present a shortcut which uses (4.6) and Remark 6.3. Namely, from 
(4.6) and Lemma 5.6 we get

$$
\begin{aligned}
L_{\mu} L_{\bar{\nu}} \log Z(2)= & -2 L_{\bar{\nu}} \iint_{F}\left(H(z)+\frac{1}{12 \pi} \mathcal{S}\left(J^{-1}\right)(z)\right) \mu(z) d^{2} z \\
= & -4 \iint_{F} \iint_{F}\left(\partial_{z} \partial_{\bar{w}} G(z, w)\right)^{2} \mu(z) \overline{\nu(w)} d^{2} z d^{2} w+\frac{7}{12 \pi}(\mu, \nu) \\
& +4 \iint_{F} \iint_{F} H(z) \mathcal{D}_{\bar{z}} G(z, w) \mu(w) \overline{\nu(w)} \rho^{-1}(z) \rho(w) d^{2} z d^{2} w .
\end{aligned}
$$

Using (5.18), Stokes' theorem and (5.1), we can rewrite the last term as

$$
\begin{gathered}
-2 \iint_{F} \iint_{F} \partial_{z} G(z, z) \partial_{\bar{z}} G(z, w) \mu(w) \overline{\nu(w)} \rho^{-1}(z) \rho(w) d^{2} z d^{2} w \\
=\iint_{F} \iint_{F} G(z, z) G(z, w) \mu(w) \overline{\nu(w)} \rho(z) \rho(w) d^{2} z d^{2} w-\iint_{F} G(z, z) \mu(z) \overline{\nu(z)} \rho(z) d^{2} z .
\end{gathered}
$$

Combining this with the obtained expression for $L_{\mu} L_{\bar{\nu}} \log \operatorname{det} N$ gives (A.2).

Remark A.2. Thus the one-loop term in the $T \bar{T}$ equation can be viewed as another "packaging" of the local index theorem for families of $\bar{\partial}$-operators on Riemann surfaces. It would be interesting to find geometric interpretation of higher loop terms.

\section{Appendix B. The Stress-energy tensor And the ACtion FUNCTIONAL}

Let $z$ be a Schottky global coordinate on $X \simeq \Gamma \backslash \Omega$. For $\mu \in \mathcal{H}^{-1,1}(\Gamma)$ and sufficiently small $\varepsilon \in \mathbb{C}$, consider the holomorphic family $X^{\varepsilon \mu} \simeq \Gamma^{\varepsilon \mu} \backslash \Omega^{\varepsilon \mu}$, where $\Omega^{\varepsilon \mu}=f^{\varepsilon \mu}(\Omega)$ and $\Gamma^{\varepsilon \mu}=f^{\varepsilon \mu} \circ \Gamma \circ\left(f^{\varepsilon \mu}\right)^{-1}$. For given $\varphi \in \mathscr{C} \mathscr{M}(X)$, let $\varphi^{\varepsilon \mu} \in \mathscr{C} \mathscr{M}\left(X^{\varepsilon \mu}\right)$ be a smooth family defined by

$$
\varphi^{\varepsilon \mu} \circ f^{\varepsilon \mu}+\log \left|f_{z}^{\varepsilon \mu}\right|^{2}=\varphi .
$$

Lemma B.1. Let $S: \mathscr{C} \mathscr{M}(X) \rightarrow \mathbb{R}$ be the Liouville action functional defined by (2.4), and let $T(\varphi)=\varphi_{z z}-\frac{1}{2} \varphi_{z}^{2}$ be the corresponding $(2,0)$ component of the stress-energy tensor. We have

$$
\left.\frac{\partial}{\partial \varepsilon}\right|_{\varepsilon=0} S\left(\varphi^{\varepsilon \mu}\right)=2 \iint_{F} T(\varphi)(z) \mu(z) d^{2} z .
$$

Proof. It repeats verbatim the proof of Theorem 1 in [ZT87c]! Namely, condition (B.1), which replaces Ahlfors lemma used in [ZT87c], gives

$$
\begin{aligned}
& \dot{\varphi}_{z}+\varphi_{z z} \dot{f}=-\varphi_{z} \dot{f}_{z}-\dot{f}_{z z}, \\
& \dot{\varphi}_{\bar{z}}+\varphi_{z \bar{z}} \dot{f}=-\varphi_{z} \dot{f}_{\bar{z}}-\dot{f}_{z \bar{z}}
\end{aligned}
$$


where

$$
\dot{\varphi}=\left.\frac{\partial}{\partial \varepsilon} \varphi^{\varepsilon \mu}\right|_{\varepsilon=0},
$$

and the corresponding computation in ZT87c works line by line. The Gauss-Bonnet theorem, used in [ZT87c], is replaced by the equation

$$
\iint_{F \varepsilon \mu} e^{\varphi^{\varepsilon \mu}} d^{2} z=\iint_{F} e^{\varphi}\left(1-|\varepsilon \mu|^{2}\right) d^{2} z,
$$

which follows from (B.1).

Lemma B.1 gives a derivation of the stress-energy tensor from the Liouville action functional. We stress that the "transformation law" (B.1), and the form (2.4) of the action functional, both play a crucial role in this computation. The same statement holds for the Liouville action functional for the quasi-Fuchsian global coordinate, and the proof repeats verbatim the proof of Theorem 4.1 in [TT03a].

In conclusion, we present a heuristic derivation of the one-point conformal Ward identity, which clarifies corresponding arguments in [BPZ84. Namely, considering (B.1) as a "change of variables" in the functional integral

$$
\left\langle X^{\varepsilon \mu}\right\rangle=\int_{\mathscr{C} \mathscr{M}\left(X^{\varepsilon \mu}\right)} e^{-\frac{1}{2 \pi \hbar} S\left(\varphi^{\varepsilon \mu}\right)} \mathscr{D} \varphi^{\varepsilon \mu},
$$

and assuming that $\mathscr{D} \varphi^{\varepsilon \mu}=\mathscr{D} \varphi$, we obtain

$$
\begin{aligned}
L_{\mu}\langle X\rangle & =\left.\frac{\partial}{\partial \varepsilon}\right|_{\varepsilon=0}\left\langle X^{\varepsilon \mu}\right\rangle=\int_{\mathscr{C} \mathscr{M}(X)} L_{\mu} S(\varphi) e^{-\frac{1}{2 \pi \hbar} S(\varphi) \mathscr{D} \varphi} \\
& =\iint_{F}\langle T(z) X\rangle \mu(z) d^{2} z .
\end{aligned}
$$

Now every infinitesimally trivial Beltrami differential $\mu$ gives rise to a family $X^{\varepsilon \mu}$ conformally equivalent to $X$, so that $L_{\mu}\langle X\rangle=0$. This shows that $\langle T(z) X\rangle$ is a holomorphic quadratic differential for $\Gamma$.

As we have shown, there is a one-loop correction to this naive form of the Ward identity, which is due to the regularization of the divergent tadpole graph. Thus rigorous definition of the "integration measure" $\mathscr{D} \varphi$ (which, in particular, would make this and similar arguments work) is a non-trivial problem.

\section{REFERENCES}

[Ahl61] Lars V. Ahlfors, Some remarks on Teichmüller's space of Riemann surfaces, Ann. of Math. (2) $\mathbf{7 4}$ (1961), 171-191.

[Ahl87] _ Lectures on quasiconformal mappings, Wadsworth \& Brooks/Cole Advanced Books \& Software, Monterey, CA, 1987, With the assistance of Clifford J. Earle, Jr., Reprint of the 1966 original. 
[AT97] Ettore Aldrovandi and Leon A. Takhtajan, Generating functional in CFT and effective action for two-dimensional quantum gravity on higher genus Riemann surfaces, Comm. Math. Phys. 188 (1997), no. 1, 29-67.

[Ber70] Lipman Bers, Spaces of Kleinian groups, Several Complex Variables, I (Proc. Conf., Univ. of Maryland, College Park, Md., 1970), Springer, Berlin, 1970, pp. 9-34.

[BK86] A. A. Belavin and V. G. Knizhnik, Complex geometry and the theory of quantum strings, Zh. Èksper. Teoret. Fiz. 91 (1986), no. 2, 364-390 (Russian), English translation in Soviet Phys. JETP 64 (1986), 214-228.

[BPZ84] A. A. Belavin, A. M. Polyakov, and A. B. Zamolodchikov, Infinite conformal symmetry in two-dimensional quantum field theory, Nuclear Phys. B 241 (1984), no. $2,333-380$.

[DO94] H. Dorn and H. J. Otto, Two and three point functions in liouville theory, Nuclear Phys. B 429 (1994), no. 2, 375-388.

[DP86] Eric D'Hoker and D. H. Phong, On determinants of Laplacians on Riemann surfaces, Comm. Math. Phys. 104 (1986), no. 4, 537-545.

[EO87] Tohru Eguchi and Hirosi Ooguri, Conformal and current algebras on a general Riemann surface, Nuclear Phys. B 282 (1987), no. 2, 308-328.

[FS87] D. Friedan and S. Shenker, The analytic geometry of two-dimensional conformal field theory, Nuclear Phys. B 281 (1987), no. 3-4, 509-545.

[GJ87] James Glimm and Arthur Jaffe, Quantum physics, Springer-Verlag, New York, 1987.

[Kaz99] David Kazhdan, Introduction to QFT, Quantum fields and strings: a course for mathematicians, Vol. 1, 2 (Princeton, NJ, 1996/1997), Amer. Math. Soc., Providence, RI, 1999, pp. 377-418.

[Poi98] Henri Poincaré, Les fonctions fuchsiennes et l'équation $\Delta u=e^{u}$, J. Math. Pure Appl (5) 4 (1898), 137-230.

[Pol81] A. M. Polyakov, Quantum geometry of bosonic strings, Phys. Lett. B 103 (1981), no. 3, 207-210.

[Pol82] - unpublished, Lecture at Steklov Institute, Leningrad (1982).

[Ram90] Pierre Ramond, Field theory: a modern primer, Frontiers in Physics, vol. 74, Addison-Wesley Publishing Company Advanced Book Program, Redwood City, CA, 1990.

[Sar87] Peter Sarnak, Determinants of Laplacians, Comm. Math. Phys. 110 (1987), no. $1,113-120$.

[Sim74] Barry Simon, The $P(\phi)_{2}$ Euclidean (quantum) field theory, Princeton University Press, Princeton, N.J., 1974.

[Tak89] L. A. Takhtajan, Uniformization, local index theorem, and geometry of the moduli spaces of Riemann surfaces and vector bundles, Theta functions-Bowdoin 1987, Part 1 (Brunswick, ME, 1987), Proc. Sympos. Pure Math., vol. 49, Amer. Math. Soc., Providence, RI, 1989, pp. 581-596.

[Tak92] Leon Takhtajan, Semi-classical Liouville theory, complex geometry of moduli spaces, and uniformization of Riemann surfaces, New symmetry principles in quantum field theory (Cargèse, 1991), NATO Adv. Sci. Inst. Ser. B Phys., vol. 295, Plenum, New York, 1992, pp. 383-406.

[Tak93] Leon A. Takhtajan, Liouville theory: quantum geometry of Riemann surfaces, Modern Phys. Lett. A 8 (1993), no. 37, 3529-3535.

[Tak94] Liouville theory: Ward identities for generating functional and modular geometry, Modern Phys. Lett. A 9 (1994), no. 25, 2293-2299.

[Tak96a] L. A. Takhtajan, Topics in the quantum geometry of Riemann surfaces: twodimensional quantum gravity, Quantum groups and their applications in physics (Varenna, 1994), Proc. Internat. School Phys. Enrico Fermi, vol. 127, IOS, Amsterdam, 1996, pp. 541-579. 
[Tak96b] Leon A. Takhtajan, Equivalence of geometric $h<1 / 2$ and standard $c>25$ approaches to two-dimensional quantum gravity, Modern Phys. Lett. A 11 (1996), no. 2, 93-101.

[Tes01] J. Teschner, Liouville theory revisited, Class. Quant. Grav. 18 (2001), no. 23, R153-R222.

[TT03a] L. A. Takhtajan and L. P. Teo, Liouville action and Weil-Petersson metric on deformation spaces, global Kleinian reciprocity and holography, Comm. Math. Phys. 239 (2003), no. 1-2, 183-240.

[TT03b] Leon A. Takhtajan and Lee-Peng Teo, Weil-Petersson metric on the universal Teichmüller space I: Curvature properties and Chern forms, Preprint arXiv: math.CV/0312172 (2003).

[TZ91] L. A. Takhtajan and P. G. Zograf, A local index theorem for families of $\bar{\partial}$ operators on punctured Riemann surfaces and a new Kähler metric on their moduli spaces, Comm. Math. Phys. 137 (1991), no. 2, 399-426.

[Wit99] Edward Witten, Perturbative quantum field theory, Quantum fields and strings: a course for mathematicians, Vol. 1, 2 (Princeton, NJ, 1996/1997), Amer. Math. Soc., Providence, RI, 1999, pp. 419-473.

[Wol86] Scott A. Wolpert, Chern forms and the Riemann tensor for the moduli space of curves, Invent. Math. 85 (1986), no. 1, 119-145.

[Zog89] P. G. Zograf, Liouville action on moduli spaces and uniformization of degenerate Riemann surfaces, Algebra i Analiz 1 (1989), no. 4, 136-160 (Russian), English translation in Leningrad Math. J. 1 (1990), no. 4, 941-965.

[ZT87a] P. G. Zograf and L. A. Takhtadzhyan, A local index theorem for families of $\bar{\partial}$-operators on Riemann surfaces, Uspekhi Mat. Nauk 42 (1987), no. 6(258), 133-150 (Russian), English translation in Russian Math. Surveys 42 (1987), no. 6, 169-190.

[ZT87b] - On the Liouville equation, accessory parameters and the geometry of Teichmüller space for Riemann surfaces of genus 0 , Mat. Sb. (N.S.) 132(174) (1987), no. 2, 147-166 (Russian), English translation in Math. USSR Sb. 60 (1988), no. 1, 143-161.

[ZT87c] - On the uniformization of Riemann surfaces and on the Weil-Petersson metric on the Teichmüller and Schottky spaces, Mat. Sb. (N.S.) 132(174) (1987), no. 3, 304-321 (Russian), English translation in Math. USSR Sb. 60 (1988), no. 2, 297-313.

[ZZ96] A. B. Zamolodchikov and Al. B. Zamolodchikov, Structure constants and conformal bootstrap in liouville field theory, Nuclear Phys. B 477 (1996), no. 2, $577-605$.

Department of Mathematics, Stony Brook University, Stony Brook, Ny 11794-3651, USA

E-mail address: leontak@math.sunysb.edu

Faculty of Information Technology, Multimedia University, Jalan Multimedia, Cyberjaya, 63100, Selangor, Malaysia

E-mail address: lpteo@mmu.edu.my 